\title{
On the soft limit of open string disk amplitudes with massive states
}

\section{Bianchi and A.L. Guerrieri}

Dipartimento di Fisica, Università di Roma "Tor Vergata" and Sezione INFN di Roma II "Tor Vergata", Via della Ricerca Scientifica, 00133 Rome, Italy

E-mail: massimo.bianchi@roma2.infn.it, andrea.guerrieri@roma2.infn.it

ABSTRACT: We discuss the soft behaviour of open string amplitudes with gluons and massive states in any dimension. Notwithstanding non-minimal couplings of massive higher spin states to gluons, relying on OPE and factorization, we argue that the leading and subleading terms are universal and identical to the ones in Yang-Mills theories. In order to illustrate this, we compute some 4-point amplitudes on the disk involving gluons, massive states and, for the bosonic string, tachyons. For the superstring, we revisit the structure of the massive super-multiplets at the first massive level and rewrite the amplitudes in $D=4$ in the spinor helicity formalism, that we adapt to accommodate massive higher spin states. We also check the validity of a recently obtained formula relating open superstring amplitudes for mass-less states at tree-level to SYM amplitudes, by factorisation on two-particle massive poles. Finally we analyse the holomorphic soft limit of superstring amplitudes with one massive insertion.

KEywords: Superstrings and Heterotic Strings, Bosonic Strings

ARXiv EPRINT: 1505.05854 


\section{Contents}

1 Introduction and motivations 1

2 Open bosonic string amplitudes $\quad 2$

2.1 Vertex operators 3

2.2 Chan-Paton factors and twist symmetry 3

2.3 Four-point bosonic string amplitude 4

3 Open superstring amplitudes $\quad \mathbf{5}$

3.1 Vertex operators 5

3.2 Supersymmetry 6

3.3 Dimensional reduction to $D=4 \quad 7$

3.4 Four-point amplitudes (superstring) and spinor helicity basis $\quad 7$

3.4.1 Amplitude $\mathcal{A}_{A A A C}$ in $D \leq 10 \quad 9$

$\begin{array}{ll}3.4 .2 \mathcal{A}_{A A A C} \text { in } D=4 \text { helicity basis } & 10\end{array}$

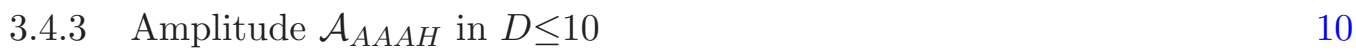

3.4.4 $\mathcal{A}_{A A A H}$ in $D=4$ helicity basis $\quad 11$

3.5 Higher-point open superstring amplitudes from SYM 11

$\begin{array}{lll}3.6 & 5 \text {-points in } D=4 \text { helicity basis } & 12\end{array}$

$\begin{array}{lll}3.7 & 6 \text {-points and higher point amplitudes } & 15\end{array}$

4 Soft limit $\quad \mathbf{1 5}$

$\begin{array}{lll}4.1 \text { General arguments } & 15\end{array}$

$\begin{array}{lll}4.2 & \text { Soft limit of } \mathcal{A}\left(A_{1}, T_{2}, T_{3}, T_{4}\right) & 18\end{array}$

$\begin{array}{lll}4.3 & \text { Soft limit of } \mathcal{A}\left(A_{1}, A_{2}, T_{3}, T_{4}\right) & 18\end{array}$

$\begin{array}{lll}4.4 & \text { Soft limit of } \mathcal{A}\left(T_{1}, T_{2}, A_{3}, H_{4}\right) & 19\end{array}$

4.5 Soft limit of $\mathcal{A}\left(A_{1}, A_{2}, A_{3}, C_{4}\right) \quad 20$

4.6 Soft limit of $\mathcal{A}\left(A_{1}, A_{2}, A_{3}, H_{4}\right) \quad 21$

5 Holomorphic soft limit $\quad 22$

$5.1 \mathcal{A}\left(A^{+}, A^{+}, A^{+}, C\right) \quad 22$

$5.2 \mathcal{A}\left(A^{-}, A^{+}, A^{-}, C\right) \quad 24$

$5.3 \mathcal{A}\left(A^{-}, A^{+}, A^{+}, H^{++}\right) \quad 25$

6 Conclusions $\quad 26$

$\begin{array}{ll}\text { A Open bosonic string 3-point amplitudes } & 27\end{array}$

$\begin{array}{ll}\text { B Open bosonic string four-point amplitudes } & 29\end{array}$

$\begin{array}{ll}\text { C Open superstring 3-point amplitudes } & 30\end{array}$ 
D Open superstring four-point amplitudes

D.1 $\mathcal{A}_{A A A C}$ amplitude $\quad 32$

D.2 $\mathcal{A}_{A A A H}$ amplitude $\quad 33$

D.3 $\mathcal{A}_{A A A C}$ in 4-dimensions $\quad 35$

D.4 $\mathcal{A}_{A A A H}$ in 4-dimensions $\quad 35$

\section{Introduction and motivations}

Recently the soft behaviour of scattering amplitudes has received renewed attention in connection with the extended BvBMS symmetry [1-6]. It has been long known that gauge theory and gravity amplitudes expose universal behaviours when one of the mass-less external momenta is 'soft' i.e. $k \rightarrow 0$ [7-9]. In both cases the leading behaviour is singular, i.e. goes as $\delta^{-1}$ if $k=\delta \hat{k}$ with $\hat{k}$ some fixed momentum [7]. The sub-leading terms can be derived from the leading ones and are largely fixed by gauge invariance. In particular, in gauge theories the sub-leading behaviour $\delta^{0}$ is universal, too. In gravity not only the sub-leading behaviour $\delta^{0}$ but also the next-to-subleading or sub-sub-leading behaviour $\delta^{+1}$ is universal [8-10].

The problem of what happens when loops or non-minimal higher derivative couplings are included was addressed in [11-15]. At the loop level IR divergences tend to spoil the analysis. Yet, in supersymmetric theories such as $\mathcal{N}=4 \mathrm{SYM}$, one can define loop integrands recursively and check that they expose the expected soft behaviour at all loops and for any choice of (massless) external legs. This may be viewed as a further constraint on (loop) amplitudes derived without resorting to standard perturbative methods (see e.g. [16] for a recent pedagogical review).

When non-minimal interactions are considered, the result depends on the specific choice [14]. In gauge theories, $F^{3}$ terms do not change the universal soft behaviour of minimal coupling, while $\phi F^{2}$ do modify even the leading term when $\phi$ is a massless scalar. Similarly, in gravity theories $R^{3}$ terms do not change the universal soft behaviour of minimal coupling, while $\phi R^{2}$ do modify even the leading term when $\phi$ is a massless scalar such as the dilaton.

These results are largely independent of the number of space-time dimensions and in particular apply to string theory in critical dimension and in lower dimensions [17-22]. One has to distinguish between open and closed strings and between bosonic, super and heterotic strings. In [14], the soft behaviour has been shown to be governed by the OPE of the vertex operators. As a result both open and closed superstring amplitudes with external massless states expose the expected soft behaviour, while closed bosonic string amplitudes don't, due to the tree-level non minimal coupling $\phi R^{2}$ with the dilaton. Open bosonic string amplitudes behave universally despite the presence already at tree level of the non-minimal $F^{3}$ correction to the standard Yang-Mills coupling and the coupling $T F^{2}$ to the tachyon. ${ }^{1}$ For the heterotic string at tree level, the soft behaviour of massless vector

\footnotetext{
${ }^{1}$ Couplings to gravitons and other closed string states appear at higher order in the string coupling $g_{s}$.
} 
bosons is universal, since the trilinear coupling is purely of Yang-Mills type, while the soft behaviour of the graviton is non-universal due to $\phi R^{2}$ coupling. ${ }^{2}$ No $R^{3}$ term however appears due to supersymmetry.

Aim of the present paper is to extend the analysis to open string amplitudes with massive external states (in the bosonic string case we will also consider tachyons as external states). Amplitudes with massive external states have not received much attention in the literature. See however [23-28] for recent work on the subject and [29-33] and the review [34] for mass-less amplitudes with emphasys on the exchange of massive higher spin states.

The analysis is interesting in two respects. On the one hand couplings of string states are generically non-minimal, although probably unique. On the other hand, gravity and gauge interactions emerge quite naturally in string theory and one would expect the soft behaviour of scattering amplitudes to expose some universality thanks to gauge invariance.

Plan of the paper is as follows. We start with open bosonic strings. After reviewing tri-linear couplings on the disk of tachyons, vector bosons and higher spin massive states, we compute some explicit 4-point amplitudes involving tachyons and massive states. We then consider open superstrings on the disk and perform a similar analysis in an arbitrary number of dimension $D \leq 10$. For convenience and for comparison with the existing literature we rewrite superstring amplitudes in $D=4$ in the spinor helicity formalism, that we adapt to accommodate massive higher spin states, after revisiting the structure of the massive super-multiplets. We also check the validity of a recently obtained formula relating open superstring amplitudes for mass-less states to SYM amplitudes at tree-level [35, 36], by factorizing 5-point amplitudes on the first massive pole and recovering our previous formulae. We explain how to generalise this procedure to an arbitrary number of massive external states. We then discuss the soft behaviour of open string amplitudes with gluons and massive states in any dimension and argue that the leading and sub-leading terms are universal and identical to the SYM case, relying on OPE and factorization. We then check this explicitly for the amplitudes, we previously computed. We also analyse the holomorphic soft limit of superstring amplitudes with one massive state and check consistency with our expectations. Finally we will draw our conclusions and identify interesting directions for future investigation. Various appendices contain technical details that are included for completeness.

\section{Open bosonic string amplitudes}

In order to check the soft behavior of four-point amplitudes on the disk in the open bosonic string, we summarize in appendix A all the possible tri-linear couplings involving the vertex operators up to the first massive level.

\footnotetext{
${ }^{2}$ M. B. would like I. Antoniadis to stressing the tree level origin of this term in the heterotic string, which only is generated at one-loop in 4-dim Type II theories with 16 supercharges, such as after compactification on $K 3 \times T^{2}$.
} 


\section{$2.1 \quad$ Vertex operators}

Our first goal is to compute scattering amplitudes with the insertion of vertex operators up to the first massive level. Up to normalization factors, the tachyon vertex operator is

$$
V_{T}=e^{i p X} \quad p^{2}=-m_{T}^{2}=\frac{1}{\alpha^{\prime}}
$$

the gluon vertex operator is

$$
V_{A}=a_{\mu} i \partial X^{\mu} e^{i k X} \quad k^{2}=-m_{A}^{2}=0 \quad a \cdot k=0
$$

and the first massive level vertex operator is

$$
\begin{aligned}
& V_{H}=H_{\mu \nu} i \partial X^{\mu} i \partial X^{\nu} e^{i p X} \\
& p^{2}=-m_{H}^{2}=-\frac{1}{\alpha^{\prime}} \\
& H_{\mu \nu}=H_{\nu \mu} \quad H_{\mu \nu} p^{\mu}=0 \quad \eta^{\mu \nu} H_{\mu \nu}=0 .
\end{aligned}
$$

While the choice of the tachyon vertex operator is essentially unique, the choice of the vertex operators for the massless gluon $A$ and for the massive state $H$ is not unique. It is always possible to add the null operator $c i k \partial X e^{i k X}=\delta_{B R S T} e^{i k X}$ to $c V_{A}$. For $H$ one can choose a linear combination of the operator $V_{B}=B_{\mu} i \partial^{2} X^{\mu} e^{i p X}(z)$ and a generic $\widetilde{H}(z)=$ $\widetilde{H}_{\mu \nu} i \partial X^{\mu} i \partial X^{\nu} e^{i p X}(z)$, with $\widetilde{H}$ an arbitrary two-index symmetric tensor. Nonetheless, due to BRST invariance, one has the freedom to fix the gauge in which $B_{\mu}=0$ and $H_{\mu \nu}$ is symmetric, traceless and traverse as in eq. (2.3).

\subsection{Chan-Paton factors and twist symmetry}

Although we will mostly consider 'color-ordered' amplitudes on the disk, we would like to review some relevant aspect of the group theory structure. Disk amplitudes are cyclically invariant and can be dressed with Chan-Paton factors [37]

$$
\mathcal{A}(1,2, \ldots n) \rightarrow \widehat{\mathcal{A}}(1,2, \ldots n)=\mathcal{A}(1,2, \ldots n) \operatorname{tr}\left(t^{1} \ldots t^{n}\right)
$$

where $t_{a}$ with $a=1, \ldots N^{2}$ are the generators of $\mathrm{U}(N) .^{3}$ In modern terms this corresponds to the fact that open strings carry multiplicities associated to the D-branes they end on. $\mathcal{A}(1,2, \ldots n)$ are called 'color-stripped' or 'color-ordered' amplitudes or simply sub-amplitudes. They enjoy such remarkable properties as [38]

- Cyclic symmetry: $\mathcal{A}(k, k+1, \ldots, n, 1,2, \ldots k-1)=\mathcal{A}(1,2, \ldots n)$

- Twist symmetry: $\mathcal{A}(n, n-1, \ldots, 2,1)=\prod_{i=1}^{n} \omega_{i} \mathcal{A}(1,2, \ldots, n-1, n)$

- Dual identity: $\mathcal{A}(1,2, \ldots n)+\mathcal{A}(2,1,3, \ldots n)+\ldots+\mathcal{A}(2,3, \ldots, n-1,1, n)=0$

where $\omega_{S}= \pm 1$ is the eigenvalue in the state $S$ of the 'twist' operator $\Omega$, that exchanges the two ends of the strings. In particular $\omega_{A}=-1$ while $\omega_{T, H}=+1$. In general $\omega_{S}=(-1)^{N_{S}}$

\footnotetext{
${ }^{3}$ We will not consider unoriented projections or symmetry breaking that may produce matter in bifundamental or (anti-)symmetric representations of the gauge group.
} 
where $N_{S}$ is the level of $S$. Pretty much as in gauge theory, complete amplitudes are obtained by summing over non-cyclical permutations of color-dressed amplitudes. At 3points one simply has

$$
\widehat{\mathcal{A}}(1,2,3)=\mathcal{A}(1,2,3) \operatorname{tr}\left(t^{1} t^{2} t^{3}\right)+\mathcal{A}(1,3,2) \operatorname{tr}\left(t^{1} t^{3} t^{2}\right) .
$$

For the amplitude with three tachyons $\mathcal{A}(1,3,2)=\mathcal{A}(3,2,1)=+\mathcal{A}(1,2,3)$, so that

$$
\widehat{\mathcal{A}}(1,2,3)=\mathcal{A}(1,2,3) \operatorname{tr}\left(t^{1} t^{2} t^{3}+t^{1} t^{3} t^{2}\right)=\mathcal{A}(1,2,3) d^{123},
$$

while for three vectors $\mathcal{A}(1,3,2)=\mathcal{A}(3,2,1)=-\mathcal{A}(1,2,3)$, so that

$$
\widehat{\mathcal{A}}(1,2,3)=\mathcal{A}(1,2,3) \operatorname{Tr}\left(t^{1} t^{2} t^{3}-t^{1} t^{3} t^{2}\right)=\mathcal{A}(1,2,3) f^{123} .
$$

In general one gets $d^{a b c}$ ('anomaly coefficients' or cubic Casimir) when $\prod_{i=1}^{3} \omega_{i}=+1$ and $f^{a b c}$ (structure constants) when $\prod_{i=1}^{3} \omega_{i}=-1$. In particular all couplings $\widehat{\mathcal{A}}(S, S, A) \sim f^{a b c}$ whichever the state $\mathcal{S}$. Moreover, at least for totally symmetric tensors in the first Regge trajectory, the dominant term at low energy is

$$
\mathcal{A}\left(S_{1}, S_{2}, A_{3}\right)=f_{123} S_{1}^{\mu_{1} \ldots \mu_{s}} S_{2, \mu_{1} \ldots \mu_{s}} a_{3} \cdot\left(p_{1}-p_{2}\right)+\ldots
$$

i.e. string theory tries to be as 'minimal' as it can! Yet there are higher derivative corrections to this, as we will see momentarily.

\subsection{Four-point bosonic string amplitude}

In this section we collect some open bosonic string amplitudes involving massless, massive and tachyonic states. Details of the computations can be found in appendix A. For simplicity we consider color-ordered amplitudes. Complete amplitudes arise after multiplying by the relevant Chan-Paton factors $\operatorname{tr}\left(t^{1} t^{2} t^{3} t^{4}\right)$ and summing over non-cyclic permutations. In fact, exploiting 'twist symmetry' i.e. $\Omega$ invariance, one can further reduce the sum to three terms (instead of six). For notational simplicity we will drop all adimensional constants (including powers of $g_{s}$ ) that are irrelevant for our analysis and the $\delta$-function of momentum conservation $(2 \pi)^{D} \delta^{D}\left(\sum_{i} p_{i}\right)$ will be understood. To help recognising the light-like momenta we will denote them by $k$ 's, while tachyonic and massive momenta will be denoted by $p$ 's. Starting with Veneziano amplitude (four tachyons)

$$
\mathcal{A}\left(T_{1}, T_{2}, T_{3}, T_{4}\right)=\frac{\Gamma\left(-1-\alpha^{\prime} s\right) \Gamma\left(-1-\alpha^{\prime} t\right)}{\Gamma\left(-2-\alpha^{\prime}(s+t)\right)}
$$

where $s=-\left(p_{1}+p_{2}\right)^{2}=-\left(p_{3}+p_{4}\right)^{2}, t=-\left(p_{2}+p_{3}\right)^{2}=-\left(p_{1}+p_{4}\right)^{2}, u=-\left(p_{1}+p_{3}\right)^{2}=$ $-\left(p_{2}+p_{4}\right)^{2}$, with $s+t+u=-4 / \alpha^{\prime}$, it is not difficult to compute the three-tachyons one-vector amplitude

$$
\mathcal{A}\left(A_{1}, T_{2}, T_{3}, T_{4}\right)=\frac{1}{\sqrt{2 \alpha^{\prime}}}\left(\frac{a_{1} p_{2}}{k_{1} p_{2}}-\frac{a_{1} p_{4}}{k_{1} p_{4}}\right) \frac{\Gamma\left(1+2 \alpha^{\prime} k_{1} p_{2}\right) \Gamma\left(1+2 \alpha^{\prime} k_{1} p_{4}\right)}{\Gamma\left(1-2 \alpha^{\prime} k_{1} p_{3}\right)},
$$

the two-tachyons two-vectors amplitude

$$
\mathcal{A}\left(A_{1}, A_{2}, T_{3}, T_{4}\right)=\left(\widetilde{a}_{1} \widetilde{a}_{2}+2 \alpha^{\prime} \widetilde{a}_{1} p_{3} \widetilde{a}_{2} p_{3}\right) \frac{\Gamma\left(1+2 \alpha^{\prime} k_{1} p_{4}\right) \Gamma\left(-1+2 \alpha^{\prime} k_{1} k_{2}\right)}{\Gamma\left(-2 \alpha^{\prime} k_{1} p_{3}\right)},
$$


where

$$
\widetilde{a}_{i}=a_{i}-\frac{a_{i} p_{4}}{k_{i} p_{4}} k_{i}, \quad i=1,2,
$$

satisfy $\widetilde{a}_{i} p_{4}=0$, and finally the two-tachyons-vector-tensor amplitude

$$
\begin{aligned}
\mathcal{A} & \left.T_{1}, T_{2}, A_{3}, H_{4}\right)=\frac{1}{\sqrt{2 \alpha^{\prime}}} \frac{\Gamma\left(-1+2 \alpha^{\prime} k_{3} p_{4}\right) \Gamma\left(1+2 \alpha^{\prime} p_{2} k_{3}\right)}{\Gamma\left(-2 \alpha^{\prime} p_{1} k_{3}\right)}\left[-2 a_{3} H p_{2}-2 a_{3} H k_{3} \frac{1+2 \alpha^{\prime} k_{3} p_{1}}{2-2 \alpha^{\prime} k_{3} p_{4}}\right. \\
& +2 \alpha^{\prime} a_{3} p_{4}\left(p_{2} H p_{2} \frac{1-2 \alpha^{\prime} k_{3} p_{4}}{2 \alpha^{\prime} k_{3} p_{1}}+k_{3} H k_{3} \frac{1+2 \alpha^{\prime} k_{3} p_{1}}{2-2 \alpha^{\prime} k_{3} p_{4}}+2 p_{2} H p_{3}\right) \\
& \left.-2 \alpha^{\prime} a_{3} p_{2}\left(\frac{k_{3} p_{4} p_{2} H p_{2}}{2 \alpha^{\prime} p_{2} k_{3} p_{1} k_{3}}\left(1-2 \alpha^{\prime} k_{3} p_{4}\right)-p_{3} H p_{3} \frac{1+2 \alpha^{\prime} p_{1} k_{3}}{2 \alpha^{\prime} p_{2} k_{3}}-2 p_{2} H k_{3} \frac{1-2 \alpha^{\prime} k_{3} p_{4}}{2 \alpha^{\prime} p_{2} k_{3}}\right)\right] .
\end{aligned}
$$

Later on we will check that they enjoy the expected behavior in the soft limit.

\section{Open superstring amplitudes}

\subsection{Vertex operators}

In this section we consider open superstring amplitudes involving gluons and massive states. At the first massive level, two independent string excitations appear: a symmetric, transverse and traceless tensor $H$ with $d_{H}=D(D-1) / 2-1$ degrees of freedom $\left(d_{H}=44\right.$ in $D=10)$ and a totally antisymmetric transverse tensor $C$ with $d_{C}=(D-1)(D-2)(D-3) / 6$ degrees of freedom $\left(d_{H}=84\right.$ in $\left.D=10\right)$. It is worth to notice that in $D=4$, the tensor $H$ corresponds to a massive spin 2 particle, while the $C$ corresponds to a massive pseudoscalar. Up to normalization factors, In the canonical $q=-1$ super-ghost picture their vertex operators are

$$
\begin{aligned}
& V_{A}^{(-1)}=e^{-\varphi} a \cdot \psi e^{i k X} \\
& k^{2}=0 \\
& k \cdot a=0 \\
& V_{H}^{(-1)}=H_{\mu \nu} e^{-\varphi} i \partial X^{\mu} \psi^{\nu} e^{i p X} \\
& \alpha^{\prime} p^{2}=-1 \\
& p_{\mu} H^{\mu \nu}=0 \\
& H_{\mu}^{\mu}=0 \\
& V_{C}^{(-1)}=C_{\mu \nu \rho} \psi^{\mu} \psi^{\nu} \psi^{\rho} e^{i p X} \\
& \alpha^{\prime} p^{2}=-1 \\
& p_{\mu} C^{\mu \nu \rho}=0 \text {. }
\end{aligned}
$$

For our purposes it is necessary to consider also vertex operators in the $q=0$ superghost picture

$$
\begin{aligned}
V_{A}^{(0)} & =(a \cdot i \partial X+k \cdot \psi a \cdot \psi) e^{i k X} ; \\
V_{H}^{(0)} & =H_{\mu \nu}\left[i \partial X^{\mu}\left(i \partial X^{\nu}+p \cdot \psi \psi^{\nu}\right]+\partial \psi \psi\right) e^{i p X} ; \\
V_{C}^{(0)} & =C_{\mu \nu \rho} e^{-\varphi}\left[i \partial X^{\mu}+p \cdot \psi \psi^{\mu}\right] \psi^{\rho} \psi^{\sigma} e^{i p X} .
\end{aligned}
$$

Higher spin massive states in the first Regge trajectory are described by vertex operators of the form

$$
V_{H_{s}}=H_{\mu_{1} \ldots \mu_{s}}\left[\prod_{i=1}^{s} i \partial X^{\mu_{i}}+p \psi \psi^{\mu_{1}} \prod_{i=2}^{s} i \partial X^{\mu_{i}}+(s-1) \partial \psi^{\mu_{1}} \psi^{\mu_{2}} \prod_{i=3}^{s} i \partial X^{\mu_{i}}\right] e^{i p X}
$$


with $\alpha^{\prime} p^{2}=(1-s)$ and $H$ totally symmetric, transverse and trace-less. Their tri-linear couplings to the vector bosons schematically read

$$
\mathcal{A}\left(A_{1}, A_{2}, H_{s}\right)=\left\langle c e^{-\varphi} V_{A}\left(z_{1}\right) c e^{-\varphi} V_{A}\left(z_{2}\right) c V_{H_{s}}\left(z_{3}\right)\right\rangle=\left(2 \alpha^{\prime}\right)^{s / 2}\left(f_{1} f_{2}\right)^{\mu_{1} \mu_{2}} H_{\mu_{1} \ldots \mu_{s}} \prod_{i=3}^{s} k_{12}^{\mu_{i}}
$$

In $D=4$ the above expressions drastically simplify if one resorts to the spinor helicity formalism and adapt it so as to encompass massive states.

\section{$3.2 \quad$ Supersymmetry}

Although we will only consider bosonic states in the NS sector of the open superstring, it is worth discussing the structure of the super-multiplet at the first massive level [39].

In addition to the NS states $H$ and $C$ we have two spin $3 / 2$ fermions of opposite chirality (in $D=10$ ) that combine to give a massive spin $3 / 2$ fermion. In the canonical $q=-1 / 2$ super-ghost picture their vertex operators read

$$
V_{\Psi}=\Psi_{\mu}^{\alpha} S_{\alpha} \partial X^{\mu} e^{-\varphi / 2} e^{i p X}
$$

and

$$
V_{\widetilde{\Psi}}=\widetilde{\Psi}_{\alpha}^{\mu} W_{\mu}^{\alpha} e^{-\varphi / 2} e^{i p X}
$$

where $S_{\alpha}$ is a spin field of conformal dimension $5 / 8$ in the 16 irrep of $\mathrm{SO}(1,9)$ and $W_{\mu}^{\alpha}=$ $: C^{\alpha} \psi^{\mu}$ : is an excited spin field of conformal dimension 13/8 in the $\mathbf{1 4 4}^{\prime}$ irrep of $\operatorname{SO}(1,9)$. BRST invariance implies transversality $p^{\mu} \Psi_{\mu}^{\alpha}=0=p_{\mu} \widetilde{\Psi}_{\alpha}^{\mu}$, $\Gamma$-traceleness $\Gamma_{\alpha \beta}^{\mu} \Psi_{\mu}^{\beta}=0=$ $\Gamma_{\mu}^{\alpha \beta} \widetilde{\Psi}_{\beta}^{\mu}$ as well as

$$
\Gamma_{\alpha \beta}^{\mu} p_{\mu} \Psi_{\nu}^{\beta}=i M \widetilde{\Psi}_{\nu, \alpha}, \quad \Gamma_{\mu}^{\alpha \beta} p^{\mu} \widetilde{\Psi}_{\nu, \beta}=i M \Psi_{\nu}^{\alpha}
$$

The $\mathcal{N}=(1,0)$ supersymmetry charge in $D=10$ is the gaugino vertex at zero momentum

$$
\mathcal{Q}_{\alpha}^{(-1 / 2)}=\int d z S_{\alpha} e^{-\varphi / 2}
$$

In the $q=+1 / 2$ super-ghost picture one has

$$
\mathcal{Q}_{\alpha}^{(+1 / 2)}=\int d z \Gamma_{\alpha \beta}^{\mu} C^{\beta} \partial X_{\mu} e^{+\varphi / 2}
$$

Acting with $\mathcal{Q}_{\alpha}^{(-1 / 2)}$ on $V_{\Psi}$ and $V_{\widetilde{\Psi}}$ one gets combinations of the NS vertex operators $V_{C}$ and $V_{H}$ as well as the 'auxiliary' vertices $V_{B}$ and $V_{E}$ in the canonical $q=-1$ picture, yielding very schematically ${ }^{4}$

$$
\delta H_{\mu \nu}=\varepsilon \Gamma_{(\mu} \Psi_{\nu)} \quad \delta C_{\mu \nu \rho}=\varepsilon \Gamma_{[\mu \nu} \widetilde{\Psi}_{\rho]} \quad \delta B_{\mu \nu}=\varepsilon \Gamma_{[\mu} \Psi_{\nu]} \quad \delta E_{\mu}=\varepsilon \widetilde{\Psi}_{\mu}
$$

Similarly acting with $\mathcal{Q}_{\alpha}^{(+1 / 2)}$ on the NS vertex operators $V_{C}$ and $V_{H}$ as well as on the 'auxiliary' vertices $V_{B}$ and $V_{E}$ yields very schematically ${ }^{5}$

$$
\delta \Psi_{\mu}=\varepsilon \Gamma_{\lambda}\left[p^{\lambda}\left(H_{\mu \nu}+B_{\mu \nu}\right) \Gamma^{\nu}+M \Gamma_{\mu} E^{\lambda}\right]+M C_{\mu \nu \rho} \Gamma^{\nu \rho} \varepsilon+\ldots
$$

\footnotetext{
${ }^{4}$ See [39] for the precise coefficients.

${ }^{5}$ See [39] for the precise coefficients.
} 
and

$$
\delta \widetilde{\Psi}_{\mu}=\varepsilon \Gamma^{\nu}\left[M\left(H_{\nu \mu}+B_{\nu \mu}\right)+p_{\nu} E_{\mu}\right]+\varepsilon \Gamma^{\nu} p_{\nu} C_{\mu \lambda \rho} \Gamma^{\lambda \rho}+\ldots
$$

\subsection{Dimensional reduction to $D=4$}

For obvious reasons we are particularly interested in the dimensional reduction to $D=4$. The massive $\mathcal{N}=(1,0)$ super-multiplet in $D=10$ at the first level yields a long multiplet of the $\mathcal{N}=4$ super-algebra

$$
\left\{H_{\mu \nu}, 8 \psi_{\mu}, 27 Z_{\mu}, 48 \chi, 42 \varphi\right\}
$$

comprising 128 bosonic and as many fermionic states. In order not to burden the notation $\mu, \nu, \ldots$ are now 4 -dim indices, while $i, j, \ldots$ denote the internal 6 dimensions. The origin of the bosonic fields is as follows

$$
\begin{aligned}
H_{\mu \nu} & \leftarrow H_{\mu \nu} \\
27 Z_{\mu} & \leftarrow 6 H_{\mu, i}, 15 C_{\mu, i j}, 6 C_{\mu \nu, i}
\end{aligned}
$$

since a massive vector in $D=4$ is equivalent to a massive anti-symmetric tensor, and

$$
42 \varphi \leftarrow 21 H_{i j}, 20 C_{i j k}, C_{\mu \nu \rho}
$$

It is perhaps not surprising that these be in one-to-one correspondence with the (bosonic) fields in the $\mathcal{N}=4$ super-current multiplet, upon dualizing the six massive $H_{\mu, i}$ into as many massive anti-symmetric tensors $\widetilde{H}_{\mu \nu, i}=\varepsilon_{\mu \nu \lambda \rho} p^{\lambda} H_{i}^{\rho} / M$. It is amusing to decompose this massive multiplet into massive multiplets of the $\mathcal{N}=1$ super-algebra

$$
\begin{aligned}
& \left\{H_{\mu \nu}, 8 \psi_{\mu}, 27 Z_{\mu}, 48 \chi, 42 \varphi\right\} \rightarrow \\
& \left\{H_{\mu \nu}, 2 \psi_{\mu}, Z_{\mu}\right\}+6\left\{\psi_{\mu}, 2 Z_{\mu}, \chi\right\}+14\left\{Z_{\mu}, 2 \chi, \varphi\right\}+14\{\chi, 2 \varphi\}
\end{aligned}
$$

In the case of a $\mathbf{Z}_{3}$ orbifold, whereby $x^{i} \rightarrow z^{I}, z_{\bar{I}}^{*}$ the multiplicities can be expressed in terms of dimensions of irreps of SU(3) i.e. $\quad 6 \rightarrow 3+3^{*}, \mathbf{1 4} \rightarrow \mathbf{8}+\mathbf{3}+\mathbf{3}^{*}$ and $14^{\prime} \rightarrow \mathbf{1}+\mathbf{1}+\mathbf{6}+\mathbf{6}^{*}$. Once again, it is not surprising that the multiplet content $\left\{H_{\mu \nu}, 2 \psi_{\mu}, Z_{\mu}\right\}$ be in one-to-one correspondence with the currents $\left\{T_{\mu \nu}, \Sigma_{\mu}, \bar{\Sigma}_{\mu}, J_{\mu}\right\}$ in the $\mathcal{N}=1$ super-current multiplet of Ferrara and Zumino [40].

For later purposes, note that $H_{\mu \nu}$, with $\eta^{\mu \nu} H_{\mu \nu}=0=p^{\mu} H_{\mu \nu}$ belongs in a spin2 supermultiplets with 8 bosonic and as many fermionic d.o.f. whose vector boson is $Z_{\mu}=\delta^{I \bar{J}} C_{\mu I \bar{J}}$, while $H_{\mu \nu}^{\prime}=H_{0}\left[\eta_{\mu \nu}+\alpha^{\prime} p_{\mu} p_{\nu}\right]$ with $H_{i j}=H_{0} \delta_{i j} / 2$ (so that $\left.\eta^{M N} H_{M N}=0\right)$ combine with $C_{0}=\varepsilon_{\mu \nu \rho \sigma} p^{\sigma} C^{\mu \nu \rho} / M$ in a chiral multiplet.

\subsection{Four-point amplitudes (superstring) and spinor helicity basis}

For simplicity, we will only consider amplitudes with a single massive external state: $\mathcal{A}\left(A_{1}, A_{2}, A_{3}, H_{4}\right)$ and $\mathcal{A}\left(A_{1}, A_{2}, A_{3}, C_{4}\right)$. Depending on the choice of incoming particles these correspond to production, annihilation or 3-body decay of the massive state. In view of this, it is useful to restrict to 4-dimensional momenta and polarisations and work in 
the helicity basis whereby null momenta are expressed in terms of on-shell Weyl spinors of opposite chirality

$$
k_{\alpha \dot{\alpha}}=k_{\mu} \sigma_{\alpha \dot{\alpha}}^{\mu}=u_{\alpha} \bar{u}_{\dot{\alpha}}
$$

and resort to the standard notation $u_{\alpha}(k) \rightarrow|k\rangle \bar{u}_{\dot{\alpha}}(k) \rightarrow[k \mid$, so much so that

$$
u\left(k_{i}\right) u\left(k_{j}\right)=-\langle i j\rangle, \quad \bar{u}\left(k_{i}\right) \bar{u}\left(k_{j}\right)=[i j] \quad \text { and } \quad 2 k_{i} \cdot k_{j}=\langle i j\rangle[i j]
$$

For real momenta $\bar{u}_{\dot{\alpha}}(k)=\left(u_{\alpha}(k)\right)^{*}$. Momentum conservation reads

$$
\sum_{i}|i\rangle\left[i\left|=0=\sum_{i}\right| i\right]\langle i| .
$$

Schoutens's identity entails $\langle 12\rangle\langle 34\rangle+\langle 13\rangle\langle 42\rangle+\langle 14\rangle\langle 23\rangle=0$ and a similarly for $\mid k]$ 's.

Positive and negative helicity polarisations can be expressed as

$$
a_{\alpha \dot{\alpha}}^{-}=a_{\mu}^{-} \sigma_{\alpha \dot{\alpha}}^{\mu}=\frac{u_{\alpha} \bar{v}_{\dot{\alpha}}}{\bar{u} \bar{v}} \quad \text { and } \quad a_{\alpha \dot{\alpha}}^{+}=a_{\mu}^{+} \sigma_{\alpha \dot{\alpha}}^{\mu}=\frac{v_{\alpha} \bar{u}_{\dot{\alpha}}}{u v},
$$

where $q_{\alpha \dot{\alpha}}=v_{\alpha} \bar{v}_{\dot{\alpha}}$ is an arbitrary light-like momentum that encodes the gauge freedom.

Also for massive particles it proves convenient to express their momenta and polarisations in terms of null momenta and Weyl spinors. Setting $p_{\alpha \dot{\alpha}}=k_{\alpha \dot{\alpha}}+q_{\alpha \dot{\alpha}}=u_{\alpha} \bar{u}_{\dot{\alpha}}+v_{\alpha} \bar{v}_{\dot{\alpha}}$ one has $p^{2}=2 k q=-m^{2}=u v \bar{u} \bar{v}$.

Helicity of a massive particle is not Lorentz invariant. For later purposes it proves convenient to explicitly identify the precise Lorentz transformations that map massive helicity states into one another. Let us choose the basis $\left\{u_{\alpha}, v_{\alpha}\right\}$ for Left-handed spinors with $u v=\langle u v\rangle \neq 0$ and $\left\{\bar{u}_{\dot{\alpha}}, \bar{v}_{\dot{\alpha}}\right\}$ for Right-handed spinors with $\bar{u} \bar{v}=[u v] \neq 0$. Dropping indices for simplicity, the Lorentz group $\mathrm{SL}(2, C) \times \overline{\mathrm{SL}(2, C)}$ act as

$$
L u=u^{\prime}=a u+b v \quad L v=v^{\prime}=c u+d v
$$

with $a, b, c, d \in \mathbf{C}$ such that $a d-b c=1$ and

$$
R \bar{u}=\bar{u}^{\prime}=\bar{a} \bar{u}+\bar{b} \bar{v} \quad R \bar{v}=\bar{v}^{\prime}=\bar{c} \bar{u}+\bar{d} \bar{v}
$$

It is easy to check that any symplectic product is invariant i.e. $\left\langle i^{\prime} j^{\prime}\right\rangle=\langle i j\rangle$ and $\left[i^{\prime} j^{\prime}\right]=[i j]$. The Lorentz transformations that leave the time-like momentum $p$ invariant form an $\mathrm{SO}(3)$ subgroup with

$$
a=e^{i \alpha} \cos \gamma, \quad b=e^{i \beta} \sin \gamma, \quad c=-e^{-i \beta} \sin \gamma, \quad d=e^{-i \alpha} \cos \gamma
$$

The $\mathrm{SO}(3)$ transformations

$$
L_{x}: \quad u^{\prime}=\frac{1}{\sqrt{2}}(u+v) \quad v^{\prime}=\frac{1}{\sqrt{2}}(-u+v)
$$

and

$$
L_{y}: \quad u^{\prime}=\frac{1}{\sqrt{2}}(u+i v) \quad v^{\prime}=\frac{1}{\sqrt{2}}(i u+v)
$$

with $R_{x / y}=L_{x / y}^{*}$ will prove particularly useful in the following. 
For a massive vector boson, with $p=u \bar{u}+v \bar{v}$ the three helicity states are $^{6}$

$$
w_{0}=u \bar{u}-v \bar{v} \quad w_{+}=u \bar{v} \quad w_{-}=v \bar{u}
$$

with $w_{0} \cdot w_{0}=4 m^{2}, w_{0} \cdot w_{ \pm}=0, w_{ \pm} \cdot w_{ \pm}=0, w_{ \pm} \cdot w_{\mp}=2 m^{2} .\left\{w_{0}, w_{+}, w_{-}\right\}$form a complete basis for transverse polarisations in that

$$
w_{0} \otimes w_{0}+w_{+} \otimes w_{-}+w_{-} \otimes w_{+}=2 m^{2} \eta+2 p \otimes p
$$

The complex circular polarisations $w_{ \pm}$can be combined into real ones

$$
w_{x}=u \bar{v}+v \bar{u} \quad w_{y}=i u \bar{v}-i v \bar{u}
$$

It is easy to check that $L_{x}$ maps $w_{x}$ into $w_{0}$ (up to a sign $L_{x} w_{x}=-w_{0}$ ) and vice versa $L_{x} w_{0}=+w_{x}$, leaving $w_{y}$ unaltered $L_{x} w_{y}=w_{y}$, while $L_{y}$ maps $w_{y}$ into $w_{0}\left(L_{y} w_{x}=w_{0}\right)$ and vice versa $L_{y} w_{0}=-w_{y}$, leaving $w_{x}$ unchanged $L_{y} w_{x}=w_{x}$.

For a massive tensor boson $(s=2)$, the five helicity states can be taken to be

$$
\begin{aligned}
H_{++} & =w_{+} \otimes w_{+} \quad H_{--}=w_{-} \otimes w_{-} & & H_{00}=w_{0} \otimes w_{0}-w_{+} \otimes w_{-}-w_{-} \otimes w_{+} \\
H_{+0} & =w_{+} \otimes w_{0}+w_{0} \otimes w_{+} & & H_{-0}=w_{-} \otimes w_{0}+w_{0} \otimes w_{-}
\end{aligned}
$$

Note that $w_{0} \otimes w_{0}+w_{+} \otimes w_{-}+w_{-} \otimes w_{+}=2 m^{2} \eta+2 p \otimes p$ is a scalar polarisation. As for the vector polarisations, the complex combinations $H_{ \pm \pm}$and $H_{ \pm 0}$ can be combined into real ones $H_{x x}-H_{y y}=H_{++}+H_{--}, H_{x y}=i H_{++}-i H_{--}, H_{x 0}=H_{+0}+H_{-0}, H_{y 0}=i H_{+0}-i H_{-0}$ ( $H_{00}$ is real). The transformation $L_{x}+L_{y}$ leaves $H_{++}+H_{--}$invariant, while $L_{x}-L_{y}$ maps $H_{++}+H_{--}$into $H_{00} . L_{x}$ maps $H_{++}-H_{--}$into $\left(H_{-0}-H_{+0}\right) / 2$ while $L_{y}$ maps $H_{++}-H_{--}$ into $-i\left(H_{+0}+H_{-0}\right) / 2$.

For spin $s$ totally symmetric tensors (as in the first Regge trajectory) one has $2 s+1$ helicity states, starting from the 'top' component $S_{++\ldots+}=(u \bar{v})^{s}=w_{+}^{s}$ to the 'bottom' $S_{--\ldots-}=(v \bar{u})^{s}=w_{-}^{s}$, passing through the middle components $S_{00 \ldots 0}=(u \bar{u}-v \bar{v})^{s}+\ldots=w_{0}^{s}+$ ... Applying combinations of the above $\mathrm{SO}(3)$ transformations (on the helicity spinors) one can map any amplitude, e.g. the one with the 'top' helicity component of a massive state, into any other. This applies independently for each external insertion.

\subsubsection{Amplitude $\mathcal{A}_{A A A C}$ in $D \leq 10$}

Let us start with $\mathcal{A}\left(A_{1}, A_{2}, A_{3}, C_{4}\right)$. With a judicious choice of super-ghost pictures and c-ghost insertions one has

$$
\begin{aligned}
& \mathcal{A}\left(A_{1}^{(-1)}, A_{2}^{(0)}, A_{3}^{(0)}, C_{4}^{(-1)}\right)=\lim _{\left(z_{1}, z_{2}, z_{4}\right) \rightarrow(\infty, 1,0)} \int_{0}^{1} d z_{3}\left\langle c e^{-\varphi} a_{1} \psi e^{i k_{1} X}\left(z_{1}\right)\right. \\
& \left.c\left(a_{2} i \partial X+k_{2} \psi a_{2} \psi\right) e^{i k_{2} X}\left(z_{2}\right)\left(a_{3} i \partial X+k_{3} \psi a_{3} \psi\right) e^{i k_{3} X}\left(z_{3}\right) c C_{4} \psi \psi \psi e^{i p_{4} X}\left(z_{4}\right)\right\rangle
\end{aligned}
$$

Following the steps detailed in appendix D.1, one finally gets

$$
\begin{aligned}
\mathcal{A}_{A A A C}= & \mathcal{B}(1,1)\left\{-a_{1} \cdot C_{4}: f_{2}\left[a_{3} \cdot k_{1}-\frac{u}{t} a_{3} \cdot k_{2}\right]-a_{1} \cdot C_{4}: f_{3}\left[\frac{u}{t} a_{2} \cdot k_{3}-\frac{u}{s} a_{2} \cdot k_{1}\right]+\frac{u}{s} a_{1} \cdot f_{2} \cdot C_{4}: f_{3}\right. \\
& \left.-a_{1} \cdot f_{3} \cdot C_{4}: f_{2}-2 \frac{u}{t} a_{1} \cdot \dot{C}_{4} \cdot \overline{f_{3}} \cdot \dot{f}_{2}\right\},
\end{aligned}
$$

\footnotetext{
${ }^{6}$ For a different basis of massive polarisations in 4-pt amplitudes, see [23, 24].
} 
where the contractions are performed in a self-explanatory fashion and

$$
\mathcal{B}(1,1)=\mathcal{B}\left(2 \alpha^{\prime} k_{3} p_{4}, 1+2 \alpha^{\prime} k_{2} k_{3}\right)=\frac{\Gamma\left(2 \alpha^{\prime} k_{3} p_{4}\right) \Gamma\left(1+2 \alpha^{\prime} k_{2} k_{3}\right)}{\Gamma\left(1+2 \alpha^{\prime} k_{3}\left(k_{2}+p_{4}\right)\right)} .
$$

Using $2 k_{1} k_{2}=-s=+2 k_{3} p_{4}-M^{2}, 2 k_{2} k_{3}=-t=+2 k_{1} p_{4}-M^{2}$ and $2 k_{3} k_{1}=-u=+2 k_{2} p_{4}-M^{2}$ i.e. $s+t+u=M^{2}=1 / \alpha^{\prime}$, one can check gauge invariance with respect to each of the three vector legs.

Expanding and shuffling all the terms in eq. (3.36), the amplitude $\mathcal{A}_{A A A C}$ can be written in a manifestly symmetric form under the exchange of the three vector boson legs

$$
\begin{aligned}
\mathcal{A}_{A A A C}= & 4 \alpha^{\prime} u \mathcal{B}(1,1)\left(C_{4}\left[a_{1} a_{2} a_{3}\right]+\sum_{i \neq 3} C_{4}\left[a_{1} a_{2} k_{i}\right] \frac{a_{3} k_{i}}{k_{3} k_{i}}+\sum_{i \neq 2} C_{4}\left[a_{3} a_{1} k_{i}\right] \frac{a_{2} k_{i}}{k_{2} k_{i}}+\right. \\
& \left.\sum_{i \neq 1} C_{4}\left[a_{2} a_{3} k_{i}\right] \frac{a_{1} k_{i}}{k_{1} k_{i}}+C_{4}\left[a_{1} k_{2} k_{3}\right] \frac{a_{2} a_{3}}{k_{2} k_{3}}+C_{4}\left[a_{2} k_{3} k_{1}\right] \frac{a_{3} a_{1}}{k_{3} k_{1}}+C_{4}\left[a_{3} k_{1} k_{2}\right] \frac{a_{1} a_{2}}{k_{1} k_{2}}\right),
\end{aligned}
$$

where $C[a b c]=C_{\mu \nu \rho} a^{\mu} b^{\nu} c^{\rho}$.

\subsection{2 $\mathcal{A}_{A A A C}$ in $D=4$ helicity basis}

In $D=4 C_{\mu \nu \rho}$ is equivalent to a (pseudo) scalar $C_{0}=\varepsilon^{\lambda \mu \nu \rho} p_{\lambda} C_{\mu \nu \rho} / 6 M$. In the helicity basis one has two independent color-ordered amplitudes $\mathcal{A}\left(1^{-} 2^{-} 3^{-} C_{0}\right)$ and $\mathcal{A}\left(1^{-} 2^{-} 3^{+} C_{0}\right)$ and their complex conjugates $\mathcal{A}\left(1^{+} 2^{+} 3^{+} C_{0}\right)$ and $\mathcal{A}\left(1^{+} 2^{+} 3^{-} C_{0}\right) .{ }^{7}$ The former reads

$$
\mathcal{A}\left(1^{-} 2^{-} 3^{-} C_{0}\right)=i \mathcal{B}(1,1) \frac{m_{C}\langle 13\rangle}{[12][23]} \text {. }
$$

The latter reads

$$
\mathcal{A}\left(1^{-} 2^{-} 3^{+} C_{0}\right)=i \mathcal{B}(1,1) \frac{[13]\langle 12\rangle^{3}}{m_{C}^{3}\langle 23\rangle} \text {. }
$$

\subsubsection{Amplitude $\mathcal{A}_{A A A H}$ in $D \leq 10$}

Let us now consider $\mathcal{A}_{A A A H}$. With a judicious choice of super-ghost pictures and c-ghost insertions one has

$$
\begin{gathered}
\mathcal{A}\left(A_{1}^{(0)}, A_{2}^{(-1)}, A_{3}^{(0)}, H_{4}^{(-1)}\right)=\lim _{\left(z_{1}, z_{2}, z_{4}\right) \rightarrow(\infty, 1,0)} \int_{0}^{1} d z_{3}\left\langle c\left(a_{1} i \partial X+k_{1} \psi a_{1} \psi\right) e^{i k_{1} X}\left(z_{1}\right)\right. \\
\left.c e^{-\varphi} a_{2} \psi e^{i k_{2} X}\left(z_{2}\right)\left(a_{3} i \partial X+k_{3} \psi a_{3} \psi\right) e^{i k_{3} X}\left(z_{3}\right) c \partial X \cdot H_{4} \cdot \psi e^{i p_{4} X}\left(z_{4}\right)\right\rangle .
\end{gathered}
$$

Following the steps detailed in appendix D.2, one finally finds

$$
\begin{aligned}
& \mathcal{A}_{A A A H}=\frac{1}{s t} \mathcal{B}\left(1-\alpha^{\prime} s, 1-\alpha^{\prime} t\right)\left\{a_{1} a_{3} s t\left[a_{2} H k_{1}\left(1-\alpha^{\prime} s\right)-a_{2} H k_{3}\left(1-\alpha^{\prime} t\right)\right]\right. \\
& +2 a_{1} H a_{2} s k_{1} f_{3} k_{2}-2 a_{3} H a_{2} t k_{3} f_{1} k_{2}+2 \alpha^{\prime}\left[s t\left(a_{3} k_{2} a_{1} k_{3} k_{3} H a_{2}-a_{1} k_{2} a_{3} k_{1} k_{1} H a_{2}\right)\right. \\
& \left.+a_{1} k_{2} a_{3} k_{2} u\left(k_{1} H a_{2} s-k_{3} H a_{2} t\right)\right]-2 a_{1} k_{2} a_{3} k_{1} a_{2} H k_{3}\left(1-\alpha^{\prime} t\right) t+2 a_{3} k_{2} a_{1} k_{3} a_{2} H k_{1}\left(1-\alpha^{\prime} s\right) s \\
& +a_{2} f_{3} H a_{1} u s-a_{2} f_{1} H a_{3} u t-2 \alpha^{\prime}\left(a_{3} k_{1} a_{2} f_{1} H k_{1}-a_{1} k_{3} a_{2} f_{3} H k_{3}\right) s t \\
& +2 \alpha^{\prime} a_{1} k_{2}\left(a_{2} f_{3} H k_{1} s-a_{2} f_{1} H k_{3} t\right) u-2 \alpha^{\prime} a_{3} k_{2}\left(a_{2} f_{1} H k_{3} t-a_{2} f_{1} H k_{1} s\right) u \\
& +2 a_{1} k_{3} a_{2} f_{3} H k_{1}\left(1-\alpha^{\prime} s\right) s-2 a_{3} k_{1} a_{2} f_{1} H k_{3}\left(1-\alpha^{\prime} t\right) t-2 \alpha^{\prime} s t\left(a_{2} f_{1} f_{3} H k_{1}-a_{2} f_{3} f_{1} H k_{3}\right) \\
& \left.-2 a_{2} f_{1} f_{3} H k_{3}\left(1-\alpha^{\prime} t\right) t+2 a_{2} f_{3} f_{1} H k_{1}\left(1-\alpha^{\prime} s\right) s\right\} .
\end{aligned}
$$

\footnotetext{
${ }^{7}$ Once again, details of the computations are relegated in appendix D.3.
} 
After laborious manipulations, this amplitude can be written in the compact symmetric form

$$
\begin{aligned}
\mathcal{A}_{A A A H}= & \frac{4}{s t} \mathcal{B}\left(1-\alpha^{\prime} s, 1-\alpha^{\prime} t\right)\left\{\left[2 \alpha^{\prime} f_{3} H f_{1} k_{3} f_{2} k_{1}+\left(1+2 \alpha^{\prime} k_{1} k_{3}\right) f_{3} H f_{1} f_{2}\right] k_{3} k_{1}\right. \\
& +\left[2 \alpha^{\prime} f_{1} H f_{2} k_{1} f_{3} k_{2}+\left(1+2 \alpha^{\prime} k_{1} k_{2}\right) f_{1} H f_{2} f_{3}\right] k_{1} k_{2} \\
& \left.+\left[2 \alpha^{\prime} f_{2} H f_{3} k_{2} f_{1} k_{3}+\left(1+2 \alpha^{\prime} k_{2} k_{3}\right) f_{2} H f_{3} f_{1}\right] k_{2} k_{3}\right\} .
\end{aligned}
$$

\subsection{4 $\mathcal{A}_{A A A H}$ in $D=4$ helicity basis}

Let us first consider the amplitudes involving the scalar component of $H$ and start with $\mathcal{A}\left(1^{+} 2^{+} 3^{+} H_{0}\right)=\mathcal{A}\left(1^{-} 2^{-} 3^{-} H_{0}\right)^{*}$. The amplitude can be written in the very compact form

$$
\mathcal{A}\left(1^{+} 2^{+} 3^{+} H_{0}\right)=\mathcal{B}(1,1) \frac{m_{H}[13]}{\langle 12\rangle\langle 23\rangle} .
$$

which is identical up to a phase to $\mathcal{A}\left(1^{+} 2^{+} 3^{+} C_{0}\right)$, for normalised states.

Consider a different choice for the helicity of the vectors in the amplitude with $H_{0}$ : $\mathcal{A}\left(1^{-} 2^{-} 3^{+} H_{0}\right)=\mathcal{A}\left(1^{+} 2^{+} 3^{-} H_{0}\right)^{*}$. The final result reads

$$
\mathcal{A}\left(1^{-} 2^{-} 3^{+} H_{0}\right)=\mathcal{B}(1,1) \frac{[13]\langle 12\rangle^{3}}{m_{H}^{3}\langle 23\rangle} .
$$

which is identical up to a phase to $\mathcal{A}\left(1^{-} 2^{-} 3^{+} C_{0}\right)$.

Consider now the the amplitude for the spin-2 tensor $H_{2}$ and three vector bosons $\mathcal{A}\left(1^{-} 2^{+} 3^{+} H_{2}^{h}\right)=\mathcal{A}\left(1^{+} 2^{-} 3^{-} H_{2}^{-h}\right)^{*}$. Setting $p=k_{4}+k_{5}$, the simplest amplitude to compute is the one for the state with polarisation $H^{++}=\langle 4|\langle 4|| 5]| 5]$ that reads

$$
\mathcal{A}\left(1^{-} 2^{+} 3^{+} H^{++}\right)=\mathcal{B}(1,1) \frac{\langle 14\rangle^{4}[13]}{m_{H}\langle 12\rangle\langle 23\rangle\langle 45\rangle^{2}} .
$$

The other amplitudes obtain in a straightforward way, after repeatedly applying $L_{x}$ and $L_{y}$ as outlined above. The final result can be compactly written as

$$
\begin{aligned}
& \sum_{h} c_{h} \mathcal{A}\left(1^{-} 2^{+} 3^{+} H^{h}\right)= \\
& \mathcal{B}(1,1) \frac{[13]\langle 14\rangle^{2}\langle 15\rangle^{2}}{m_{H}\langle 12\rangle\langle 23\rangle\langle 45\rangle^{2}}\left\{c_{++} \frac{\langle 14\rangle^{2}}{\langle 15\rangle^{2}}-4 c_{+0} \frac{\langle 14\rangle}{\langle 15\rangle}+6 c_{00}-4 c_{0-} \frac{\langle 15\rangle}{\langle 14\rangle}+c_{--} \frac{\langle 15\rangle^{2}}{\langle 14\rangle^{2}}\right\} .
\end{aligned}
$$

In the chosen orthogonal basis $\left|H_{++}\right|^{2}=\left|H_{--}\right|^{2}=4\left(k_{4} k_{5}\right)^{2}=m_{H}^{4}=\left(1 / \alpha^{\prime}\right)^{2},\left|H_{+0}\right|^{2}=\left|H_{0-}\right|^{2}=$ $16\left(k_{4} k_{5}\right)^{2}=4 m_{H}^{4}=\left(2 / \alpha^{\prime}\right)^{2}$ and $\left|H_{00}\right|^{2}=24\left(k_{4} k_{5}\right)^{2}=6 m_{H}^{4}=\left(\sqrt{6} / \alpha^{\prime}\right)^{2}$, so much so that $\hat{c}_{ \pm \pm}=$ $m_{H}^{2} c_{ \pm \pm}, \hat{c}_{ \pm 0}=2 m_{H}^{2} c_{ \pm 0}$ and $\hat{c}_{00}=\sqrt{6} m_{H}^{2} c_{00}$ for properly normalized polarization tensors.

\subsection{Higher-point open superstring amplitudes from SYM}

In $[35,36]$ Mafra, Schlotterer and Stieberger (MSS) have obtained a beautiful formula that allows one to express open superstring amplitudes for massless external states on the disk 
to SYM amplitudes at tree level. The formula is reminiscent of the KLT (Kawai, Lewellen, Tye) relations [41] and reads

$$
\mathcal{A}_{n}^{S T}(1,2, \ldots n)=\sum_{\sigma \in S_{n-3}} F\left(1\left[2_{\sigma} 3_{\sigma} \ldots n-2_{\sigma}\right] n-1, n\right) \mathcal{A}_{n}^{Y M}\left(1\left[2_{\sigma} 3_{\sigma} \ldots n-2_{\sigma}\right] n-1, n\right)
$$

with $z_{1}=0, z_{n-1}=1, z_{n}=\infty$ so that

$$
\begin{aligned}
& F(1[23 \ldots n-2] n-1, n)= \\
& \quad(-)^{n-3} \int_{z_{1}=0}^{1} d z_{2} \int_{z_{2}}^{1} d z_{3} \ldots \int_{z_{n-3}}^{z_{n-1}=1} d z_{n-2} \prod_{i<j} z_{i j}^{s_{i j}} \prod_{k=2}^{[n / 2]} \sum_{l=1}^{k-1} \frac{s_{l k}}{z_{l k}} \prod_{k=[n / 2]+1}^{n-1} \sum_{l=k+1}^{k-1} \frac{s_{k l}}{z_{k l}},
\end{aligned}
$$

where $s_{i j}=2 \alpha^{\prime} k_{i} k_{j}=-\alpha^{\prime} s_{i j}^{p h y s}$. The formula (3.49) follows from a tree-level CFT computation using the pure spinor formalism [35] and its soft limits and other properties were checked in [36]. A pure RNS derivation of eq. (3.49) has been given in [42], the proof is based on a revisited S-matrix approach [43]. We will here check that it is consistent with factorization on massive string states in two-particle channels i.e.

$$
\lim _{s_{12} \rightarrow-\alpha^{\prime} M_{H}^{2}}\left(s_{12}+\alpha^{\prime} M_{H}^{2}\right) \mathcal{A}_{n}\left(V_{1} V_{2} V_{3} \ldots V_{n}\right)=\sum_{H} \mathcal{A}_{3}\left(V_{1} V_{2} H\right) \mathcal{A}_{n-1}\left(H V_{3} V_{4} \ldots V_{n}\right),
$$

where $\mathcal{A}_{3}(V V H)$ is physical (decay rate, width) and can be computed for arbitrary states following the strategy outlined in appendix $\mathrm{C}$. This is nothing but $\operatorname{Res}\left[\mathcal{A}_{n}\left(V_{1} V_{2} V_{3} \ldots V_{n}\right)\right]$ for $s_{12}=-\alpha^{\prime} M_{H}^{2}$.

For simplicity will only consider mass-less 5-point amplitudes producing 4-point amplitudes with 3-massless and 1-massive state in $D=4$ and briefly mention how to generalize the procedure to an arbitrary number of mass-less and massive external states. In particular we give the relevant formula for mass-less 6-point amplitudes and sketch, at least in the MHV case, how to get the 4-massless and 1-massive at 5-points or the 2-massless and 2 -massive at 4-points.

\subsection{5-points in $D=4$ helicity basis}

The 5-point color-ordered amplitude for open superstring massless gluons reads [35, 36]

$$
\mathcal{A}_{5}(12345)=F(12345) \mathcal{A}_{5}^{Y M}(12345)+F(13245) \mathcal{A}_{5}^{Y M}(13245)
$$

where $F$ are multiple hyper-geometric functions

$$
F(1[23] 45)=s_{12} s_{34} \int_{0}^{1} d x \int_{x}^{1} d y x^{s_{12}-1} y^{s_{13}}(y-x)^{s_{23}}(1-x)^{s_{24}}(1-y)^{s_{34}-1},
$$

with $s_{i j}=2 \alpha^{\prime} k_{i} k_{j}$ and $F(13245)$ is obtained by exchanging 2 and $3,{ }^{8}$ i.e.

$$
F(1[32] 45)=s_{13} s_{24} \int_{0}^{1} d x \int_{x}^{1} d y x^{s_{12}} y^{s_{13}-1}(y-x)^{s_{23}}(1-x)^{s_{24}-1}(1-y)^{s_{34}} .
$$

\footnotetext{
${ }^{8}$ Notice that the notation for $F(12345)$ in eq. (3.52) might be confusing in that $F(13245)$ as a function of the momenta $k_{1}, k_{2}, k_{3}, k_{4}, k_{5}$ is not simply obtained from $F(12345)$ by exchanging $k_{2}$ and $k_{3}$.
} 
Since in $D=4$ any 5 -pt amplitude is either MHV or antiMHV with $\mathcal{A}_{5}^{\overline{M H V}}\left(1^{+} 2^{+} 3^{-} 4^{-} 5^{-}\right)=$ $\mathcal{A}_{5}^{M H V}\left(1^{-} 2^{-} 3^{+} 4^{+} 5^{+}\right)^{*}$, let us consider the MHV case for definiteness

$$
\mathcal{A}_{5}\left(1^{-} 2^{-} 3^{+} 4^{+} 5^{+}\right)=\frac{\langle 12\rangle^{3}}{\langle 23\rangle\langle 34\rangle\langle 45\rangle\langle 51\rangle} F(12345)+\frac{\langle 12\rangle^{4}}{\langle 13\rangle\langle 32\rangle\langle 24\rangle\langle 45\rangle\langle 51\rangle} F(13245)
$$

that can be written as

$$
\mathcal{A}_{5}\left(1^{-} 2^{-} 3^{+} 4^{+} 5^{+}\right)=\frac{\langle 12\rangle^{3}}{\langle 13\rangle\langle 23\rangle\langle 45\rangle\langle 51\rangle}\left[\frac{\langle 13\rangle}{\langle 34\rangle} F(12345)-\frac{\langle 12\rangle}{\langle 24\rangle} F(13245)\right] .
$$

MSS have checked the correct factorization on the massless poles [35, 36]. Here we will check consistency in the massive two-particle channel. To this end one has to take the residue at the pole $s_{12} \rightarrow-1$ respectively of $F(12345)$ and $F(13245)$. Starting from the expression

$$
F(12345)=s_{12} s_{34} \int_{0}^{1} d y \int_{0}^{y} d x x^{s_{12}-1}(1-x)^{s_{24}}(y-x)^{s_{23}}(1-y)^{s_{34}-1} y^{s_{13}}
$$

and making use of

$$
x^{s_{12}-1}=\frac{1}{s_{12}\left(s_{12}+1\right)} \frac{d^{2}}{d x^{2}} x^{s_{12}+1}
$$

in eq. (3.56) and integrating by parts, one finds

$$
F(12345)=s_{34} \int_{0}^{1} d y(1-y)^{s_{34}-1} y^{s_{13}} \int_{0}^{y} d x \frac{x^{s_{12}+1}}{s_{12}+1} \frac{d^{2}}{d x^{2}}\left[(1-x)^{s_{24}}(y-x)^{s_{23}}\right] .
$$

Now it is easy to take the residue and find

$$
\begin{aligned}
\operatorname{Res}_{s_{12}=-1} F(12345) & =\lim _{s_{12} \rightarrow-1}\left(s_{12}+1\right) F(12345) \\
& =s_{34} s_{24} \mathcal{B}\left(s_{13}+s_{23}+1, s_{34}\right)+s_{34} s_{23} \mathcal{B}\left(s_{13}+s_{23}, s_{34}\right) .
\end{aligned}
$$

Performing the same steps for

$$
F(13245)=s_{13} s_{24} \int_{0}^{1} d y \int_{0}^{y} d x x^{s_{12}}(1-x)^{s_{24}-1}(y-x)^{s_{23}}(1-y)^{s_{34}} y^{s_{13}-1},
$$

yields

$$
\underset{s_{12}=-1}{\operatorname{Res}} F(13245)=\lim _{s_{12} \rightarrow-1}\left(s_{12}+1\right) F(13245)=s_{13} s_{24} \mathcal{B}\left(s_{13}+s_{23}, s_{34}+1\right) .
$$

Finally the residue of the color-ordered string amplitude is

$$
\operatorname{Res}_{s_{12}=-1} \mathcal{A}_{5}^{S T}(12345)=s_{34} \mathcal{B}\left(s_{3 p}, s_{34}\right)\left\{\mathcal{A}_{5}^{Y M}(12345)\left[s_{23}-\frac{s_{24} s_{3 p}}{s_{35}}\right]-\mathcal{A}_{5}^{Y M}(13245) \frac{s_{13} s_{24}}{s_{35}}\right\},
$$

where $s_{3 p}=s_{13}+s_{23}=-s_{34}-s_{35}=\alpha^{\prime} t+\alpha^{\prime} u=1-\alpha^{\prime} s$, since $p=k_{1}+k_{2}$ and $2 k_{1} k_{2}=p^{2}=-M_{H / C}^{2}$. 
Using a mixed notation with both physical Mandelstam variables $(s, t, u)$ and $s_{i j}$ variables we obtain the following expression

$$
\begin{aligned}
& \quad \operatorname{Res}_{s_{12}}=-1 \\
& \quad=\mathcal{B}\left(1-\alpha^{\prime} s, 1-\alpha^{\prime} t\right)\left\{\mathcal{A}_{5}^{Y M}(12345)\left[s_{23} s_{35}+\left(s_{34}+s_{35}\right) s_{24}\right]-s_{13} s_{24} \mathcal{A}_{5}^{Y M}(13245)\right\} .
\end{aligned}
$$

One can check the factorization case by case, fixing the helicity of the external gluons. Before embarking in the computations, notice that only $\mathrm{SO}(6)$ singlet bosons can appear in the two-gluon channel. Following the dimensional reduction we previously revisited in some detail, one only has $H_{\mu \nu}, C_{\mu \nu \rho}=C_{0} \varepsilon_{\lambda \mu \nu \rho} p^{\lambda} / m_{C}$ and $\delta^{i j} H_{i j}=-\eta^{\mu \nu} H_{\mu \nu}=-3 H_{0}$, after decomposing $H_{\mu \nu}=H_{\mu \nu}^{t t}+H_{0}\left(\eta_{\mu \nu}+\alpha^{\prime} p_{\mu} p_{\nu}\right)$. Let us start with $\mathcal{A}\left(1^{-} 2^{-} 3^{+} 4^{+} 5^{+}\right)$. In this case we expect that only $C$ and $3 H_{0}=\eta^{\mu \nu} H_{\mu \nu}^{(4)}=\eta^{\mu \nu} H_{\mu \nu}^{(10)}=-\delta^{i j} H_{i j}^{(10)}$, with $H_{\mu \nu}^{(4)}=\eta_{\mu \nu}+\alpha^{\prime} p_{\mu} p_{\nu}$ the four-dimensional part of $H$, contribute. With this choice, eq. (3.62) becomes

$$
\begin{aligned}
& \operatorname{Res}_{s_{12}}=-1 \\
& =\mathcal{B}(1,1) \frac{\langle 12\rangle^{4}}{\langle 23\rangle\langle 45\rangle\langle 51\rangle}\left[\frac{\langle 23\rangle[23]\langle 35\rangle[35]+(\langle 34\rangle[34]+\langle 35\rangle[35])\langle 24\rangle[24]}{\langle 12\rangle\langle 34\rangle}-[13][24]\right] \\
& =\frac{\langle 12\rangle^{2}}{m_{H / C}} \mathcal{B}(1,1) \frac{m_{H / C}[35]}{\langle 45\rangle\langle 34\rangle}=\mathcal{A}_{3}\left(1^{-} 2^{-} H_{0}\right) \mathcal{A}_{4}\left(H_{0} 3^{+} 4^{+} 5^{+}\right)+\mathcal{A}_{3}\left(1^{-} 2^{-} C_{0}\right) \mathcal{A}_{4}\left(C_{0} 3^{+} 4^{+} 5^{+}\right),
\end{aligned}
$$

where $\mathcal{B}(1,1)=\mathcal{B}\left(1+2 \alpha^{\prime} k_{1} k_{2}, 1+2 \alpha^{\prime} k_{1} k_{3}\right)$. The result coincides with the one we previously derived using standard world-sheet techniques.

Consider now the amplitude $\mathcal{A}\left(1^{-} 2^{+} 3^{-} 4^{+} 5^{+}\right)$. As shown in table 1 in appendix D.4, if we take $k_{1 \alpha \dot{\alpha}}=u_{\alpha} \bar{u}_{\dot{\alpha}}$ and $k_{2 \beta \dot{\beta}}=v_{\beta} \bar{v}_{\dot{\beta}}$, with $k_{1}, k_{2}$ such that $2 k_{1} \cdot k_{2}=p^{2}=-1 / \alpha^{\prime}$, we find that only the spin-2 polarization $v_{\alpha} v_{\beta} \bar{u}_{\dot{\alpha}} \bar{u}_{\dot{\beta}}$ contributes at the massive pole. With this choice, we have

$$
\begin{aligned}
& \operatorname{Res}_{s_{12}=-1} \mathcal{A}_{5}^{S T}\left(1^{-} 2^{+} 3^{-} 4^{+} 5^{+}\right) \\
& =\mathcal{B}(1,1) \frac{\langle 13\rangle^{4}\{\langle 23\rangle[23]\langle 35\rangle[35]+(\langle 34\rangle[34]+\langle 35\rangle[35])\langle 24\rangle[24]+[13][24]\langle 12\rangle\langle 34\rangle\}}{\langle 12\rangle\langle 23\rangle\langle 34\rangle\langle 45\rangle\langle 51\rangle} \\
& =m_{H} \mathcal{B}(1,1) \frac{\langle 13\rangle^{4}[35]}{m_{H}\langle 12\rangle^{2}\langle 34\rangle\langle 45\rangle}=\sum_{h} \mathcal{A}_{3}\left(1^{-} 2^{+} H^{h}\right) \mathcal{A}_{4}\left(H^{-h} 3^{-} 4^{+} 5^{+}\right) .
\end{aligned}
$$

where only $\left.\left.H^{++}=|1\rangle|1\rangle \mid 2\right] \mid 2\right]$ contributes since $\mathcal{A}_{3}\left(1^{-} 2^{+} H^{++}\right)=m_{H}=-2 \sqrt{\alpha^{\prime}} k_{1} k_{2}$, while for the remaining helicity states $\mathcal{A}_{3}\left(1^{-} 2^{+} H^{h \neq++}\right)=0$. The result coincides with the one we previously derived using standard world-sheet techniques.

The last case is the amplitude $\mathcal{A}\left(1^{+} 2^{+} 3^{-} 4^{-} 5^{+}\right)$in which, as for the first case, only $H_{0}$ and $C_{0}$ get exchanged in the $s_{12}$ channel.

$$
\begin{aligned}
& \operatorname{Res}_{s_{12}=-1} \mathcal{A}_{5}^{S T}\left(1^{+} 2^{+} 3^{-} 4^{-} 5^{+}\right) \\
& =\mathcal{B}(1,1) \frac{\langle 34\rangle^{3}}{\langle 12\rangle\langle 23\rangle\langle 45\rangle\langle 51\rangle}(\langle 23\rangle[23]\langle 35\rangle[35]+(\langle 34\rangle[34]+\langle 35\rangle[35])\langle 24\rangle[24]+[13][24]\langle 12\rangle\langle 34\rangle) \\
& =\frac{[12]^{2}}{m_{H / C}} \mathcal{B}(1,1) \frac{\langle 34\rangle^{3}[35]}{m_{H / C}^{3}\langle 45\rangle}=\mathcal{A}_{3}\left(1^{+} 2^{+} H / C\right) \mathcal{A}_{4}\left(H / C, 3^{-} 4^{-} 5^{+}\right) .
\end{aligned}
$$

The result coincides with the one previously derived using standard world-sheet techniques. 


\subsection{6-points and higher point amplitudes}

Open-string amplitudes with more than one massive insertion look somewhat cumbersome and not very illuminating in $D=10$. In $D=4$, in the spinor helicity basis, formulae look more compact. A possible strategy for systematic computations is to derive amplitudes for massive states by multiple factorization of amplitudes for massless states on massive poles in two-particle channels. For open superstrings in turn one can rely on the MSS formula $[35,36]$, relating string amplitudes to SYM amplitudes, whose validity we have given further support earlier on.

For instance at 6-points, there are six terms in the MSS formula, corresponding to the permutations of [234] i.e.

$$
\begin{aligned}
\mathcal{A}_{6}^{S T}(123456)= & F(1[234] 56) \mathcal{A}_{6}^{Y M}(1[234] 56)+F(1[342] 56) \mathcal{A}_{6}^{Y M}(1[342] 56) \\
& +F(1[423] 56) \mathcal{A}_{6}^{Y M}(1[423] 56)+F(1[324] 56) \mathcal{A}_{6}^{Y M}(1[324] 56) \\
& +F(1[432] 56) \mathcal{A}_{6}^{Y M}(1[432] 56)+F(1[243] 56) \mathcal{A}_{6}^{Y M}(1[243] 56)
\end{aligned}
$$

Differently from the 4- and 5-point cases where only MHV (or anti-MHV) amplitudes are non-zero, at 6-point one has a NMHV amplitude $\mathcal{A}_{6}^{N M H V}(---+++)$ that even in SYM has a lengthy expression if compared to Parke-Taylor formula [38]. Focussing on MHV amplitudes $\mathcal{A}_{6}^{M H V}(--++++)=\langle 12\rangle^{3} /\langle 23\rangle\langle 34\rangle \ldots\langle 61\rangle$ one can still compute 5point amplitudes with one massive insertion with almost no effort and 4-point amplitudes with two massive insertions with little more effort.

For an arbitrary number of external massless legs $n$ a priori one has $\mathrm{N}^{k} \mathrm{MHV}$ amplitudes with $k=0, \ldots[n / 2]-2$. These, and susy related ones, are needed to compute amplitudes for generic massive states by factorization. Summarizing one can start with $\mathcal{A}_{n+2 m}^{S Y M, k}$, then derive $\mathcal{A}_{n+2 m}^{S T, k}$ and finally obtain $\mathcal{A}_{n, m}^{S T, k}$ by factorization on the assigned two-particle massive poles. Notice that the initial helicity configuration should be chosen compatibly with the choice of massive states, i.e. at the first level $H^{0} / C^{0}$ couple to gluons with the same helicity while $\mathrm{H}_{2}$ couples to gluons with opposite helicity. Reverting the argument, the allowed helicity configurations in SYM constrain the allowed amplitudes in superstring theory.

\section{Soft limit}

\subsection{General arguments}

In [14] the soft limits of massless string amplitudes was studied both explicitly (up to 6point amplitudes) and abstractly by making use of OPE analysis. The conclusion was that disk amplitudes of gluons behave exactly as in Yang-Mills theory at tree level both for the open superstring and for the open bosonic string. Indeed one expects universal behaviour at leading $\left(\delta^{-1}\right)$ and sub-leading $\left(\delta^{0}\right)$ order, in formulae

$$
\begin{aligned}
& \mathcal{A}_{n}(1,2, \ldots, s, \ldots, n)= \\
& \left\{\left[\frac{a_{s} \cdot k_{s+1}}{k_{s} \cdot k_{s+1}}-\frac{a_{s} \cdot k_{s-1}}{k_{s} \cdot k_{s-1}}\right]+\left[\frac{f_{s}: J_{s+1}}{2 k_{s} \cdot k_{s+1}}-\frac{f_{s}: J_{s-1}}{2 k_{s} \cdot k_{s-1}}\right]\right\} \mathcal{A}_{n-1}(1,2, \ldots \hat{s} \ldots, n)+\mathcal{O}(\delta)
\end{aligned}
$$


where $J_{i}$ denotes the angular momentum operator acting on particle $i$ and $f_{s}^{\mu \nu}=k_{s}^{\mu} a_{s}^{\nu}-$ $k_{s}^{\nu} a_{s}^{\mu}$, as by now usual.

We would like to extend the analysis of [14] based on the OPE to open string amplitudes with massive states.

The leading and subleading soft behaviours are captured by the OPE of the soft gluon integrated vertex with the adjacent (integrated) vertices. Using

$$
\int^{z_{s+1}} d z_{s}\left(z_{s+1}-z_{s}\right)^{2 \alpha^{\prime} k_{s} k_{s+1}-1} F\left(z_{s}, z_{i}\right) \approx \frac{F\left(z_{s+1}, z_{i}\right)}{2 \alpha^{\prime} k_{s} k_{s+1}}+\ldots
$$

and similarly for $z_{s-1}$ one gets

$$
V_{A}\left(a_{s}, k_{s}\right) V_{A}\left(a_{s \pm 1}, k_{s \pm 1}\right) \approx \pm \frac{a_{s} k_{s \pm 1}}{2 k_{s} k_{s \pm 1}} V_{A}\left(a_{s \pm 1}, k_{s}+k_{s \pm 1}\right)+\ldots
$$

where ... includes massive string states which do not contribute to the leading singularity since

$$
V_{A}\left(a_{s}, k_{s}\right) V_{A}\left(a_{s \pm 1}, k_{s \pm 1}\right) \approx \ldots+\sum_{M \neq 0} \frac{1}{2 k_{s} k_{s \pm 1}+M_{H}^{2}} V_{M}\left(H\left[a_{s}, a_{s \pm 1}, k_{s}, k_{s \pm 1}\right], k_{s}+k_{s \pm 1}\right)+\ldots
$$

where $V_{M}$ denotes the vertex operator of a massive state, with momentum $p=k_{s}+k_{s \pm 1}$ and polarisation $H$ that can be expressed in terms of $a_{s}, a_{s \pm 1}, k_{s}, k_{s \pm 1}$.

Expanding the denominator as

$$
\frac{1}{2 k_{s} k_{s \pm 1}+M_{H}^{2}} \approx \frac{1}{M_{H}^{2}}\left(1-\frac{2 k_{s} k_{s \pm 1}}{M_{H}^{2}}+\ldots\right)
$$

one immediately sees that at most the sub-leading (regular $\delta^{0}$ behaviour) might be affected. However the tri-linear coupling $A-A-H$ contains at least one soft momentum $k_{s}$ and this produces a further suppression by $\delta^{+1}$. This holds true also for the tachyon since the $T-A-A$ coupling involves two momenta $\mathcal{A}_{T-A-A}=T\left(k_{1} k_{2} a_{1} a_{2}-k_{1} a_{2} k_{2} a_{1}\right)$, similarly for $H_{\mu \nu}$ since $\mathcal{A}_{H-A-A}=H_{\mu}{ }^{\nu} f_{\nu}^{1 \rho} f_{\rho}^{2 \mu}+\ldots$, while for $C_{\mu \nu \rho}$ at the first massive level of the superstring one has $\mathcal{A}_{C-A-A}=C_{\mu \nu \rho} a_{1}^{\mu} a_{2}^{\nu}\left(k_{1}-k_{2}\right)^{\rho}$.

Let us now consider the case where the soft gluon is attached to a massive (or tachyonic) leg

$$
V_{A}\left(a_{s}, k_{s}\right) V_{M}\left(H_{s \pm 1}, p_{s \pm 1}\right) \approx \frac{1}{2 k_{s} p_{s \pm 1}} V_{M^{\prime}}\left(H^{\prime}\left[a_{s}, H_{s \pm 1}, k_{s}, p_{s \pm 1}\right], k_{s}+p_{s \pm 1}\right)+\ldots
$$

where $M^{\prime}$ denotes any state at the same mass level as the state $M$. For the bosonic string at the tachyonic and first massive level only one kind of particles appears so much so that (for totally symmetric tensors of the first Regge trajectory at level $N=\ell-1$ )

$$
\begin{aligned}
& \mathcal{A}_{A H_{\ell} H_{\ell}}= \\
& \left.a_{1} p_{23} H_{2}^{\mu_{1} \ldots \mu_{\ell}} H_{3, \mu_{1} \ldots \mu_{\ell}}+a_{1, \mu} H_{2}^{\mu \mu_{2} \ldots \mu_{\ell}} p_{12}^{\nu} H_{3, \nu \mu_{2} \ldots \mu_{\ell}}+p_{31, \mu} H_{2}^{\mu \mu_{2} \ldots \mu_{\ell}} a_{1}^{\nu} H_{3, \nu \mu_{2} \ldots \mu_{\ell}}+\mathcal{O}\left(\alpha^{\prime} p^{2}\right)\right]
\end{aligned}
$$


The first term is the string analogue of minimal coupling that is leading in the soft limit $k_{1} \rightarrow 0$. Gauge invariance fixes the sub-leading term to be as expected. Indeed, for color-ordered amplitudes with $n+1$ gluons and no massive states one finds [14, 22]

$$
\begin{aligned}
& \mathcal{A}_{n+1}(1, \ldots s \ldots, n+1)= \\
& \left.\quad \frac{ \pm 1}{2 k_{s} \cdot k_{s \pm 1}} \mathcal{A}_{n}(1, \ldots \hat{s}, \ldots, n+1)\right|_{k_{s+1}^{\prime}=k_{s+1}+k_{s}} ^{a_{s+1}^{\prime}=a_{s+1} a_{s} \cdot k_{s \pm 1}-a_{s} k_{s} \cdot a_{s \pm 1}+k_{s} a_{s} \cdot a_{s \pm 1}}+\ldots
\end{aligned}
$$

expanding in $k_{s}$ yields

$$
\begin{aligned}
\pm & \left\{\frac{a_{s} \cdot k_{s \pm 1}}{2 k_{s} \cdot k_{s \pm 1}}-\frac{k_{s} \cdot a_{s \pm 1}}{2 k_{s} \cdot k_{s \pm 1}} a_{s} \cdot \frac{\partial}{\partial a_{s \pm 1}}+\frac{a_{s} \cdot k_{s \pm 1}}{2 k_{s} \cdot k_{s \pm 1}} k_{s} \cdot \frac{\partial}{\partial k_{s \pm 1}}+\frac{a_{s} \cdot a_{s \pm 1}}{2 k_{s} \cdot k_{s \pm 1}} k_{s} \cdot \frac{\partial}{\partial a_{s \pm 1}}\right\} \\
& \mathcal{A}_{n}(1, \ldots \hat{s} \ldots, n+1)+\ldots
\end{aligned}
$$

gauge invariance dictates the presence of the additional sub-leading term

$$
\mp \frac{k_{s} \cdot k_{s \pm 1}}{2 k_{s} \cdot k_{s \pm 1}} a_{s} \cdot \frac{\partial}{\partial k_{s \pm 1}} \mathcal{A}_{n}(1, \ldots \hat{s} \ldots, n+1)
$$

that completes at sub-leading order the action of $f_{s}: J_{s \pm 1}$ on $\mathcal{A}_{n}(1, \ldots \hat{s} \ldots, n+1)$.

Including $m$ massive states, if the soft gluon is adjacent to two hard gluons the above analysis continues to apply. When at least one of the adjacent legs is massive, let's say the one in position $s+1$, with spin $\ell$ one has

$\mathcal{A}_{n+1, m}(1, \ldots s \ldots, n+m+1)=\left.\frac{ \pm 1}{2 k_{s} \cdot p_{s+1}} \mathcal{A}_{n, m}(1, \ldots \hat{s} \ldots, n+m+1)\right|_{p_{s+1}^{\prime}=p_{s+1}+k_{s}} ^{H_{s+1}^{\prime}=H_{s+1} a_{s} \cdot p_{s+1}+\ldots}+\ldots$

where ... denotes the additional terms in the tri-linear $V-H-H$ coupling. Barring a couple of subtleties, we will deal with later on, expanding in $k_{s}$ one gets (schematically)

$$
\begin{aligned}
\pm & \left\{\frac{a_{s} \cdot p_{s+1}}{2 k_{s} \cdot p_{s+1}}-\ell \frac{k_{s} \cdot H_{s+1}}{2 k_{s} \cdot p_{s+1}} a_{s} \cdot \frac{\partial}{\partial H_{s+1}}+\frac{a_{s} \cdot p_{s+1}}{2 k_{s} \cdot p_{s+1}} k_{s} \cdot \frac{\partial}{\partial p_{s+1}}+\ell \frac{a_{s} \cdot H_{s+1}}{2 k_{s} \cdot p_{s+1}} k_{s} \cdot \frac{\partial}{\partial H_{s+1}}\right\} \\
& \mathcal{A}_{n, m}(1, \ldots \hat{s} \ldots, n+m+1)+\ldots
\end{aligned}
$$

gauge invariance w.r.t. the soft gluon dictates the presence of the additional subleading term

$$
\mp \frac{k_{s} \cdot p_{s+1}}{2 k_{s} \cdot p_{s+1}} a_{s} \cdot \frac{\partial}{\partial p_{s+1}} \mathcal{A}_{n, m}(1, \ldots \hat{s} \ldots, n+m+1)
$$

that completes the action of $f_{s}: J_{s+1}$ on $\mathcal{A}_{n, m}(1, \ldots \hat{s} \ldots, n+m+1)$ at sub-leading order.

Now let us deal with two subtleties: the higher derivative terms in the tri-linear coupling $A-H-H$ and the possible non-diagonal couplings $A-H-H^{\prime}$ that would spoil universality. The former is easy to dispose of, higher derivative corrections to minimal coupling can only affect the sub-leading term that is fixed by gauge invariance w.r.t. the soft gluon starting from the low-derivative terms coded in the OPE. The latter requires more attention. For open superstrings, as we have seen, already at the first massive level one finds two kinds of particles in the Neveu-Schwarz sector: $C_{\mu \nu \rho}$ (3-index anti-symmetric tensor, 84 d.o.f.) and $H_{\mu \nu}$ (2-index symmetric traceless tensor, 44 d.o.f.). In addition to the 'diagonal' couplings 
$V-C-C$ and $V-H-H$ (and SUSY related) one should consider the mixed coupling $V-H-C$ $\approx \alpha^{\prime} M p_{31} \cdot H_{2} \cdot C_{3}:\left[a_{1} p_{12}\right]$ that exposes the singular soft factor $1 / k p$ since $M_{C}=M_{H}$ but gets suppressed by an extra power of the soft momentum in the numerator. Lacking the leading $\delta^{-1}$ term that fixes also the sub-leading $\delta^{0}$ term, thanks to gauge invariance, this kind of higher derivative non-diagonal couplings can at most affect the sub-sub-leading $\delta^{+1}$ (and higher) terms which are not expected to be universal. Although the situation gets exponentially more intricate the higher the mass level and spin, we conclude that no correction are to be expected w.r.t. the standard YM case in the soft behaviour for open string amplitudes involving massive states.

For illustrative purposes, we will explicitly check the above statements in the soft limit of some 4-point amplitudes with massive string states at the first level. Differently to the case of amplitudes with only mass-less external states that factorise on 3-point amplitudes, that would vanish for real momenta due to collinearity, when some of the external states are massive, the soft limit can produce physical 3-point amplitudes e.g. widths or decay rates of massive states into lower mass ones.

4.2 Soft limit of $\mathcal{A}\left(A_{1}, T_{2}, T_{3}, T_{4}\right)$

In this case the limit $k_{1} \rightarrow 0$ is straightforward. Consider first the expansion of the factor

$$
\frac{\Gamma\left(1+2 \alpha^{\prime} k_{1} p_{2}\right) \Gamma\left(1+2 \alpha^{\prime} k_{1} p_{4}\right)}{\Gamma\left(1-2 \alpha^{\prime} k_{1} p_{3}\right)}=\frac{\left(1+2 \alpha^{\prime} k_{1} p_{2} \psi(1)\right)\left(1+2 \alpha^{\prime} k_{1} p_{4} \psi(1)\right)}{1-2 \alpha^{\prime} k_{1} p_{3} \psi(1)}=1+\mathcal{O}\left(\delta^{2}\right) .
$$

The expansion of the full amplitude reads

$$
\mathcal{A}\left(A_{1}, T_{2}, T_{3}, T_{4}\right) \propto\left(\frac{a_{1} p_{2}}{k_{1} p_{2}}-\frac{a_{1} p_{4}}{k_{1} p_{4}}\right) \mathcal{A}\left(T_{2}, T_{3}, T_{4}\right)+\mathcal{O}(\delta)
$$

showing the expected singular behavior in both the $s$ and the $t$ channels, whereas the term of order $\mathcal{O}\left(\delta^{0}\right)$ vanishes because

$$
\frac{1}{2} f_{1}^{\mu \nu} J_{i \mu \nu} \mathcal{A}\left(T_{2}, T_{3}, T_{4}\right)=0, \quad i=2,4 .
$$

being the 3-tachyon amplitude a constant independent from the momenta.

\subsection{Soft limit of $\mathcal{A}\left(A_{1}, A_{2}, T_{3}, T_{4}\right)$}

Consider the amplitude in eq. (B.4) once we have expressed the Euler beta function in terms of Euler gamma functions

$$
\begin{aligned}
& \frac{\Gamma\left(1+2 \alpha^{\prime} k_{1} p_{4}\right) \Gamma\left(1+2 \alpha^{\prime} k_{1} k_{2}\right)}{\Gamma\left(1-2 \alpha^{\prime} k_{1} p_{3}\right)} \frac{1}{1-2 \alpha^{\prime} k_{1} k_{2}}\left(\frac { k _ { 1 } p _ { 3 } } { k _ { 1 } k _ { 2 } } \left(-a_{1} a_{2}+2 \alpha^{\prime}\left(a_{1} p_{3} a_{2} p_{3}+a_{1} p_{4} a_{2} p_{4}\right)\right.\right. \\
& \left.-a_{1} p_{3} a_{2} p_{4} \frac{1+2 \alpha^{\prime} k_{1} p_{4}}{k_{1} k_{2}}-a_{1} p_{4} a_{2} p_{3} \frac{1+2 \alpha^{\prime} k_{1} p_{3}}{k_{1} p_{4} k_{1} k_{2}} k_{1} p_{3}\right) .
\end{aligned}
$$

As already verified, the combination of Euler gamma functions in the above expression contributes in the limit $\delta \rightarrow 0$ as $1+\mathcal{O}\left(\delta^{2}\right)$, thus can be neglected. We have two terms of order $\delta^{-1}$ :

$$
-\frac{a_{1} p_{3} a_{2} p_{4}}{k_{1} k_{2}}-\frac{a_{1} p_{4} a_{2} p_{3} k_{1} p_{3}}{k_{1} k_{1} k_{1} p_{4}}
$$


Using the identity

$$
\frac{k_{1} p_{3}}{k_{1} k_{2} k_{1} p_{4}}=-\frac{1}{k_{1} k_{2}}-\frac{1}{k_{1} p_{4}},
$$

eq. (4.18) can be written as

$$
\begin{aligned}
& \frac{a_{1} p_{4} a_{2} p_{3}-a_{1} p_{3} a_{2} p_{4}}{k_{1} k_{2}}+\frac{a_{1} p_{4} a_{2} p_{3}}{k_{1} p_{4}}=\left(\frac{1}{2} a_{1} p_{+} a_{2} p_{-}-\frac{1}{2} a_{1} p_{-} a_{2} p_{+}\right) \frac{1}{k_{1} k_{2}} \\
& +\left(a_{1} \frac{p_{+}-p_{-}}{2} a_{2} \frac{p_{-}}{2}+a_{1} \frac{p_{+}-p_{-}}{2} a_{2} \frac{p_{+}}{2}\right) \frac{1}{k_{1} p_{4}} \\
& \quad=\left(-\frac{a_{1} k_{2}}{k_{1} k_{2}}+\frac{a_{1} p_{4}}{k_{1} p_{4}}\right) a_{2} \frac{p_{34}}{2}+\frac{1}{2} \frac{a_{1} p_{-} a_{2} k_{1}}{k_{1} k_{2}}-\frac{1}{2} \frac{a_{1} p_{4} a_{2} k_{1}}{k_{1} p_{4}},
\end{aligned}
$$

where

$$
p_{+}=p_{3}+p_{4} \quad p_{-}=p_{3}-p_{4} .
$$

The leading soft contribution is, as expected,

$$
\left(-\frac{a_{1} k_{2}}{k_{1} k_{2}}+\frac{a_{1} p_{4}}{k_{1} p_{4}}\right) \mathcal{A}\left(A_{2}, T_{3}, T_{4}\right) .
$$

The order $\mathcal{O}\left(\delta^{0}\right)$ contribution to the amplitude reads

$$
\begin{aligned}
& -a_{1} a_{2} \frac{k_{1} p_{3}}{k_{1} k_{2}}+\frac{1}{2} \frac{a_{1} p_{-} a_{2} k_{1}}{k_{1} k_{2}}-\frac{1}{2} \frac{a_{1} p_{4} a_{2} k_{1}}{k_{1} p_{4}}+2 \alpha^{\prime}\left(\frac{k_{1} p_{3}}{k_{1} k_{2}}\left(a_{1} p_{3} a_{2} p_{3}+a_{1} p_{4} a_{2} p_{4}+a_{1} p_{4} a_{2} p_{3}\right)\right. \\
& \left.+a_{1} p_{4} a_{2} p_{3}\left(\frac{k_{1} p_{3}}{k_{1} p_{4}}+\frac{k_{1} k_{2}}{k_{1} p_{4}}+1\right)-a_{1} p_{3} a_{2} p_{4}\left(\frac{k_{1} p_{4}}{k_{1} k_{2}}+1\right)\right) \\
& =-a_{1} a_{2} \frac{k_{1} p_{3}}{k_{1} k_{2}}+\frac{1}{2} \frac{a_{1} p_{-} a_{2} k_{1}}{k_{1} k_{2}}-\frac{1}{2} \frac{a_{1} p_{4} a_{2} k_{1}}{k_{1} p_{4}}+2 \alpha^{\prime} a_{1} p_{+} a_{2} p_{+} \\
& =-\frac{1}{2} a_{1} a_{2} \frac{k_{1} p_{3}}{k_{1} k_{2}}+\frac{1}{2} a_{1} a_{2} \frac{k_{1} p_{4}}{k_{1} k_{2}}+\frac{1}{2} a_{1} a_{2} \frac{k_{1} p_{4}}{k_{1} p_{4}}+\frac{1}{2} \frac{a_{1} p_{-} a_{2} k_{1}}{k_{1} k_{2}}-\frac{1}{2} \frac{a_{1} p_{4} a_{2} k_{1}}{k_{1} p_{4}}+\mathcal{O}(\delta) .
\end{aligned}
$$

In the above expression we recognize the expected behavior

$$
\begin{aligned}
\frac{1}{2 k_{1} k_{2}} f_{1 \mu \nu} J_{2}^{\mu \nu} \mathcal{A}\left(V_{2}, T_{3}, T_{4}\right) & =\left(a_{1} a_{2} k_{1} \frac{p_{34}}{2}-a_{1} \frac{p_{34}}{2} a_{2} k_{1}\right) \frac{1}{k_{1} k_{2}}, \\
\frac{1}{2 k_{1} p_{4}} f_{1 \mu \nu} J_{4}^{\mu \nu} \mathcal{A}\left(A_{2}, T_{3}, T_{4}\right) & =\left(\frac{1}{2} a_{1} a_{2} k_{1} p_{4}-a_{1} p_{4} a_{2} k_{1}\right) \frac{1}{k_{1} p_{4}} .
\end{aligned}
$$

\subsection{Soft limit of $\mathcal{A}\left(T_{1}, T_{2}, A_{3}, H_{4}\right)$}

Consider the amplitude in eq. (B.6). Let us first discuss the soft limit of the kinematical factor

$$
\begin{aligned}
& \frac{\Gamma\left(-1+2 \alpha^{\prime} k_{3} p_{4}\right) \Gamma\left(1+2 \alpha^{\prime} p_{3} k_{3}\right)}{\Gamma\left(-2 \alpha^{\prime} p_{1} k_{3}\right)}=\frac{\Gamma\left(1+2 \alpha^{\prime} k_{3} p_{4}\right) \Gamma\left(1+2 \alpha^{\prime} p_{3} k_{3}\right)}{\Gamma\left(1-2 \alpha^{\prime} p_{1} k_{3}\right)} \frac{k_{3} p_{1}}{k_{3} p_{4}\left(1-2 \alpha^{\prime} k_{3} p_{4}\right)} \\
& \quad=\left(1+\mathcal{O}\left(\delta^{2}\right)\right) \frac{k_{3} p_{1}}{k_{3} p_{4}} \frac{1}{1-2 \alpha^{\prime} k_{3} p_{4}} .
\end{aligned}
$$


It is then convenient to multiply the tensorial part of the amplitude for the above expansion in order to identify more easily the contributions up to $\mathcal{O}\left(\delta^{0}\right)$ :

$$
\begin{aligned}
\frac{a_{3} p_{4}}{k_{3} p_{4}} p_{2} H p_{2} & =\frac{a_{3} p_{4}}{k_{3} p_{4}} \frac{p_{12}}{2} H \frac{p_{12}}{2}+\mathcal{O}\left(\delta^{0}\right), \quad \mathcal{O}\left(\delta^{0}\right)=\frac{a_{3} p_{4}}{k_{3} p_{4}} k_{3} H \frac{p_{12}}{2} ; \\
-\frac{a_{3} p_{2}}{k_{3} p_{2}} p_{2} H p_{2} & =-\frac{a_{3} p_{2}}{k_{3} p_{2}} \frac{p_{12}}{2} H \frac{p_{12}}{2}+\mathcal{O}\left(\delta^{0}\right), \quad \mathcal{O}\left(\delta^{0}\right)=-\frac{a_{3} p_{2}}{k_{3} p_{2}} k_{3} H \frac{p_{12}}{2} ; \\
2 a_{3} p_{2} p_{2} H k_{3} \frac{p_{1} k_{3}}{p_{2} k_{3} k_{3} p_{4}} & =-2 \frac{a_{3} p_{2}}{p_{2} k_{3}} p_{2} H k_{3}-2 \frac{a_{3} p_{2}}{k_{3} p_{4}} p_{2} H k_{3} ; \\
-2 a_{3} H p_{2} \frac{p_{1} k_{3}}{k_{3} p_{4}} \frac{p_{2} k_{3}}{p_{2} k_{3}} & =2 a_{3} H p_{2}+2 \frac{k_{3} p_{2}}{k_{3} p_{4}} a_{3} H p_{2} .
\end{aligned}
$$

The leading order $\mathcal{O}\left(\delta^{-1}\right)$ behaves as expected

$$
\mathcal{A}^{\delta^{-1}}\left(T_{1}, T_{2}, A_{3}, H_{4}\right)=\left(\frac{a_{3} p_{4}}{k_{3} p_{4}}-\frac{a_{3} p_{2}}{k_{3} p_{2}}\right) \frac{p_{12}}{2} H \frac{p_{12}}{2},
$$

being $\mathcal{A}\left(T_{1}, T_{2}, H_{4}\right)=\frac{p_{12}}{2} H \frac{p_{12}}{2}$. Look at the subleading contribution:

$$
\begin{aligned}
& \frac{1}{k_{3} p_{2}}\left(-a_{3} p_{2} k_{3} H \frac{p_{12}}{2}-2 a_{3} p_{2} p_{3} H k_{3}+a_{3} H p_{2} k_{3} p_{2}\right) \\
& \frac{1}{k_{3} p_{4}}\left(a_{3} p_{4} k_{3} H \frac{p_{12}}{2}-2 a_{3} p_{2} p_{3} H k_{3}+a_{3} H p_{2}\left(2 k_{3} p_{2}+k_{3} p_{4}\right)\right) \\
& =\frac{1}{k_{3} p_{2}}\left(a_{3} p_{2} k_{3} H \frac{p_{12}}{2}-k_{3} p_{2} a_{3} H \frac{p_{12}}{2}\right)-\frac{1}{k_{3} p_{4}}\left(2 a_{3} \frac{p_{12}}{2} k_{3} H \frac{p_{12}}{2}-2 k_{3} \frac{p_{12}}{2} a_{3} H \frac{p_{12}}{2}\right) .
\end{aligned}
$$

It is easy to verify that the above expressions coincide with:

$$
\frac{1}{2 k_{3} p_{i}} f_{3 \mu \nu} J_{i}^{\mu \nu} \mathcal{A}\left(T_{1}, T_{2}, H_{4}\right), \quad i=2,4 .
$$

We recall that the angular momentum for a spin-2 particle takes the form

$$
J_{\mu \nu}=p_{\mu} \frac{\partial}{\partial p^{\nu}}-p_{\nu} \frac{\partial}{\partial p^{\mu}}+2 H_{\mu \lambda} \frac{\partial}{\partial H_{\lambda}^{\nu}}-2 H_{\nu \lambda} \frac{\partial}{\partial H_{\lambda}^{\mu}} .
$$

\subsection{Soft limit of $\mathcal{A}\left(A_{1}, A_{2}, A_{3}, C_{4}\right)$}

The amplitude is given in Eq. (3.36). Let us study the soft behaviour when $k_{3} \rightarrow 0$. Recall that $s+t+u=1 / \alpha^{\prime}$

The Veneziano factor $\mathcal{B}(1,1)$ yields

$$
\mathcal{B}(1,1)=\frac{\Gamma\left(1-\alpha^{\prime} s\right) \Gamma\left(1-\alpha^{\prime} t\right)}{\Gamma\left(1+\alpha^{\prime} u\right)}=\frac{\Gamma\left(2 \alpha^{\prime} k_{3} p_{4}\right) \Gamma\left(1+2 \alpha^{\prime} k_{3} k_{2}\right)}{\Gamma\left(1-2 \alpha^{\prime} k_{3} k_{1}\right)} \approx \frac{1}{2 \alpha^{\prime} k_{3} p_{4}} \times\left[1+\mathcal{O}\left(\delta^{2}\right)\right]
$$

To leading order the polarisation dependent factor yields

$$
\mathcal{P}=-a_{1} \cdot C_{4}: f_{2}\left(a_{3} \cdot k_{1}-\frac{k_{3} \cdot k_{1}}{k_{3} \cdot k_{2}} a_{3} \cdot k_{2}\right)
$$

combining the two one gets, as expected

$$
\mathcal{A}_{4}^{\delta^{-1}}\left(A_{1}, A_{2}, A_{3}, C_{4}\right)=\left(\frac{a_{3} \cdot p_{4}}{k_{3} p_{4}}-\frac{a_{3} \cdot k_{2}}{k_{3} \cdot k_{2}}\right) a_{1} \cdot C_{4}: f_{2}=\left(\frac{a_{3} \cdot p_{4}}{k_{3} p_{4}}-\frac{a_{3} \cdot k_{2}}{k_{3} \cdot k_{2}}\right) \mathcal{A}_{3}\left(A_{1}, A_{2}, C_{4}\right)
$$


To sub-leading order $\delta^{0}$, one gets

$$
\begin{aligned}
\mathcal{A}_{4}^{\delta^{0}}\left(A_{1}, A_{2}, A_{3}, C_{4}\right) & =\left(\frac{2 a_{1} \cdot C_{4} \cdot f_{3} \cdot f_{2}-a_{1} \cdot f_{3} \cdot C_{4} \cdot f_{2}}{k_{3} p_{4}}-\frac{2 a_{1} \cdot C_{4} \cdot f_{3} \cdot f_{2}}{k_{3} \cdot k_{2}}\right) \\
& =\left(\frac{f_{3}: J_{4}}{k_{3} p_{4}}-\frac{f_{3}: J_{2}}{k_{3} \cdot k_{2}}\right) \mathcal{A}_{3}\left(A_{1}, A_{2}, C_{4}\right)
\end{aligned}
$$

as expected, where

$J_{2}{ }^{\mu}{ }_{\nu}=\frac{k_{2}^{\mu} \partial}{\partial k_{2}^{\nu}}-\frac{k_{2}^{\mu} \partial}{\partial k_{2}^{\nu}}+a_{2}^{\mu} \frac{\partial}{\partial a_{2}^{\nu}}-a_{2}^{\mu} \frac{\partial}{\partial a_{2}^{\nu}}, \quad J_{4}{ }^{\mu}{ }_{\nu}=p_{4}^{\mu} \frac{\partial}{\partial p_{4}^{\nu}}-p_{4}^{\mu} \frac{\partial}{\partial p_{4}^{\nu}}+3 C_{4}^{\mu \lambda \rho} \frac{\partial}{\partial C_{4}^{\nu \lambda \rho}}-3 C_{4}^{\nu \lambda \rho} \frac{\partial}{\partial C_{4}^{\mu \lambda \rho}}$

Actually $\partial / \partial p_{4}$ acts trivially in this case].

With little more effort one would get the same result for $k_{1} \rightarrow 0$, while for $k_{2} \rightarrow 0$ the only contributions come from 'standard' soft behaviour of gluons hitting adjacent gluons.

This gives support to our general conclusion that superstring amplitudes with $n$ massless and $m$ massive external legs on the boundary of the disk behave universally in the soft limit.

\subsection{Soft limit of $\mathcal{A}\left(A_{1}, A_{2}, A_{3}, H_{4}\right)$}

The amplitude is given in Eq. (3.43). Let us study the soft behaviour when $k_{3} \rightarrow 0$. Recall that $s+t+u=1 / \alpha^{\prime}$ as for $\mathcal{A}\left(A_{1}, A_{2}, A_{3}, C_{4}\right)$. Following the same steps one finds to leading order

$$
\begin{gathered}
\mathcal{A}_{4}^{\delta^{-1}}\left(A_{1}, A_{2}, A_{3}, H_{4}\right)=\frac{k_{3} k_{1}}{k_{3} p_{4}}\left(\frac{a_{3} k_{2}}{k_{3} k_{2}}-\frac{a_{3} k_{1}}{k_{3} k_{1}}\right)\left[\frac{1}{2} m_{H}^{2} a_{1} H a_{2}+a_{2} k_{1} a_{1} H k_{2}-a_{1} f_{2} H k_{2}\right]= \\
-\left(\frac{a_{3} k_{2}}{k_{3} k_{2}}-\frac{a_{3} p_{4}}{k_{3} p_{4}}\right) f_{1} H f_{2}+\mathcal{O}(1)=\left(\frac{a_{3} k_{2}}{k_{3} k_{2}}-\frac{a_{3} p_{4}}{k_{3} p_{4}}\right) \mathcal{A}_{3}\left(A_{1}, A_{2}, H_{4}\right)
\end{gathered}
$$

where use of $m_{H}^{2}=-p_{4}^{2}=-2 k_{1} k_{2}+\mathcal{O}(\delta)$ has been made.

At subleading order one finds several terms i.e.

$$
\begin{aligned}
& \frac{1}{k_{3} k_{2}}\left[a_{2} a_{3}\left(k_{3} p_{4} a_{1} H k_{2}-a_{1} H k_{3} \frac{m_{H}^{2}}{2}\right)+a_{1} H a_{3} a_{2} k_{3} \frac{m_{H}^{2}}{2}+a_{1} H k_{2} a_{2} k_{3} a_{3} k_{1}\right. \\
& -a_{1} H k_{3} a_{2} k_{1} a_{3} k_{2}-a_{1} f_{2} f_{3} H k_{2}+a_{1} f_{3} f_{2} k_{2}-k_{3} p_{4} a_{1} H a_{2} a_{3} k_{2}+a_{3} p_{4} a_{2} H a_{2} k_{2} k_{3} \\
& \left.+a_{2} a_{3} a_{1} H k_{2} k_{2} k_{3}+a_{2} k_{1} a_{1} H a_{3} k_{2} k_{3}\right] \\
& +\frac{1}{k_{3} p_{4}}\left[a_{2} a_{3} a_{1} H k_{3} \frac{m_{H}^{2}}{2}+a_{1} H a_{3} a_{2} k_{3} \frac{m_{H}^{2}}{2}+a_{1} H a_{3} a_{2} k_{1} k_{3} k_{2}-a_{1} f_{2} H a_{3} k_{3} k_{1}-a_{1} f_{3} H a_{2}\right. \\
& \left.-a_{1} H k_{3} a_{2} k_{1} a_{3} k_{2}-a_{1} f_{2} H k_{3} a_{3} k_{2}-a_{1} f_{3} H k_{2} a_{2} k_{1}-a_{1} f_{2} f_{3} H k_{2}-a_{3} p_{4} a_{1} f_{2} H k_{3}\right]
\end{aligned}
$$

Summing the terms within squared parenthesis everything can be written in terms of $f_{1}, f_{2}, f_{3}$ (as dictated by gauge invariance at this order) finding

$$
\begin{aligned}
\mathcal{A}_{4}^{\delta^{0}}\left(A_{1}, A_{2}, A_{3}, H_{4}\right) & =\left(\frac{f_{1} H f_{3} f_{2}-f_{1} H f_{2} f_{3}}{k_{3} k_{2}}-\frac{f_{1} f_{3} H f_{2}-f_{1} H f_{3} f_{2}}{k_{3} p_{4}}\right) \\
& =\left(\frac{f_{3} J_{2}}{k_{3} k_{2}}-\frac{f_{3} J_{4}}{k_{3} p_{4}}\right) \mathcal{A}_{3}\left(A_{1}, A_{2}, H_{4}\right)
\end{aligned}
$$


with ${J_{2}}^{\mu}{ }_{\nu}$ given above and

$$
{J_{4}^{H}}^{\mu^{\mu}}=p_{4}^{\mu} \frac{\partial}{\partial p_{4}^{\nu}}-p_{4}^{\mu} \frac{\partial}{\partial p_{4}^{\nu}}+2 H_{4}^{\mu \lambda} \frac{\partial}{\partial H_{4}^{\nu \lambda}}-2 H_{4}^{\nu \lambda} \frac{\partial}{\partial H_{4}^{\mu \lambda}}
$$

As above $\partial / \partial p_{4}$ acts trivially in this case. This gives further support to our general arguments on the soft limit.

\section{Holomorphic soft limit}

In this section we verify that open string amplitudes with massive external states enjoy the same universal behaviour as YM amplitudes in the holomorphic soft limit [12]. In this limit the holomorphic spinor $u_{s}$ of a positive helicity gluon (inserted between leg 1 and leg $n$ ) is scaled to zero $u=\delta \hat{u}_{s}$. In SYM the leading behaviour as $\delta^{-2}$ is governed by the operator

$$
S_{Y M}^{0}=\frac{\langle n 1\rangle}{\langle n s\rangle\langle s 1\rangle}
$$

the sub-leading behaviour as $\delta^{-1}$ is governd by the operator

$$
S_{Y M}^{1}=\frac{\langle n 1\rangle}{\langle n s\rangle\langle s 1\rangle}\left\{\frac{\langle s n\rangle}{\langle 1 n\rangle} \bar{u}_{s}^{\dot{\alpha}} \frac{\partial}{\partial \bar{u}_{1}^{\dot{\alpha}}}+\frac{\langle s 1\rangle}{\langle n 1\rangle} \bar{u}_{s}^{\dot{\alpha}} \frac{\partial}{\partial \bar{u}_{n}^{\dot{\alpha}}}\right\}
$$

For MHV amplitude the sub-leading term vanishes and the procedure exponentiates [12]. In general it is convenient to use momentum conservation to express two $\bar{u}$ 's in terms of the remaining ones and the $u$ 's. In our case, an obvious choice is to express $\bar{u}_{4}$ and $\bar{u}_{5}$ that appear in the definition of the massive momentum $p=k_{4}+k_{5}=u_{4} \bar{u}_{4}+u_{5} \bar{u}_{5}$. When taking derivatives one has to take into account the mass constraint $m^{2}=-\left(k_{4}+k_{5}\right)^{2}$ as we will see momentarily.

\section{$5.1 \mathcal{A}\left(A^{+}, A^{+}, A^{+}, C\right)$}

Consider the amplitude

$$
\mathcal{A}\left(1^{+}, 2^{+}, 3^{+}, C_{0}\right)=B\left(1-\alpha^{\prime} s, 1-\alpha^{\prime} t\right) \frac{[13] m_{C}}{\langle 12\rangle\langle 23\rangle}
$$

and take the limit for $u_{2} \rightarrow \delta u_{2}$, with $\delta \rightarrow 0$. It is straightforward to show that

$$
B\left(1-\alpha^{\prime} s, 1-\alpha^{\prime} t\right)=\frac{1}{\alpha^{\prime}\langle 13\rangle[13]}\left(1+\mathcal{O}\left(\delta^{2}\right)\right) .
$$

The momentum of the massive particle is the sum of two massless momenta $p=k_{4}+k_{5}$ with the constraint $\langle 45\rangle[54]=m_{C}^{2}$. This constraint implies

$$
\begin{aligned}
& m_{C}^{2}=\langle 45\rangle[54]=\langle 13\rangle[13]+\delta(\langle 12\rangle[12]+\langle 23\rangle[23]), \\
& m_{C}=m_{C}(\delta=0)\left(1+\frac{1}{2} \delta\left(\frac{\langle 12\rangle[12]}{\langle 13\rangle[13]}+\frac{\langle 23\rangle[23]}{\langle 13\rangle[13]}\right)\right)+\mathcal{O}\left(\delta^{2}\right) .
\end{aligned}
$$


Expanding the amplitude, one finds

$$
\begin{aligned}
& \mathcal{A}\left(1^{+}, 2^{+}, 3^{+}, C_{0}\right)=\frac{1}{\delta^{2}} \frac{m_{C}^{2}}{\langle 31\rangle[13]} \frac{[13] m_{C}}{\langle 12\rangle\langle 23\rangle}\left(1+\mathcal{O}\left(\delta^{2}\right)\right) \\
& =-(1+\delta(\langle 12\rangle[12]+\langle 23\rangle[23])) \frac{[13] m_{C}}{\langle 12\rangle\langle 23\rangle}\left(1+\frac{\delta}{2\langle 13\rangle[13]}(\langle 12\rangle[12]+\langle 23\rangle[23])\right)+\mathcal{O}\left(\delta^{2}\right) \\
& =-\frac{1}{\delta^{2}} \frac{[13] m_{C}}{\langle 12\rangle\langle 23\rangle}\left(1+\frac{3}{2} \delta\left(\frac{\langle 12\rangle[12]}{\langle 13\rangle[13]}+\frac{\langle 23\rangle[23]}{\langle 13\rangle[13]}\right)\right)
\end{aligned}
$$

The leading contribution to the holomorphic soft limit is easily identified to be

$$
\mathcal{A}^{(-2)}\left(1^{+}, 2^{+}, 3^{+}, C_{0}\right)=\frac{1}{\delta^{2}} \frac{\langle 31\rangle}{\langle 32\rangle\langle 21\rangle} \mathcal{A}\left(1^{+}, 3^{+}, C_{0}\right)=\frac{1}{\delta^{2}} \frac{\langle 31\rangle}{\langle 32\rangle\langle 21\rangle} \frac{[13]^{2}}{m_{C}}=-\frac{[13] m_{C}}{\delta^{2}\langle 12\rangle\langle 23\rangle} .
$$

that meets our expectations.

The sub-leading contribution is expected to be

$$
\mathcal{A}^{(-1)}\left(1^{+}, 2^{+}, 3^{+}, C_{0}\right)=\frac{1}{\delta} \frac{\langle 31\rangle}{\langle 32\rangle\langle 21\rangle}\left(\frac{\langle 23\rangle}{\langle 13\rangle} \bar{u}_{2} \frac{\partial}{\partial \bar{u}_{1}}+\frac{\langle 21\rangle}{\langle 31\rangle} \bar{u}_{2} \frac{\partial}{\partial \bar{u}_{3}}\right) \mathcal{A}\left(1^{+}, 3^{+}, C_{0}\right) .
$$

In the presence of the mass constraint, the derivatives w.r.t. $\bar{u}_{1,3}$ are replaced by

$$
\frac{\partial}{\partial \bar{u}_{1,3}} \rightarrow \frac{d}{d \bar{u}_{1,3}}=\frac{\partial}{\partial \bar{u}_{1,3}}+\frac{\partial m_{C}}{\partial \bar{u}_{1,3}} \frac{\partial}{\partial m_{C}}
$$

with

$$
\left.\left.\frac{\partial m_{C}}{\partial \bar{u}_{1}}=\frac{\langle 13\rangle}{2 m_{C}} \mid 3\right], \quad \frac{\partial m_{C}}{\partial \bar{u}_{3}}=-\frac{\langle 13\rangle}{2 m_{C}} \mid 1\right] .
$$

Writing the three-point function $\mathcal{A}\left(1^{+}, 3^{+}, C_{0}\right)$ in a slightly different way

$$
\frac{[13]^{2}}{m_{C}}=\frac{m_{C}^{3}}{\langle 13\rangle^{2}}+\mathcal{O}(\delta)
$$

we need to evaluate only the derivative of the tri-linear coupling respect to $m_{C}$. Finally we find

$$
\begin{aligned}
\mathcal{A}\left(1^{+}, 2^{+}, 3^{+}, C_{0}\right)^{(-1)} & =\frac{1}{\delta}\left(\frac{\langle 13\rangle[23]}{2\langle 21\rangle m_{C}}-\frac{\langle 13\rangle[21]}{2\langle 32\rangle m_{C}}\right) \frac{\partial}{\partial m_{C}} \mathcal{A}\left(1^{+}, 3^{+}, C_{0}\right) \\
& =-\frac{1}{\delta} \frac{3}{2} \frac{[13] m_{C}}{\langle 12\rangle\langle 23\rangle}\left(\frac{\langle 23\rangle[23]}{\langle 13\rangle[13]}+\frac{\langle 12\rangle[12]}{\langle 13\rangle[13]}\right) .
\end{aligned}
$$

that exposes the expected behaviour, too.

In order to complete our analysis, we consider the case in which the soft momentum is $k_{3}$. Let's first expand the amplitude in eq. (5.1) up to the order $\delta^{-1}$

$$
\mathcal{A}\left(1^{+}, 2^{+}, 3^{+}, C_{0}\right)=\frac{1}{\delta^{2}} \frac{[13] m_{0}^{3}}{2 k_{3} p_{4}\langle 12\rangle\langle 23\rangle}\left(1+\frac{3}{2} \delta \frac{\langle 13\rangle[13]+\langle 23\rangle[23]}{\langle 12\rangle[12]}\right)+\mathcal{O}\left(\delta^{0}\right) .
$$

At leading order, the soft operator is simply

$$
\frac{a_{3}^{+} k_{2}}{2 k_{3} k_{2}}-\frac{a_{3}^{+} p_{4}}{k_{3} p_{4}}
$$


which in the spinor helicity formalism becomes

$$
\frac{\langle q 2\rangle}{\langle 32\rangle\langle 3 q\rangle}-\frac{\langle q 4\rangle[43]+\langle q 5\rangle[53]}{2 k_{3} p_{4}\langle 3 q\rangle}=\frac{\langle 12\rangle[13]}{\langle 23\rangle 2 k_{3} p_{4}},
$$

with the help of Schouten's identity. The expected leading order behavior looks like

$$
\mathcal{A}^{(-2)}\left(1^{+}, 2^{+}, 3^{+}, C_{0}\right)=\frac{1}{\delta^{2}} \frac{\langle 12\rangle[13]}{\langle 23\rangle 2 k_{3} p_{4}} \mathcal{A}\left(1^{+}, 2^{+}, C_{0}\right)=\frac{1}{\delta^{2}} \frac{[13] m_{0}^{3}}{\langle 12\rangle\langle 23\rangle 2 k_{3} p_{4}},
$$

where we exploited the fact that

$$
\mathcal{A}\left(1^{+}, 2^{+}, C_{0}\right)=\frac{[12]^{2}}{m_{0}} .
$$

At sub-leading order we expect the soft operator to be

$$
\frac{f_{3}^{+}: J_{2}}{2 k_{2} k_{3}}-\frac{f_{3}^{+}: J_{4}}{2 k_{3} p_{4}} \rightarrow \frac{1}{\langle 23\rangle} \bar{u}_{3} \frac{d}{d \bar{u}_{2}}-\frac{1}{2 k_{3} p_{4}}\left([34] \bar{u}_{3} \frac{d}{d \bar{u}_{4}}+[35] \bar{u}_{3} \frac{d}{d \bar{u}_{5}}\right) .
$$

Noticing that

$$
\begin{aligned}
& \left(\frac{\partial}{\partial \bar{u}_{2}}+\frac{\partial m_{0}}{\partial \bar{u}_{2}} \frac{\partial}{\partial m_{0}}\right) \frac{[12]^{2}}{m_{0}}=\frac{3}{2} \frac{[12] \bar{u}_{1}}{m_{0}} \\
& \left(\frac{\partial}{\partial \bar{u}_{4}}+\frac{\partial m_{0}}{\partial \bar{u}_{4}} \frac{\partial}{\partial m_{0}}\right) \frac{[12]^{2}}{m_{0}}=\frac{\langle 45\rangle[12]^{2} \bar{u}_{5}}{2 m_{0}^{3}} \\
& \left(\frac{\partial}{\partial \bar{u}_{5}}+\frac{\partial m_{0}}{\partial \bar{u}_{5}} \frac{\partial}{\partial m_{0}}\right) \frac{[12]^{2}}{m_{0}}=-\frac{\langle 45\rangle[12]^{2} \bar{u}_{4}}{2 m_{0}^{3}}
\end{aligned}
$$

we find

$$
\mathcal{A}^{(-1)}\left(1^{+}, 2^{+}, 3^{+}, C_{0}\right)=\frac{3}{2 \delta} \frac{[12][13]}{\langle 23\rangle m_{0}},
$$

which is compatible with eq. (5.15) after noticing that the sub-leading term in the expansion can be written as

$$
\frac{3}{2 \delta} \frac{[13][23][12]}{2 k_{3} p_{4}}+\frac{3}{2 \delta} \frac{[13][23][12]}{m_{0}}\left(-\frac{1}{2 k_{3} p_{4}}+\frac{1}{\langle 23\rangle[23]}\right)=\frac{3}{2 \delta} \frac{[12][13]}{\langle 23\rangle m_{0}} .
$$

$\mathbf{5 . 2} \mathcal{A}\left(A^{-}, A^{+}, A^{-}, C\right)$

Consider now the amplitude

$$
\mathcal{A}\left(1^{-}, 2^{+}, 3^{-}, C_{0}\right)=\frac{\Gamma\left(1+2 \alpha^{\prime} k_{1} k_{2}\right) \Gamma\left(1+2 \alpha^{\prime} k_{2} k_{3}\right)}{\Gamma\left(1-2 \alpha^{\prime} k_{2} p_{4}\right)} \frac{\langle 13\rangle^{3}}{\langle 12\rangle\langle 23\rangle m_{C}} .
$$

Taking the limit in which $u_{2} \rightarrow 0$, we have

$$
\mathcal{A}\left(1^{-}, 2^{+}, 3^{-}, C_{0}\right)=\frac{1}{\delta^{2}} \frac{\langle 13\rangle^{3}}{\langle 12\rangle\langle 23\rangle m_{C}(\delta=0)}\left(1-\frac{\delta}{2}\left(\frac{\langle 12\rangle[12]}{\langle 13\rangle[13]}+\frac{\langle 23\rangle[23]}{\langle 13\rangle[13]}\right)\right) .
$$

For the leading term one finds

$$
\mathcal{A}^{(-2)}\left(1^{-}, 2^{+}, 3^{-}, C_{0}\right)=\frac{1}{\delta^{2}} \frac{\langle 13\rangle}{\langle 12\rangle\langle 23\rangle} \frac{\langle 13\rangle^{2}}{m_{C}},
$$


for the sub-leading term

$$
\mathcal{A}^{(-1)}\left(1^{-}, 2^{+}, 3^{-}, C_{0}\right)=\frac{1}{\delta} \frac{\langle 13\rangle}{\langle 12\rangle\langle 23\rangle} \frac{\langle 13\rangle^{2}}{m_{C}}\left(\frac{\langle 23\rangle\langle 13\rangle}{\langle 13\rangle 2 m_{C}}[23]-\frac{\langle 21\rangle\langle 13\rangle}{\langle 31\rangle 2 m_{C}}[21]\right) \frac{\langle 13\rangle^{2}}{m_{C}^{2}} .
$$

that behaves as expected in the holomorphic soft limit.

In this case we will not consider the limit in which $k_{3} \rightarrow 0$ since the three-point amplitude $\mathcal{A}\left(1^{-}, 2^{+}, C_{0}\right)=0$ vanishes.

\section{$5.3 \mathcal{A}\left(A^{-}, A^{+}, A^{+}, H^{++}\right)$}

Consider finally the amplitude

$$
\mathcal{A}\left(1^{-}, 2^{+}, 3^{+}, H^{++}\right)=\mathcal{B}\left(1-\alpha^{\prime} s, 1-\alpha^{\prime} t\right) \frac{\langle 14\rangle^{4}[13]}{m_{H}\langle 12\rangle\langle 23\rangle\langle 45\rangle^{2}} .
$$

Expanding for $u_{2} \rightarrow 0$, one finds

$$
\mathcal{A}\left(1^{-}, 2^{+}, 3^{+}, H^{++}\right)=\frac{1}{\delta^{2}} \frac{\langle 14\rangle^{4}}{\langle 12\rangle\langle 23\rangle\langle 31\rangle\langle 45\rangle^{2}} m_{H}(\delta)=\frac{1}{\delta^{2}} \frac{\langle 14\rangle^{4}[45]^{2}}{\langle 12\rangle\langle 23\rangle\langle 31\rangle m_{H}^{3}(\delta)} .
$$

Using

$$
\langle 14\rangle[45]=\langle 13\rangle[35]+\delta\langle 12\rangle[25],
$$

we have

$$
\mathcal{A}\left(1^{-}, 2^{+}, 3^{+}, H^{++}\right)=\frac{1}{\delta^{2}} \frac{\langle 14\rangle^{2}[35]^{2}\langle 31\rangle}{\langle 12\rangle\langle 23\rangle m_{H}(\delta)^{3}}\left(1+2 \delta \frac{\langle 12\rangle[25]}{\langle 13\rangle[35]}-\frac{3}{2} \delta\left(\frac{\langle 12\rangle[12]}{\langle 13\rangle[13]}+\frac{\langle 23\rangle[23]}{\langle 13\rangle[13]}\right)\right) .
$$

At this stage the soft limit appears straightforward. The leading term reads

$$
\mathcal{A}^{(-2)}\left(1^{-}, 2^{+}, 3^{+}, H^{++}\right)=\frac{\langle 31\rangle}{\langle 12\rangle\langle 23\rangle} \frac{\langle 14\rangle^{2}[35]^{2}}{m_{H}^{3}}
$$

Using the expressions for the two derivatives

$$
\begin{aligned}
\frac{d}{d \bar{u}_{1}} \mathcal{A}_{3}\left(1^{-}, 3^{+}, H^{++}\right) & =-\frac{3}{2 m_{H}^{5}}\langle 14\rangle^{2}[35]^{2}\langle 13\rangle \bar{u}_{3} \\
\frac{d}{d \bar{u}_{3}} \mathcal{A}_{3}\left(1^{-}, 3^{+}, H^{++}\right) & =\frac{2}{m_{H}^{3}}\langle 14\rangle^{2}[35] \bar{u}_{5}+\frac{3}{2 m_{H}^{5}}\langle 14\rangle^{2}[35]^{2}\langle 13\rangle \bar{u}_{1}
\end{aligned}
$$

into the soft sub-leading term

$$
\mathcal{A}^{(-1)}\left(1^{-}, 2^{+}, 3^{+}, H^{++}\right)=\frac{\langle 31\rangle}{\langle 12\rangle\langle 23\rangle}\left(\frac{\langle 23\rangle}{\langle 13\rangle} \bar{u}_{2} \frac{d}{d \bar{u}_{1}}+\frac{\langle 21\rangle}{\langle 31\rangle} \bar{u}_{2} \frac{d}{d \bar{u}_{3}}\right) \frac{\langle 14\rangle^{2}[35]^{2}}{m_{H}^{3}}
$$

we reproduce exactly eq. (5.27).

Let's consider the limit in which $k_{3} \rightarrow 0$. Expanding the amplitude in eq. (5.24) up to the order $\delta^{-1}$ we find

$$
\mathcal{A}\left(1^{-}, 2^{+}, 3^{+}, H^{++}\right)=\frac{1}{\delta^{2}} \frac{\langle 14\rangle^{2}\langle 12\rangle[13][25]^{2}}{2 k_{3} p_{4}\langle 23\rangle m_{0}^{3}}\left(1-\frac{3}{2} \delta \frac{\langle 13\rangle[13]+\langle 23\rangle[23]}{\langle 12\rangle[12]}+2 \delta \frac{\langle 13\rangle[35]}{\langle 12\rangle[25]}\right) .
$$


Using the leading order soft operator we derived in section 5.1, we find that

$$
\mathcal{A}^{(-2)}\left(1^{-}, 2^{+}, 3^{+}, H^{++}\right)=\frac{1}{\delta^{2}} \frac{\langle 12\rangle[13]}{\langle 23\rangle 2 k_{3} p_{4}} \mathcal{A}\left(1^{-}, 2^{+}, H^{++}\right)=\frac{1}{\delta^{2}} \frac{\langle 14\rangle^{2}\langle 12\rangle[13][25]^{2}}{2 k_{3} p_{4}\langle 23\rangle m_{0}^{3}},
$$

using

$$
\mathcal{A}\left(1^{-}, 2^{+}, H^{++}\right)=\frac{\langle 14\rangle^{2}[25]^{2}}{m_{0}^{3}} .
$$

The sub-leading soft behavior of the amplitude is determined by

$$
\begin{aligned}
\frac{[32]}{2 k_{2} k_{3}} \bar{u}_{3} \frac{d}{d \bar{u}_{2}} \frac{\langle 14\rangle^{2}[25]^{2}}{m_{0}^{3}} & =\frac{3}{2 \delta} \frac{\langle 14\rangle^{2}[13][25]^{2}}{\langle 23\rangle m_{0}^{3}}, \\
\frac{1}{2 k_{3} p_{4}}\left([34] \bar{u}_{3} \frac{d}{d \bar{u}_{4}}+[35] \bar{u}_{3} \frac{d}{d \bar{u}_{5}}\right) \frac{\langle 14\rangle^{2}[25]^{2}}{m_{0}^{3}} & =2 \frac{\langle 14\rangle^{2}[23][35]}{2 k_{3} p_{4} m_{0}^{3}} .
\end{aligned}
$$

Following the same algebraic manipulations as in section 5.1 it can be shown that these two terms reproduce the sub-leading soft term of the expansion in eq. (5.24).

\section{Conclusions}

We have computed several open bosonic and super- string scattering amplitudes on the disk with massive and tachyonic external states in critical dimension as well as in $D=4$ (for the superstring, using the spinor helicity basis).

We have then checked their universal behaviour when massless gluons go soft, despite the presence of higher derivative couplings, and offered a general argument to this effect based on world-sheet OPE. We have also checked consistency of the factorisation on the first massive pole of the MSS formula obtained in [35, 36] relating open superstring amplitudes on the disk to tree-level SYM amplitudes.

We have only briefly considered closed strings. For gravitons, even in the presence of massive external legs, one would expect a universal soft behaviour up to sub-sub-leading order $\left(\delta^{+1}\right)[8-10,47-49]$

$$
\begin{aligned}
& \mathcal{M}_{n}(1,2, \ldots, s, \ldots, n)= \\
& \sum_{i \neq s}\left[\frac{k_{i} \cdot h_{s} \cdot k_{i}}{k_{s} \cdot k_{i}}+\frac{2 k_{i} \cdot h_{s} \cdot J_{i} k_{s}}{k_{s} \cdot k_{i}}+\frac{k_{s} \cdot J_{i} \cdot h_{s} \cdot J_{i} \cdot k_{s}}{k_{s} \cdot k_{i}}\right] \mathcal{M}_{n-1}(1,2, \ldots \hat{s} \ldots, n)+\mathcal{O}\left(\delta^{2}\right)
\end{aligned}
$$

This should hold true at tree-level and with the understanding that interactions be governed by minimal couplings. While in closed Type II superstrings on the sphere the soft limit of amplitudes with massless states is the same as in gravity at tree level, for bosonic strings - and in fact for the heterotic string, too - the presence of a $\phi R^{2}$ vertex with the dilaton spoils the universal behave even at leading order, in that a soft graviton attached to a hard graviton can produce a hard dilaton thus producing a mixed amplitude ${ }^{9}$

\footnotetext{
${ }^{9}$ As suggested in [46], one may be tempted to propose a generalisation of the soft theorem whereby dilatons and gravitons are 'unified' into a gravi-dilaton with symmetric transverse but non-traceless polarisation tensor $e_{\mu \nu}=h_{\mu \nu}+\phi_{\mu \nu}$ with $\phi_{\mu \nu}=\eta_{\mu \nu}-k_{\mu} \bar{k}_{\nu}-k_{\nu} \bar{k}_{\mu}$ and $\bar{k}^{2}=0 \bar{k} k=1$. Yet for the Kalb-Ramond anti-symmetric tensor $b_{\mu \nu}$ which is odd under world-sheet parity $\Omega$, one expects a vanishing behaviour at leading order [14].
} 
Using KLT relations [41] one can efficiently compute closed amplitudes with massive external states as 'squares' of open string amplitudes with massive external states, like the ones we have considered in the present investigation. We plan to carry out this analysis in simple cases and study the soft behaviour at tree level confirming universality, respectively lack of it, in the case of the closed superstring (both Type IIA and Type IIB), respectively in the case of the bosonic or heterotic string due to the presence of the $\phi R^{2}$ terms [52]. We hope to shed further light on the soft behaviour of the Kalb-Ramond field, the dilaton [50] and the other moduli fields [51]. It would also be interesting to investigate the soft behaviour of loop amplitudes and to test the validity of the new proposal $[53,54]$ of getting the graviton from the collinear limit of two gluons beyond tree level and in the presence of massive external states.

\section{Acknowledgments}

We would like to thank P. Di Vecchia, C. Mafra, R. Marotta, M. Mojaza, O. Schlotterer, S. Stieberger, C-K Wen for discussions.

\section{A Open bosonic string 3-point amplitudes}

For the sake of completeness we summarize all the possible three point functions involving open bosonic string states up to the first massive level, eqs. (2.1), (2.2), (2.3). Kinematics of three point on-shell amplitudes is fixed in terms of the masses of the particles involved in the process. This property will be used repeatedly and stressed wherever necessary. In the following formulas a factor $(2 \pi)^{D} \delta^{D}\left(\sum_{i} p_{i}\right)$, with $D \leq 26$, resulting from integration over the zero mode of the coordinate fields $X^{\mu}$, is always understood. We will also drop a factor of $g_{s}\left(\alpha^{\prime}\right)^{(D / 2-3) / 2}$, which is $g_{s}\left(\alpha^{\prime}\right)^{5}$ for the bosonic string in critical dimension, but, following the discussion in section 2.2 , we will explicitly include the relevant Chan-Paton factors $f_{a b c}$ or $d_{a b c}$ that make the full 'amplitude' Bose symmetric.

\section{- $T T T$ vertex}

$$
\begin{aligned}
\mathcal{A}\left(T_{1}, T_{2}, T_{3}\right) & =d_{a b c}\left\langle c e^{i p_{1} X}\left(z_{1}\right) c e^{i p_{2} X}\left(z_{2}\right) c e^{i p_{3} X}\left(z_{3}\right)\right\rangle \\
& =d_{a b c} z_{12} z_{13} z_{23} z_{12}^{2 \alpha^{\prime} p_{1} p_{2}} z_{13}^{2 \alpha^{\prime} p_{1} p_{3}} z_{23}^{2 \alpha^{\prime} p_{2} p_{3}}=d_{a b c}
\end{aligned}
$$

where we used the identity $\left(p_{i}+p_{j}\right)^{2}=-2 m_{T}^{2}+2 p_{i} p_{j}=-m_{T}^{2}$, so that $2 \alpha^{\prime} p_{i} p_{j}=m_{T}^{2}=-\frac{1}{\alpha^{\prime}}$ for all $i, j$. The symbol $z_{i j}$ stands for $z_{i}-z_{j}$. In order to simplify the notation, from now on we will introduce the notation

$$
P_{i}^{\mu}=\sum_{j \neq i} \frac{p_{j}^{\mu}}{z_{j i}} .
$$

In general, $P_{i}$ is contracted always with the $i$-th polarization vector/tensor. Exploiting 'transversality' i.e. $p_{i}^{\mu} t_{\mu \ldots}^{i}=0$, we will always replace the sum in eq. (A.2) with:

$$
P_{1}=\frac{p_{23}}{2} \frac{z_{23}}{z_{12} z_{13}}, \quad P_{2}=\frac{p_{31}}{2} \frac{z_{13}}{z_{12} z_{23}}, \quad P_{3}=\frac{p_{12}}{2} \frac{z_{12}}{z_{13} z_{23}} .
$$




\section{- $T T A$ vertex}

$$
\begin{aligned}
\mathcal{A}\left(A_{1}, T_{2}, T_{3}\right) & =\frac{1}{\sqrt{2 \alpha^{\prime}}} f_{a b c}\left\langle c a_{1 \mu} i \partial X^{\mu} e^{i k_{1} X}\left(z_{1}\right) c e^{i p_{2} X}\left(z_{2}\right) c e^{i p_{3} X}\left(z_{3}\right)\right\rangle \\
& =\sqrt{2 \alpha^{\prime}} f_{a b c} z_{12} z_{13} z_{23} a_{1 \mu} P_{1}^{\mu} \prod_{i<j} z_{i j}^{2 \alpha^{\prime} p_{i} p_{j}}=\sqrt{2 \alpha^{\prime}} f_{a b c} \frac{1}{2} a_{1} p_{23} .
\end{aligned}
$$

\section{- $T A A$ vertex}

$$
\begin{aligned}
\mathcal{A}\left(A_{1}, A_{2}, T_{3}\right) & =\frac{1}{2 \alpha^{\prime}} d_{a b c}\left\langle c a_{1} i \partial X e^{i k_{1} X}\left(z_{1}\right) c a_{2} i \partial X e^{i k_{2} X}\left(z_{2}\right) c e^{i p_{3} X}\left(z_{3}\right)\right\rangle \\
& =d_{a b c}\left(2 \alpha^{\prime} a_{1} \frac{p_{23}}{2} a_{2} \frac{p_{31}}{2}+a_{1} a_{2}\right) .
\end{aligned}
$$

The amplitude can be rewritten in a manifestly gauge invariant form:

$$
\mathcal{A}\left(A_{1}, A_{2}, T_{3}\right)=2 \alpha^{\prime} d_{a b c}\left(a_{1} \frac{p_{23}}{2} a_{2} \frac{p_{31}}{2}+a_{1} a_{2} k_{1} k_{2}\right)=2 \alpha^{\prime} d_{a b c} \frac{1}{2} f_{1 \mu \nu} f_{2}^{\nu \mu} .
$$

\section{- $A A A$ vertex}

$$
\begin{aligned}
\mathcal{A}\left(A_{1}, A_{2}, A_{3}\right) & =\frac{1}{\left(2 \alpha^{\prime}\right)^{3 / 2}} f_{a b c}\left\langle c a_{1} i \partial X e^{i k_{1} X}\left(z_{1}\right) c a_{2} i \partial X e^{i k_{2} X}\left(z_{2}\right) c a_{3} i \partial X e^{i k_{3} X}\left(z_{3}\right)\right\rangle \\
& =\sqrt{2 \alpha^{\prime}} f_{a b c}\left(a_{1} a_{2} a_{3} \frac{k_{12}}{2}+a_{1} a_{3} a_{2} \frac{k_{31}}{2}+a_{2} a_{3} a_{1} \frac{k_{23}}{2}+2 \alpha^{\prime} a_{1} \frac{k_{23}}{2} a_{2} \frac{k_{31}}{2} a_{3} \frac{k_{12}}{2}\right) .
\end{aligned}
$$

\section{- $T A H$ vertex}

$$
\begin{aligned}
\mathcal{A}\left(A_{1}, T_{2}, \mathcal{H}_{3}\right) & =\frac{1}{\left(2 \alpha^{\prime}\right)^{3 / 2}} f_{a b c}\left\langle c a_{1} i \partial X e^{i k_{1} X}\left(z_{1}\right) c e^{i p_{2} X}\left(z_{2}\right) c i \partial X H_{3} i \partial X e^{i p_{3} X}\left(z_{3}\right)\right\rangle \\
& =\sqrt{2 \alpha^{\prime}} f_{a b c}\left(2 a_{1} H_{3} \frac{p_{12}}{2}+2 \alpha^{\prime} a_{1} \frac{p_{23}}{2} \frac{p_{12}}{2} H_{3} \frac{p_{12}}{2}\right) .
\end{aligned}
$$

\section{- $A A H$ vertex}

$$
\begin{aligned}
\mathcal{A}\left(A_{1}, A_{2}, H_{3}\right)= & \frac{d_{a b c}}{\left(2 \alpha^{\prime}\right)^{2}}\left\langle c a_{1} i \partial X e^{i k_{1} X}\left(z_{1}\right) c a_{2} i \partial X e^{i k_{2} X}\left(z_{2}\right) c i \partial X H_{3} i \partial X e^{i p_{3} X}\left(z_{3}\right)\right\rangle \\
= & d_{a b c}\left(2 a_{1} H_{3} a_{2}+2 \alpha^{\prime}\left(2 a_{1} \frac{p_{23}}{2} a_{2} H_{3} \frac{p_{12}}{2}+2 a_{2} \frac{p_{31}}{2} a_{1} H_{3} \frac{p_{12}}{2}+a_{1} a_{2} \frac{p_{12}}{2} H_{3} \frac{p_{12}}{2}\right)\right. \\
& \left.-\left(2 \alpha^{\prime}\right)^{2} a_{1} \frac{p_{23}}{2} a_{2} \frac{p_{13}}{2} \frac{p_{12}}{2} H_{3} \frac{p_{12}}{2}\right) .
\end{aligned}
$$

One can rewrite the above amplitude in the manifestly gauge invariant form

$$
\mathcal{A}\left(A_{1}, A_{2}, H_{3}\right)=2 \alpha^{\prime} d_{a b c}\left(2 \operatorname{tr}\left(f_{1} H_{3} f_{2}\right)-\alpha^{\prime} \operatorname{tr}\left(f_{1} f_{2}\right) k_{1} H_{3} k_{2}\right) .
$$

\section{- $A H H$ vertex}

$$
\begin{aligned}
\mathcal{A}\left(A_{1}, H_{2}, H_{3}\right)= & \frac{f_{a b c}}{\left(2 \alpha^{\prime}\right)^{5 / 2}}\left\langle c a_{1} i \partial X e^{i k_{1} X}\left(z_{1}\right) c i \partial X H_{2} i \partial X e^{i p_{2} X}\left(z_{2}\right) c i \partial X H_{3} i \partial X e^{i p_{3} X}\left(z_{3}\right)\right\rangle \\
= & \sqrt{2 \alpha^{\prime}} f_{a b c}\left(2 a_{1} \frac{p_{23}}{2} \operatorname{tr}\left(H_{2} H_{3}\right)-4 \operatorname{tr}\left(f_{1} H_{2} H_{3}\right)+4 \alpha^{\prime} \frac{p_{31}}{2} H_{2} f_{1} H_{3} \frac{p_{12}}{2}\right. \\
& \left.+8 \alpha^{\prime} a_{1} \frac{p_{23}}{2} \frac{p_{31}}{2} H_{2} H_{3} \frac{p_{12}}{2}+\left(2 \alpha^{\prime}\right)^{2} a_{1} \frac{p_{23}}{2} \frac{p_{31}}{2} H_{2} \frac{p_{31}}{2} \frac{p_{12}}{2} H_{3} \frac{p_{12}}{2}\right) .
\end{aligned}
$$


- $T H H$ vertex

$$
\begin{aligned}
\mathcal{A}\left(T_{1}, H_{2}, H_{3}\right) & =\frac{1}{\left(2 \alpha^{\prime}\right)^{2}} d_{a b c}\left\langle c e^{i p_{1} X}\left(z_{1}\right) c i \partial X H_{2} i \partial X e^{i p_{2} X}\left(z_{2}\right) c i \partial X H_{3} i \partial X e^{i p_{3} X}\left(z_{3}\right)\right\rangle \\
& =d_{a b c}\left(2 \operatorname{tr}\left(H_{2} H_{3}\right)+4\left(2 \alpha^{\prime}\right) \frac{p_{31}}{2} H_{2} H_{3} \frac{p_{12}}{2}+\left(2 \alpha^{\prime}\right)^{2} \frac{p_{31}}{2} H_{2} \frac{p_{31}}{2} \frac{p_{12}}{2} H_{3} \frac{p_{12}}{2}\right) .
\end{aligned}
$$

\section{- $T T H$ vertex}

$$
\begin{aligned}
\mathcal{A}\left(T_{1}, T_{2}, H_{3}\right) & =\frac{1}{2 \alpha^{\prime}} d_{a b c}\left\langle c e^{i p_{1} X}\left(z_{1}\right) c e^{i p_{2} X}\left(z_{2}\right) c i \partial X H_{3} i \partial X e^{i p_{3} X}\left(z_{3}\right)\right\rangle \\
& =d_{a b c} z_{12} z_{13} z_{23} \prod_{i<j} z_{i j}^{2 \alpha^{\prime} p_{i} p_{j}} 2 \alpha^{\prime} P_{3} H_{3} P_{3}=2 \alpha^{\prime} d_{a b c} \frac{p_{12}}{2} H_{3} \frac{p_{12}}{2} .
\end{aligned}
$$

\section{- $H H H$ vertex}

$$
\begin{aligned}
& \mathcal{A}\left(H_{1}, H_{2}, H_{3}\right) \\
&=\frac{d_{a b c}}{\left(2 \alpha^{\prime}\right)^{3}}\left\langle c i \partial X H_{1} i \partial X e^{i p_{1} X}\left(z_{1}\right) c i \partial X H_{2} i \partial X e^{i p_{2} X}\left(z_{2}\right) c i \partial X H_{3} i \partial X e^{i p_{3} X}\left(z_{3}\right)\right\rangle \\
&= d_{a b c}\left(8 \operatorname{tr}\left(H_{1} H_{2} H_{3}\right)+2 \alpha^{\prime}\left(\operatorname{tr}\left(H_{1} H_{2}\right) \frac{p_{12}}{2} H_{3} \frac{p_{12}}{2}+\operatorname{tr}\left(H_{1} H_{3}\right) \frac{p_{31}}{2} H_{2} \frac{p_{31}}{2}\right.\right. \\
&\left.+\operatorname{tr}\left(H_{2} H_{3}\right) \frac{p_{23}}{2} H_{1} \frac{p_{23}}{2}+8 \frac{p_{23}}{2} H_{1} H_{2} H_{3} \frac{p_{12}}{2}+8 \frac{p_{23}}{2} H_{1} H_{3} H_{2} \frac{p_{31}}{2}+8 \frac{p_{31}}{2} H_{2} H_{1} H_{3} \frac{p_{12}}{2}\right) \\
&+\left(2 \alpha^{\prime}\right)^{2}\left(\frac{p_{23}}{2} H_{1} H_{2} \frac{p_{31}}{2} \frac{p_{12}}{2} H_{3} \frac{p_{12}}{2}+\frac{p_{23}}{2} H_{1} H_{3} \frac{p_{12}}{2} \frac{p_{31}}{2} H_{2} \frac{p_{31}}{2}+\frac{p_{31}}{2} H_{1} H_{3} \frac{p_{12}}{2} \frac{p_{23}}{2} H_{1} \frac{p_{23}}{2}\right) \\
&\left.+\left(2 \alpha^{\prime}\right)^{3} \frac{p_{23}}{2} H_{1} \frac{p_{23}}{2} \frac{p_{31}}{2} H_{2} \frac{p_{31}}{2} \frac{p_{12}}{2} H_{3} \frac{p_{12}}{2}\right) .
\end{aligned}
$$

\section{B Open bosonic string four-point amplitudes}

In this appendix we sketch the computation of the open bosonic string amplitudes involving massive and tachyonic states. For simplicity we consider color-ordered amplitudes. Complete amplitudes arise after multiplying by the relevant Chan-Paton factors $\operatorname{tr}\left(t^{1} t^{2} t^{3} t^{4}\right)$ and summing over non-cyclic permutations. In fact, exploting 'twist symmetry' i.e. $\Omega$ invariance, one can reduce the sum to three terms (instead of six). Exploiting conformal invariance we choose to fix $z_{1} \rightarrow \infty, z_{2}=1, z=\frac{z_{12} z_{34}}{z_{13} z_{24}}$ and $z_{4}=0$. A factor $g_{s, a p}^{2}\left(\alpha^{\prime}\right)^{(D / 2-4) / 2}(2 \pi)^{D} \delta^{D}\left(\sum_{i} p_{i}\right)$ is always understood.

\section{- Veneziano amplitude (TTTT)}

$$
\begin{aligned}
& \mathcal{A}\left(T_{1}, T_{2}, T_{3}, T_{4}\right)=\left\langle c e^{i p_{1} X}\left(z_{1}\right) c e^{i p_{2} X}\left(z_{2}\right) \int d z_{3} e^{i p_{3} X}\left(z_{3}\right) c e^{i p_{4} X}\left(z_{4}\right)\right\rangle \\
& =z_{12} z_{14} z_{24} \int d z_{3} \prod_{i<j} z_{i j}^{2 \alpha^{\prime} p_{i} p_{j}}=\int_{0}^{1} d z(1-z)^{2 \alpha^{\prime} p_{2} p_{3}} z^{2 \alpha^{\prime} p_{3} p_{4}}=B\left(1+2 \alpha^{\prime} p_{2} p_{3}, 1+2 \alpha^{\prime} p_{3} p_{4}\right) .
\end{aligned}
$$

Introducing the Mandelstam variables $\left(p_{1}+p_{2}\right)^{2}=\left(p_{3}+p_{4}\right)^{2}=-s,\left(p_{2}+p_{3}\right)^{2}=\left(p_{1}+p_{4}\right)^{2}=-t$, $\left(p_{1}+p_{3}\right)^{2}=\left(p_{2}+p_{4}\right)^{2}=-u$, we can rewrite the Veneziano amplitude as

$$
\mathcal{A}\left(T_{1}, T_{2}, T_{3}, T_{4}\right)=\frac{\Gamma\left(-1-\alpha^{\prime} s\right) \Gamma\left(-1-\alpha^{\prime} t\right)}{\Gamma\left(-2-\alpha^{\prime}(s+t)\right)} .
$$




\section{- ATTT amplitude}

$$
\begin{aligned}
\mathcal{A}\left(A_{1}, T_{2}, T_{3}, T_{4}\right) & =\frac{1}{\sqrt{2 \alpha^{\prime}}}\left\langle c a_{1} i \partial X e^{i k_{1} X}\left(z_{1}\right) c e^{i p_{2} X}\left(z_{2}\right) \int d z_{3} e^{i p_{3} X}\left(z_{3}\right) c e^{i p_{4} X}\left(z_{4}\right)\right\rangle \\
& =\frac{1}{\sqrt{2 \alpha^{\prime}}}\left(\frac{a_{1} p_{2}}{k_{1} p_{2}}-\frac{a_{1} p_{4}}{k_{1} p_{4}}\right) \frac{\Gamma\left(1+2 \alpha^{\prime} k_{1} p_{2}\right) \Gamma\left(1+2 \alpha^{\prime} k_{1} p_{4}\right)}{\Gamma\left(1-2 \alpha^{\prime} k_{1} p_{3}\right)} .
\end{aligned}
$$

\section{- AATT amplitude}

$$
\begin{aligned}
\mathcal{A}( & \left.A_{1}, A_{2}, T_{3}, T_{4}\right)=\frac{1}{2 \alpha^{\prime}}\left\langle c a_{1} i \partial X e^{i k_{1} X}\left(z_{1}\right) c a_{2} i \partial X e^{i k_{2} X}\left(z_{2}\right) \int d z_{3} e^{i p_{3} X}\left(z_{3}\right) c e^{i p_{4} X}\left(z_{4}\right)\right\rangle \\
= & \left(a_{1} a_{2}-2 \alpha^{\prime}\left(a_{1} p_{3} a_{2} p_{3}+a_{1} p_{4} a_{2} p_{4}\right)+a_{1} p_{3} a_{2} p_{4} \frac{1+2 \alpha^{\prime} k_{1} p_{4}}{k_{1} p_{3}}+a_{1} p_{4} a_{2} p_{3} \frac{1+2 \alpha^{\prime} k_{1} p_{3}}{k_{1} p_{4}}\right) \\
& B\left(1+2 \alpha^{\prime} k_{1} p_{4},-1+2 \alpha^{\prime} k_{1} k_{2}\right) .
\end{aligned}
$$

\section{- $A A A T$ amplitude}

$$
\begin{aligned}
& \mathcal{A}\left(A_{1}, A_{2}, T_{3}, A_{4}\right)=\int d z_{3}\left\langle c a_{2} i \partial X e^{i p_{1} X}\left(z_{1}\right) c a_{1} i \partial X e^{i p_{2} X}\left(z_{2}\right) e^{i p_{3} X}\left(z_{3}\right) c a_{4} i \partial X e^{i p_{4} x}\left(z_{4}\right)\right\rangle \\
& =\frac{\Gamma\left(1+2 \alpha^{\prime} p_{1} p_{2}\right) \Gamma\left(1+2 \alpha^{\prime} p_{1} p_{4}\right)}{\Gamma\left(1-2 \alpha^{\prime} p_{1} p_{3}\right)} \frac{p_{1} p_{3}}{2 \alpha^{\prime} p_{1} p_{2} p_{1} p_{4}}\left\{-a_{1} a_{2} a_{4} p_{1} \frac{1+2 \alpha^{\prime} p_{1} p_{3}}{2 \alpha^{\prime} p_{3} p_{4}}+a_{1} a_{2} a_{4} p_{2} \frac{1+2 \alpha^{\prime} p_{2} p_{3}}{2 \alpha^{\prime} p_{3} p_{4}}\right. \\
& +a_{1} a_{4} a_{2} p_{1} \frac{1+2 \alpha^{\prime} p_{1} p_{3}}{2 \alpha^{\prime} p_{2} p_{3}}-a_{1} a_{4} a_{2} p_{4} \frac{1+2 \alpha^{\prime} p_{3} p_{4}}{2 \alpha^{\prime} p_{2} p_{3}}-a_{2} a_{4} a_{1} p_{2} \frac{1+2 \alpha^{\prime} p_{2} p_{3}}{2 \alpha^{\prime} p_{1} p_{3}}+a_{2} a_{4} a_{1} p_{4} \frac{1+2 \alpha^{\prime} p_{3} p_{4}}{2 \alpha^{\prime} p_{1} p_{3}} \\
& +2 \alpha^{\prime}\left(a_{1} p_{4} a_{2} p_{1} a_{4} p_{2}-a_{1} p_{2} a_{2} p_{4} a_{4} p_{1}+a_{1} p_{2} a_{2} p_{1} a_{4} p_{1} \frac{1+2 \alpha^{\prime} p_{1} p_{3}}{2 \alpha^{\prime} p_{3} p_{4}}-a_{1} p_{4} a_{2} p_{1} a_{4} p_{1} \frac{1+2 \alpha^{\prime} p_{1} p_{3}}{2 \alpha^{\prime} p_{2} p_{3}}\right. \\
& -a_{1} p_{4} a_{2} p_{4} a_{4} p_{2} \frac{1+2 \alpha^{\prime} p_{3} p_{4}}{2 \alpha^{\prime} p_{1} p_{3}}+a_{1} p_{2} a_{2} p_{4} a_{4} p_{2} \frac{1+2 \alpha^{\prime} p_{2} p_{3}}{2 \alpha^{\prime} p_{1} p_{3}}+a_{1} p_{4} a_{2} p_{4} a_{4} p_{1} \frac{1+2 \alpha^{\prime} p_{3} p_{4}}{2 \alpha^{\prime} p_{2} p_{3}} \\
& \left.\left.-a_{1} p_{2} a_{2} p_{1} a_{4} p_{2} \frac{1+2 \alpha^{\prime} p_{2} p_{3}}{2 \alpha^{\prime} p_{3} p_{4}}\right)\right\} .
\end{aligned}
$$

\section{- $T T A H$ amplitude}

$$
\begin{aligned}
\mathcal{A} & \left(T_{1}, T_{2}, A_{3}, H_{4}\right)=\frac{1}{\left(2 \alpha^{\prime}\right)^{\frac{3}{2}}}\left\langle c e^{i p_{1} X}\left(z_{1}\right) c e^{i p_{2} X}\left(z_{2}\right) \int d z_{3} a i \partial X e^{i k X}\left(z_{3}\right) c i \partial X H i \partial X e^{i p_{4} X}\left(z_{4}\right)\right\rangle \\
= & \left(-2 a_{3} H p_{2}-2 a_{3} H k_{3} \frac{1+2 \alpha^{\prime} k_{3} p_{1}}{2-2 \alpha^{\prime} k_{3} p_{4}}+2 \alpha^{\prime} a_{3} p_{4}\left(p_{2} H p_{2} \frac{1-2 \alpha^{\prime} k_{3} p_{4}}{2 \alpha^{\prime} k_{3} p_{1}}+k_{3} H k_{3} \frac{1+2 \alpha^{\prime} k_{3} p_{1}}{2-2 \alpha^{\prime} k_{3} p_{4}}+2 p_{2} H p_{3}\right)\right. \\
& \left.-2 \alpha^{\prime} a_{3} p_{2}\left(\frac{k_{3} p_{4} p_{2} H p_{2}}{2 \alpha^{\prime} p_{2} k_{3} p_{1} k_{3}}\left(1-2 \alpha^{\prime} k_{3} p_{4}\right)-p_{3} H p_{3} \frac{1+2 \alpha^{\prime} p_{1} k_{3}}{2 \alpha^{\prime} p_{2} k_{3}}-2 p_{2} H k_{3} \frac{1-2 \alpha^{\prime} k_{3} p_{4}}{2 \alpha^{\prime} p_{2} k_{3}}\right)\right) \\
& \frac{\Gamma\left(-1+2 \alpha^{\prime} k_{3} p_{4}\right) \Gamma\left(1+2 \alpha^{\prime} p_{2} k_{3}\right)}{\Gamma\left(-2 \alpha^{\prime} p_{1} k_{3}\right)} .
\end{aligned}
$$

\section{Open superstring 3-point amplitudes}

In this section we compute all the possible tri-linear couplings involving superstring states up to the first massive level in the Neveu-Schwarz sector following the same conventions as in appendix A. 
- $A A C$ vertex

$$
\begin{aligned}
& \mathcal{A}\left(A_{1}^{(0)}, A_{2}^{(-1)}, C_{3}^{(-1)}\right) \\
& =\frac{d_{a b c}}{\sqrt{2 \alpha^{\prime}}}\left\langle c\left(a_{1} i \partial X+k_{1} \psi a_{1} \psi\right) e^{i k_{1} X}\left(z_{1}\right) c e^{-\phi} a_{2} \psi e^{i k_{2} X}\left(z_{2}\right) c e^{-\phi} C_{3} \psi \psi \psi e^{i p_{3} X}\left(z_{3}\right)\right\rangle \\
& =\sqrt{2 \alpha^{\prime}} d_{a b c} \frac{z_{12} z_{13} z_{23}}{z_{23}} \prod_{i<j} z_{i j}^{2 \alpha^{\prime} p_{i} p_{j}} 6 \frac{a_{2}^{\mu} C_{3 \mu \nu \rho} a_{1}^{\nu} k_{1}^{\rho}}{z_{13}^{2} z_{23}}=6 \sqrt{2 \alpha^{\prime}} d_{a b c} a_{2}^{\mu} C_{3 \mu \nu \rho} a_{1}^{\nu} \frac{k_{12}^{\rho}}{2} .
\end{aligned}
$$

\section{- $A A H$ vertex}

$$
\begin{aligned}
& \mathcal{A}\left(A_{1}^{(0)}, A_{2}^{(-1)}, H_{3}^{(-1)}\right) \\
& =\frac{d_{a b c}}{2 \alpha^{\prime}}\left\langle c\left(a_{1} i \partial X+k_{1} \psi a_{1} \psi\right) e^{i k_{1} X}\left(z_{1}\right) c e^{-\phi} a_{2} \psi e^{i k_{2} X}\left(z_{2}\right) c e^{-\phi} i \partial X H_{3} \psi e^{i p_{3} X}\left(z_{3}\right)\right\rangle \\
& =-2 \alpha^{\prime} d_{a b c} \operatorname{tr}\left(f_{1} H_{3} f_{2}\right) .
\end{aligned}
$$

\section{- $A H C$ vertex}

$$
\begin{aligned}
& \mathcal{A}\left(A_{1}^{(-1)}, H_{2}^{(0)}, C_{3}^{(0)}\right) \\
& =\frac{f_{a b c}}{2 \alpha^{\prime}}\left\langle c\left(a_{1} i \partial X+k_{1} \psi a_{1} \psi\right) e^{i k_{1} X}\left(z_{1}\right) c e^{-\phi} i \partial X H_{2} \psi e^{i p_{2} X}\left(z_{2}\right) c e^{-\phi} C_{3} \psi \psi \psi e^{i p_{3} X}\left(z_{3}\right)\right\rangle \\
& =2 \alpha^{\prime} f_{a b c} \frac{z_{12} z_{13} z_{23}}{z_{23}} \prod_{i<j} z_{i j}^{2 \alpha^{\prime} p_{i} p_{j}} 6 P_{2}^{\rho} H_{2 \rho \sigma} C_{3 \mu \nu \lambda} \frac{\eta^{\mu \sigma} a_{1}^{\nu} k_{1}^{\lambda}}{z_{23} z_{13}^{2}}=f_{a b c} 12 \alpha^{\prime} \frac{p_{31}}{2} H_{2} C_{3} a_{1} \frac{p_{12}}{2} .
\end{aligned}
$$

\section{- $A H H$ vertex}

$$
\begin{aligned}
& \mathcal{A}\left(A_{1}^{(0)}, H_{2}^{(-1)}, H_{3}^{(-1)}\right) \\
& =\frac{f_{a b c}}{\left(2 \alpha^{\prime}\right)^{3 / 2}}\left\langle c\left(a_{1} i \partial X+k_{1} \psi a_{1} \psi\right) e^{i k_{1} X}\left(z_{1}\right) c e^{-\phi} i \partial X H_{2} \psi e^{i p_{2} X}\left(z_{2}\right) c e^{-\phi} i \partial X H_{3} \psi e^{i p_{3} X}\left(z_{3}\right)\right\rangle \\
& =\sqrt{2 \alpha^{\prime}} f_{a b c}\left(2 \operatorname{tr}\left(f_{1} H_{2} H_{3}\right)+a_{1} \frac{p_{23}}{2} \operatorname{tr}\left(H_{2} H_{3}\right)+2 \alpha^{\prime}\left(\frac{p_{31}}{2} H_{2} f_{1} H_{3} \frac{p_{12}}{2}+a_{1} \frac{p_{23}}{2} \frac{p_{31}}{2} H_{2} H_{3} \frac{p_{12}}{2}\right)\right) .
\end{aligned}
$$

\section{- $A C C$ vertex}

$$
\begin{aligned}
& \mathcal{A}\left(A_{1}^{(0)}, C_{2}^{(-1)}, C_{3}^{(-1)}\right) \\
& =\frac{f_{a b c}}{\sqrt{2 \alpha^{\prime}}}\left\langle c\left(a_{1} i \partial X+k_{1} \psi a_{1} \psi\right) e^{i k_{1} X}\left(z_{1}\right) c e^{-\phi} C_{2} \psi \psi \psi e^{i p_{2} X}\left(z_{2}\right) c e^{-\phi} C_{3} \psi \psi \psi e^{i p_{3} X}\left(z_{3}\right)\right\rangle \\
& =\sqrt{2 \alpha^{\prime}} f_{a b c} 6\left(a_{1} \frac{p_{23}}{2} \operatorname{tr}\left(C_{2} C_{3}\right)-3 \operatorname{tr}\left(f_{1} C_{2} C_{3}\right)\right) .
\end{aligned}
$$

\section{- $A A A$ vertex}

$$
\begin{aligned}
& \mathcal{A}\left(A_{1}^{(0)}, A_{2}^{(-1)}, A_{3}^{(-1)}\right) \\
& =\frac{f_{a b c}}{\sqrt{2 \alpha^{\prime}}}\left\langle c\left(z_{1}\right)\left(a_{1} i \partial X+k_{1} \psi a_{1} \psi\right) e^{i k_{1} X}\left(z_{1}\right) c e^{-\varphi} a_{2} \psi e^{i k_{2} X}\left(z_{2}\right) c e^{-\varphi} a_{3} \psi e^{i k_{3} X}\left(z_{3}\right)\right\rangle \\
& =\sqrt{2 \alpha^{\prime}} f_{a b c}\left(a_{1} \frac{k_{23}}{2} a_{2} a_{3}+a_{2} \frac{k_{31}}{2} a_{1} a_{3}+a_{3} \frac{k_{12}}{2} a_{1} a_{2}\right) .
\end{aligned}
$$




\section{- $H H H$ vertex}

$$
\begin{aligned}
& \mathcal{A}\left(H_{1}^{(0)}, H_{2}^{(-1)}, H_{3}^{(-1)}\right)=\frac{d_{a b c}}{\left(2 \alpha^{\prime}\right)^{2}}\left\langle c H_{1 \mu \nu}\left(i \partial X^{\mu} i \partial X^{\nu}+p_{1} \psi \psi^{\mu} i \partial X^{\nu}+\partial \psi^{\mu} \psi^{\nu}\right) e^{i p_{1} X}\left(z_{1}\right)\right. \\
& \left.=3 e^{-\varphi} i \partial X H_{2} \psi e^{i p_{2} X}\left(z_{2}\right) c e^{-\varphi} i \partial X H_{3} \psi e^{i p_{3} X}\left(z_{3}\right)\right\rangle \\
& =3 \operatorname{tr}\left(H_{1} H_{2} H_{3}\right)+2 \alpha^{\prime}\left(3 \frac{p_{23}}{2} H_{1} H_{2} H_{3} \frac{p_{12}}{2}+3 \frac{p_{23}}{2} H_{1} H_{3} H_{2} \frac{p_{31}}{2}+3 \frac{p_{31}}{2} H_{2} H_{1} H_{3} \frac{p_{12}}{2}\right. \\
& \left.\quad+\operatorname{tr}\left(H_{2} H_{3}\right) \frac{p_{23}}{2} H_{1} \frac{p_{23}}{2}+\operatorname{tr}\left(H_{1} H_{3}\right) \frac{p_{31}}{2} H_{2} \frac{p_{31}}{2}+\operatorname{tr}\left(H_{1} H_{2}\right) \frac{p_{12}}{2} H_{3} \frac{p_{12}}{2}\right) \\
& \quad+\left(2 \alpha^{\prime}\right)^{2}\left(\frac{p_{23}}{2} H_{1} \frac{p_{23}}{2} \frac{p_{31}}{2} H_{2} H_{3} \frac{p_{12}}{2}+\frac{p_{31}}{2} H_{2} \frac{p_{31}}{2} \frac{p_{23}}{2} H_{1} H_{3} \frac{p_{12}}{2}+\frac{p_{12}}{2} H_{3} \frac{p_{12}}{2} \frac{p_{23}}{2} H_{1} H_{2} \frac{p_{31}}{2}\right) .
\end{aligned}
$$

\section{- $C C C$ vertex}

$$
\begin{aligned}
& \mathcal{A}\left(C_{1}^{(0)}, C_{2}^{(-1)}, C_{3}^{(-1)}\right) \\
& =\frac{d_{a b c}}{\sqrt{2 \alpha^{\prime}}}\left\langle c C_{1 \mu \nu \rho}\left(i \partial X^{\mu}+p \psi \psi^{\mu}\right) \psi^{\nu} \psi^{\rho} e^{i p_{1} X}\left(z_{1}\right) c e^{-\varphi} C_{2} \psi \psi \psi e^{i p_{2} X}\left(z_{2}\right) c e^{-\varphi} \psi \psi \psi e^{i p_{3} X}\left(z_{3}\right)\right\rangle \\
& =\sqrt{2 \alpha^{\prime}} d_{a b c}\left(\frac{p_{23}^{\mu}}{2} C_{1 \mu \nu \rho} C_{2}^{\rho \sigma \lambda} C_{3 \lambda \sigma}{ }^{\nu}+\frac{p_{31}^{\mu}}{2} C_{2 \mu \nu \rho} C_{3}^{\rho \sigma \lambda} C_{1 \lambda \sigma}{ }^{\nu}+\frac{p_{12}^{\mu}}{2} C_{3 \mu \nu \rho} C_{1}^{\rho \sigma \lambda} C_{3 \lambda \sigma}{ }^{\nu}\right) .
\end{aligned}
$$

\section{- $C C H$ vertex}

$$
\begin{aligned}
& \mathcal{A}\left(C_{1}^{(0)}, C_{2}^{(-1)}, H_{3}^{(-1)}\right) \\
= & \frac{d_{a b c}}{2 \alpha^{\prime}}\left\langle c C_{1 \mu \nu \rho}\left(i \partial X^{\mu}+p \psi \psi^{\mu}\right) \psi^{\nu} \psi^{\rho} e^{i p_{1} X}\left(z_{1}\right) c e^{-\varphi} C_{2} \psi \psi \psi e^{i p_{2} X}\left(z_{2}\right) c e^{-\varphi} i \partial X H_{3} \psi e^{i p_{3} X}\left(z_{3}\right)\right\rangle \\
& \left.d_{a b c} 6 \operatorname{tr}\left(C_{1} C_{2} H_{3}\right)+2 \alpha^{\prime}\left(6 \operatorname{tr}\left(C_{1} C_{2}\right) \frac{p_{12}}{2} H_{3} \frac{p_{12}}{2}+\frac{p_{23}}{2} C_{1} C_{2} H_{3} \frac{p_{12}}{2}+6 \frac{p_{12}}{2} H_{3} C_{1} C_{2} \frac{p_{31}}{2}\right) . \quad \text { (C. } 9\right)
\end{aligned}
$$

\section{- $C H H$ vertex}

$$
\begin{aligned}
& A m p\left(C_{1}^{(0)}, H_{2}^{(-1)}, H_{3}^{(-1)}\right)= \frac{d_{a b c}}{\left(2 \alpha^{\prime}\right)^{3 / 2}}\left\langle c C_{1 \mu \nu \rho}\left(i \partial X^{\mu}+p \psi \psi^{\mu}\right) \psi^{\nu} \psi^{\rho} e^{i p_{1} X}\left(z_{1}\right)\right. \\
&\left.c e^{-\varphi} i \partial X H_{2} \psi e^{i p_{2} X}\left(z_{2}\right) c e^{-\varphi} i \partial X H_{3} \psi e^{i p_{3} X}\left(z_{3}\right)\right\rangle \\
&= \sqrt{2 \alpha^{\prime}} d_{a b c}\left(2 \frac{p_{23}^{\mu}}{2} C_{1 \mu \nu \rho} H_{2 \sigma}^{\nu} H_{3}^{\rho \sigma}+2 C_{1 \mu \nu \rho} H_{3}^{\mu \nu} H_{2 \sigma}^{\rho} \frac{p_{31}^{\sigma}}{2}+2 C_{1 \mu \nu \rho} H_{2 \mu \rho} H_{3 \sigma}^{\nu} \frac{p_{12}^{\sigma}}{2}\right. \\
&\left.+4 \alpha^{\prime} \frac{p_{23}}{2} C_{1 \mu \nu \rho} H_{2 \sigma}^{\nu} H_{3 \lambda}^{\rho} \frac{p_{31}^{\sigma}}{2} \frac{p_{12}^{\lambda}}{2}\right) .
\end{aligned}
$$

\section{Open superstring four-point amplitudes}

Let us discuss the derivation of the 4-point amplitudes with one massive external state, i.e. $\mathcal{A}\left(A_{1}, A_{2}, A_{3}, H_{4}\right)$ and $\mathcal{A}\left(A_{1}, A_{2}, A_{3}, C_{4}\right)$.

\section{D.1 $\mathcal{A}_{A A A C}$ amplitude}

With a judicious choice of super-ghost pictures and c-ghost insertion one has

$$
\begin{aligned}
& \mathcal{A}\left(A_{1}^{(-1)}, A_{2}^{(0)}, A_{3}^{(0)}, C_{4}^{(-1)}\right)=\lim _{\left(z_{1}, z_{2}, z_{4}\right) \rightarrow(\infty, 1,0)} \int_{0}^{1} d z_{3} \\
& \left\langle c e^{-\varphi} a_{1} \psi e^{i k_{1} X}\left(z_{1}\right) c\left(a_{2} \partial X+i k_{2} \psi a_{2} \psi\right) e^{i k_{2} X}\left(z_{2}\right)\left(a_{3} \partial X+i k_{3} \psi a_{3} \psi\right) e^{i k_{3} X}\left(z_{3}\right) c C_{4} \psi \psi \psi e^{i p_{4} X}\left(z_{4}\right)\right\rangle .
\end{aligned}
$$


There are only two kinds of non-vanishing contractions: $\langle\psi(1): \psi \psi:(2) \partial X(3): \psi \psi \psi:(4)\rangle$ $+(2 \leftrightarrow 3)$ and $\langle\psi(1): \psi \psi:(2): \psi \psi:(3): \psi \psi \psi:(4)\rangle$. The first kind of contractions yields

$$
\frac{1}{2 z_{1}}\left[a_{1} \cdot C_{4}: f_{2} a_{3} \cdot P_{3}(x)+\frac{1}{x^{2}} a_{1} \cdot C_{4}: f_{3} a_{2} \cdot P_{2}(x)\right],
$$

where setting $z_{3}=x$ we also have

$$
P_{3}(x)=-\frac{1}{x}\left(k_{1}+\frac{k_{2}}{1-x}\right) \quad P_{2}(x)=\frac{x}{1-x} k_{3}-k_{1} .
$$

It is convenient to define also

$$
P_{4}(x)=-\left(k_{2}+\frac{1}{x} k_{3}\right) \quad P_{1}(x)=k_{2}+x k_{3} .
$$

The second kind of contractions yields

$$
-\frac{1}{2 z_{1}}\left(\frac{a_{1} \cdot f_{2} \cdot C_{4}: f_{3}}{x^{2}}+\frac{a_{1} \cdot f_{3} \cdot C_{4}: f_{2}}{x}+2 \frac{a_{1} \cdot \dot{C}_{4} \cdot \overline{f_{3}} \cdot \dot{f}_{2}}{x(1-x)}\right)
$$

in a self-explanatory index-free notation. Including the momentum factor and the (super)ghost correlators yields (barring $\delta(\Sigma p)$ etc)

$$
\begin{aligned}
& \mathcal{A}\left(A_{1}^{(-1)}, A_{2}^{(0)}, A_{3}^{(0)}, C_{4}^{(-1)}\right)=\int_{0}^{1} d x x^{-\alpha^{\prime} s+1}(1-x)^{-\alpha^{\prime} t}\left[-a_{1} \cdot C_{4}: f_{2}\left(a_{3} \cdot k_{1}+\frac{a_{3} \cdot k_{2}}{1-x}\right)\right. \\
& \left.\quad+a_{1} \cdot C_{4}: f_{3}\left(\frac{a_{2} \cdot k_{3}}{1-x}-\frac{a_{2} \cdot k_{1}}{x}\right)+\frac{a_{1} \cdot f_{2} \cdot C_{4}: f_{3}}{x}+a_{1} \cdot f_{3} \cdot C_{4}: f_{2}+2 \frac{a_{1} \cdot \dot{C}_{4} \cdot \overline{f_{3}} \cdot \dot{f}_{2}}{1-x}\right] .
\end{aligned}
$$

Perusing the factorial properties of $\Gamma(z)$, finally yields

$$
\begin{aligned}
\mathcal{A}_{A A A C}= & \mathcal{B}(1,1)\left\{-a_{1} \cdot C_{4}: f_{2}\left[a_{3} \cdot k_{1}-\frac{u}{t} a_{3} \cdot k_{2}\right]-a_{1} \cdot C_{4}: f_{3}\left[\frac{u}{t} a_{2} \cdot k_{3}-\frac{u}{s} a_{2} \cdot k_{1}\right]\right. \\
& \left.+\frac{u}{s} a_{1} \cdot f_{2} \cdot C_{4}: f_{3}-a_{1} \cdot f_{3} \cdot C_{4}: f_{2}-2 \frac{u}{t} a_{1} \cdot \dot{C}_{4} \cdot \overline{f_{3}} \cdot \dot{f}_{2}\right\} .
\end{aligned}
$$

\section{D.2 $\mathcal{A}_{A A A H}$ amplitude}

We can now embark for a long journey through the computation of $\mathcal{A}_{A A A H}$. With a judicious choice of super-ghost pictures and c-ghost insertions one has

$$
\begin{aligned}
& \mathcal{A}\left(A_{1}^{(-1)}, A_{2}^{(0)}, A_{3}^{(0)}, H_{4}^{(-1)}\right) \\
&=\lim _{\left(z_{1}, z_{2}, z_{4}\right) \rightarrow(\infty, 1,0)} \int_{0}^{1} d z_{3}\left\langle c e^{-\varphi} a_{1} \psi e^{i k_{1} X}\left(z_{1}\right) c\left(a_{2} \partial X+i k_{2} \psi a_{2} \psi\right) e^{i k_{2} X}\left(z_{2}\right)\right. \\
&\left.\quad\left(a_{3} \partial X+i k_{3} \psi a_{3} \psi\right) e^{i k_{3} X}\left(z_{3}\right) c \partial X \cdot H_{4} \cdot \psi e^{i p_{4} X}\left(z_{4}\right)\right\rangle .
\end{aligned}
$$

Since $\langle\psi(1): \psi \psi \psi:(4)\rangle=0$, there are only three kinds of contractions:

$$
\begin{aligned}
& \langle\psi(1) \partial X(3) \partial X(3) \psi \partial X(4)\rangle, \\
& \langle\psi(1): \psi \psi:(2) \partial X(3) \psi \partial X(4)\rangle+(2 \leftrightarrow 3), \\
& \langle\psi(1): \psi \psi:(2): \psi \psi:(3): \psi \partial X(4)\rangle .
\end{aligned}
$$


Exploiting the $P_{i}(x)$ allows to identify 11 terms:

$$
\begin{gathered}
-a_{1} \cdot H_{4} \cdot a_{2} a_{3} \cdot\left(\frac{1}{x} k_{1}+\frac{1}{x(1-x)} k_{2}\right) \rightarrow-a_{1} \cdot H_{4} \cdot a_{2}\left[a_{3} \cdot k_{1} \mathcal{B}(1,1)+a_{3} \cdot k_{2} \mathcal{B}(1,0)\right] \\
\frac{1}{x^{2}} a_{1} \cdot H_{4} \cdot a_{3} a_{2} \cdot\left(\frac{x}{1-x} k_{3}-k_{1}\right) \rightarrow a_{1} \cdot H_{4} \cdot a_{3}\left[a_{2} \cdot k_{3} \mathcal{B}(1,1)-a_{2} \cdot k_{1} \mathcal{B}(0,1)\right. \\
-\frac{1}{(1-x)^{2}} a_{2} \cdot a_{3} a_{1} \cdot H_{4} \cdot\left(k_{2}+\frac{1}{x} k_{3}\right) \rightarrow-a_{2} \cdot a_{3} a_{1} \cdot H_{4} \cdot\left[k_{2} \mathcal{B}(2,-1)+k_{3} \mathcal{B}(1,-1)\right] \\
\frac{1}{x} a_{1} \cdot f_{3} \cdot H_{4} \cdot a_{2} \rightarrow a_{1} \cdot f_{3} \cdot H_{4} \cdot a_{2} \mathcal{B}(1,1) \\
\quad \frac{1}{x^{2}} a_{1} \cdot f_{2} \cdot H_{4} \cdot a_{3} \rightarrow a_{1} \cdot f_{2} \cdot H_{4} \cdot a_{3} \mathcal{B}(0,1) \\
a_{1} \cdot H_{4} \cdot\left(k_{2}+\frac{1}{x} k_{3}\right) a_{2} \cdot\left(\frac{x}{1-x} k_{3}-k_{1}\right) a_{3} \cdot\left(\frac{1}{x} k_{1}+\frac{1}{x(1-x)} k_{2}\right) \rightarrow \\
a_{1} \cdot H_{4} \cdot k_{2}\left\{a_{2} \cdot k_{3}\left[a_{3} \cdot k_{1} \mathcal{B}(2,0)+a_{3} \cdot k_{2} \mathcal{B}(2,-1)\right]-a_{2} \cdot k_{1}\left[a_{3} \cdot k_{1} \mathcal{B}(1,1)+a_{3} \cdot k_{2} \mathcal{B}(1,0)\right]\right\} \\
+a_{1} \cdot H_{4} \cdot k_{3}\left\{a_{2} \cdot k_{3}\left[a_{3} \cdot k_{1} \mathcal{B}(1,0)+a_{3} \cdot k_{2} \mathcal{B}(1,-1)\right]-a_{2} \cdot k_{1}\left[a_{3} \cdot k_{1} \mathcal{B}(0,1)+a_{3} \cdot k_{2} \mathcal{B}(0,0)\right]\right\} \\
-\frac{1}{x} a_{1} \cdot f_{3} \cdot H_{4} \cdot\left(k_{2}+\frac{1}{x} k_{3}\right) a_{2} \cdot\left(\frac{x}{1-x} k_{3}-k_{1}\right) \rightarrow \\
-a_{1} \cdot f_{3} \cdot H_{4} \cdot k_{2}\left[a_{2} \cdot k_{3} \mathcal{B}(2,0)-a_{2} \cdot k_{1} \mathcal{B}(1,1)\right]-a_{1} \cdot f_{3} \cdot H_{4} \cdot k_{3}\left[a_{2} \cdot k_{3} \mathcal{B}(1,0)-a_{2} \cdot k_{1} \mathcal{B}(0,1)\right] \\
a_{1} \cdot f_{2} \cdot H_{4} \cdot\left(k_{2}+\frac{1}{x} k_{3}\right) a_{3}\left(\frac{1}{x} k_{1}+\frac{1}{x(1-x)} k_{2}\right) \rightarrow \\
-\frac{1}{(1-x)^{2}} f_{2}: f_{3} a_{1} \cdot H_{4} \cdot\left(k_{2}+\frac{1}{x} k_{3}\right) \rightarrow-f_{2}: f_{3}\left[a_{1} \cdot H_{4} \cdot k_{2} \mathcal{B}(2,-1)+a_{1} \cdot H_{4} \cdot k_{3} \mathcal{B}(1,-1)\right. \\
a_{1} \cdot f_{2} \cdot H_{4} \cdot k_{2}\left[a_{3} \cdot k_{1} \mathcal{B}(1,1)+a_{3} \cdot k_{2} \mathcal{B}(1,0)\right]+a_{1} \cdot f_{2} \cdot H_{4} \cdot k_{3}\left[a_{3} \cdot k_{1} \mathcal{B}(0,1)+a_{3} \cdot k_{2} \mathcal{B}(0,0)\right] \\
\frac{1}{1-x} a_{1} \cdot f_{3} \cdot f_{2} \cdot H_{4} \cdot\left(k_{2}+\frac{1}{x} k_{3}\right) \rightarrow a_{1} \cdot f_{3} \cdot f_{2} \cdot H_{4} \cdot k_{2} \mathcal{B}(2,0)+a_{1} \cdot f_{3} \cdot f_{2} \cdot H_{4} \cdot k_{3} \mathcal{B}(1,0)
\end{gathered}
$$

Factoring out

$$
\mathcal{B}(1,1)=\frac{\Gamma\left(1-\alpha^{\prime} s\right) \Gamma\left(1-\alpha^{\prime} t\right)}{\Gamma\left(1+\alpha^{\prime} u\right)}
$$

finally yields

$$
\begin{aligned}
& \mathcal{A}_{A A A H}=\mathcal{B}(1,1)\left\{-\frac{u}{t} a_{2} a_{3}\left[\left(1-\alpha^{\prime} s\right) a_{1} H_{4} k_{2}+\left(\alpha^{\prime} u-1\right) a_{1} H_{4} k_{3}\right]-a_{1} H_{4} a_{2}\left(a_{3} k_{1}-a_{3} k_{2} \frac{u}{t}\right)\right. \\
& -a_{1} H_{4} a_{3}\left(a_{2} k_{3} \frac{u}{t}-a_{2} k_{1} \frac{u}{s}\right)+2 \alpha^{\prime} a_{1} H_{4} k_{2}\left[-a_{2} k_{3} a_{3} k_{1} \frac{1-\alpha^{\prime} s}{\alpha^{\prime} t}-a_{2} k_{1}\left(a_{3} k_{1}-a_{3} k_{2} \frac{u}{t}\right)\right] \\
& +2 \alpha^{\prime} a_{1} H_{4} k_{3}\left[-a_{2} k_{3} a_{3} k_{1} \frac{u}{t}-a_{2} k_{1}\left(-a_{3} k_{1} \frac{u}{s}+a_{3} k_{2} \frac{u\left(\alpha^{\prime} u-1\right)}{\alpha^{\prime} s t}\right)\right]+a_{1} f_{2} H_{4} a_{3} \frac{u}{s}-a_{1} f_{3} H_{4} a_{2}
\end{aligned}
$$




$$
\begin{aligned}
& -2 \alpha^{\prime} a_{1} f_{2} H_{4} k_{2}\left(a_{3} k_{1}-a_{3} k_{2} \frac{u}{t}\right)-2 \alpha^{\prime} a_{1} f_{2} H_{4} k_{3}\left(-a_{3} k_{1} \frac{u}{s}+a_{3} k_{2} \frac{u\left(\alpha^{\prime} u-1\right)}{\alpha^{\prime} s t}\right) \\
& +2 \alpha^{\prime} a_{1} f_{3} H_{4} k_{2}\left(-a_{2} k_{3} \frac{1-\alpha^{\prime} s}{\alpha^{\prime} t}-a_{2} k_{1}\right)+2 \alpha^{\prime} a_{1} f_{3} H_{4} k_{3}\left(-a_{2} k_{3} \frac{u}{t}+a_{2} k_{1} \frac{u}{s}\right) \\
& \left.+2 \alpha^{\prime} a_{1} f_{2} f_{3} H_{4} k_{2} \frac{u}{t}-2 \alpha^{\prime} a_{1} f_{2} f_{3} H_{4} k_{3} \frac{u\left(\alpha^{\prime} u-1\right)}{\alpha^{\prime} s t}-2 \alpha^{\prime} a_{1} f_{3} f_{2} H_{4} k_{2} \frac{1-\alpha^{\prime} s}{\alpha^{\prime} t}-2 \alpha^{\prime} a_{1} f_{3} f_{2} H_{4} k_{3} \frac{u}{t}\right\} .
\end{aligned}
$$

\section{D.3 $\mathcal{A}_{A A A C}$ in 4-dimensions}

Let us first consider $\mathcal{A}\left(1^{+} 2^{+} 3^{+} C\right)$.

$$
\begin{aligned}
& \mathcal{A}\left(1^{+} 2^{+} 3^{+} C\right)=i \mathcal{B}(1,1)\left\{a_{1}^{+} f_{2}^{+} p_{4}\left(a_{3}^{+} k_{1}-\frac{u}{t} a_{3}^{+} k_{2}\right)+a_{1}^{+} f_{3}^{+} p_{4}\left(a_{2}^{+} k_{3} \frac{u}{t}-a_{2}^{+} k_{1} \frac{u}{s}\right)\right. \\
& \left.+\frac{u}{s} a_{1}^{+} k_{2} a_{2}^{+} f_{3}^{+} p_{4}+a_{1}^{+} k_{3} a_{3}^{+} f_{2}^{+} p_{4}-\left[a_{2}^{+} k_{3}\left(a_{3}^{+} f_{1}^{+} p_{4}-a_{1}^{+} f_{3}^{+} p_{4}\right)-a_{3}^{+} k_{2}\left(a_{2}^{+} f_{1}^{+} p_{4}-a_{1}^{+} f_{2}^{+} p_{4}\right)\right]\right\} .
\end{aligned}
$$

The final result is

$$
\mathcal{A}\left(1^{+} 2^{+} 3^{+} C\right)=i \mathcal{B}(1,1) m_{C}^{4} \frac{[13]}{\langle 12\rangle\langle 23\rangle} .
$$

Let us now consider $\mathcal{A}\left(1^{-} 2^{-} 3^{+} C\right)$. Choosing

$$
a_{1}^{-\mu}=\frac{\left\langle 1\left|\sigma^{\mu}\right| 2\right]}{[21]} \quad a_{2}^{-\mu}=\frac{\left\langle 2\left|\sigma^{\mu}\right| 1\right]}{[12]} \quad a_{3}^{+\mu}=\frac{\left\langle 1\left|\sigma^{\mu}\right| 3\right]}{\langle 13\rangle},
$$

the amplitude simplifies as follows

$$
\begin{aligned}
\mathcal{A}\left(1^{-} 2^{-} 3^{+} C\right) & =i\left(-a_{1}^{-} \cdot C: f_{3}^{+} a_{2}^{-} k_{3} \frac{u}{t}-a_{1}^{-} f_{3}^{+} \cdot C: f_{2}^{-}-2 \frac{u}{t} a_{1}^{-} \cdot \dot{C} \cdot \overline{f_{3}^{+}} \cdot \dot{f}_{2}^{-}\right) \mathcal{B}(1,1) \\
& =i a_{1}^{-} k_{3} a_{2}^{-} k_{3} a_{3}^{+} k_{2} \mathcal{B}(1,1)=i \frac{[13]}{\langle 12\rangle}\langle 23\rangle^{3} \mathcal{B}(1,1) .
\end{aligned}
$$

\section{D.4 $\mathcal{A}_{A A A H}$ in 4-dimensions}

Expressing the 4-momentum of a $H$ massive spin 2 state as $p=k_{1}+k_{2}$, with $k_{1}^{2}=k_{2}^{2}=0$ and $2 k_{1} \cdot k_{2}=p^{2}=-1 / \alpha^{\prime}$, it is possible to write its physical polarizations in the spinor helicity formalism. If we define $k_{1 \alpha \dot{\alpha}}=u_{\alpha} \bar{u}_{\dot{\alpha}}$ and $k_{2 \alpha \dot{\alpha}}=v_{\alpha} \bar{v}_{\dot{\alpha}}$, we have

$$
\begin{aligned}
H_{\alpha \dot{\alpha} \beta \dot{\beta}}= & c_{0}\left(u_{\alpha} u_{\beta} \bar{u}_{\dot{\alpha}} \bar{u}_{\dot{\beta}}+v_{\alpha} v_{\beta} \bar{v}_{\dot{\alpha}} \bar{v}_{\dot{\beta}}-\left(u_{\alpha} v_{\beta}+u_{\beta} v_{\alpha}\right)\left(\bar{u}_{\dot{\alpha}} \bar{v}_{\dot{\beta}}+\bar{u}_{\dot{\alpha}} \bar{v}_{\dot{\beta}}\right)\right) \\
& +c_{1}\left(u_{\alpha} u_{\beta}\left(\bar{u}_{\dot{\alpha}} \bar{v}_{\dot{\beta}}+\bar{u}_{\dot{\beta}} \bar{v}_{\dot{\alpha}}\right)-\bar{v}_{\dot{\alpha}} \bar{v}_{\dot{\beta}}\left(u_{\alpha} v_{\beta}+u_{\beta} v_{\alpha}\right)\right) \\
& +c_{-1}\left(v_{\alpha} v_{\beta}\left(\bar{u}_{\dot{\alpha}} \bar{v}_{\dot{\beta}}+\bar{u}_{\dot{\beta}} \bar{v}_{\dot{\alpha}}\right)-\bar{u}_{\dot{\alpha}} \bar{u}_{\dot{\beta}}\left(u_{\alpha} v_{\beta}+u_{\beta} v_{\alpha}\right)\right) \\
& +c_{2} u_{\alpha} u_{\beta} \bar{v}_{\dot{\alpha}} \bar{v}_{\dot{\beta}}+c_{-2} v_{\alpha} v_{\beta} \bar{u}_{\dot{\alpha}} \bar{u}_{\dot{\alpha}} .
\end{aligned}
$$

Recalling that $\mathcal{A}\left(A_{1}, A_{2}, H\right) \propto \operatorname{tr}\left(f_{1} H f_{2}\right)$, we can express the coupling between two vector bosons and each helicity component of $H$. As shown in table 1, $H$ couples only to vector bosons with opposite helicity. 


\begin{tabular}{|c||c|c|c|c|}
\hline & $f_{1}^{-} f_{2}^{+}$ & $f_{1}^{-} f_{2}^{-}$ & $f_{1}^{+} f_{2}^{+}$ & $f_{1}^{+} f_{2}^{-}$ \\
\hline \hline$c_{0}$ & $\langle u 1\rangle^{2}[u 2]^{2}+\langle v 1\rangle^{2}[v 2]^{2}$ & 0 & 0 & $\langle u 2\rangle^{2}[u 1]^{2}+\langle v 2\rangle^{2}[v 1]^{2}$ \\
& $-4\langle u 1\rangle\langle v 1\rangle[u 2][v 2]$ & & & $-4\langle u 2\rangle\langle v 2\rangle[u 1][v 1]$ \\
\hline$c_{1}$ & $2\langle u 1\rangle^{2}[u 2][v 2]-2\langle u 1\rangle\langle v 1\rangle[v 2]^{2}$ & 0 & 0 & $2\langle u 2\rangle^{2}[u 1][v 1]-2\langle u 2\rangle\langle v 1\rangle[v 1]^{2}$ \\
\hline$c_{-1}$ & $2\langle v 1\rangle^{2}[v 2][u 2]-2\langle v 1\rangle\langle u 1\rangle[u 2]^{2}$ & 0 & 0 & $2\langle v 2\rangle^{2}[v 1][u 1]-2\langle v 2\rangle\langle u 1\rangle[u 1]^{2}$ \\
\hline$c_{2}$ & $\langle u 1\rangle^{2}[v 2]^{2}$ & 0 & 0 & $\langle u 2\rangle^{2}[v 1]^{2}$ \\
\hline$c_{-2}$ & $\langle v 1\rangle^{2}[u 2]^{2}$ & 0 & 0 & $\langle v 2\rangle^{2}[u 1]^{2}$ \\
\hline
\end{tabular}

Table 1. In the table we list all couplings between a spin-2 massive state $H$ and two vector bosons $A_{1}, A_{2}$. The momentum of $H$ is $p_{\alpha \dot{\alpha}}=u_{\alpha} \bar{u}_{\dot{\alpha}}+v_{\alpha} \bar{v}_{\dot{\alpha}}$. It is worth to notice that $H$ couples only to couple of vector bosons with opposite helicities.

Let us discuss the case in which only the scalar component of $H$ (the trace of $H$ in 4 dimensions) couples to the three vector bosons and let us start with the amplitude $\mathcal{A}\left(1^{+} 2^{+} 3^{+} H_{0}\right)$. Choosing

$$
a_{1}^{+}=\frac{\mid 1]\langle 2|}{\langle 21\rangle} \quad a_{2}^{+}=\frac{\mid 2]\langle 1|}{\langle 12\rangle} \quad a_{3}^{+}=\frac{\mid 3]\langle 1|}{\langle 13\rangle}
$$

some of the scalar products appearing in eq. (D.25) vanish: $a_{2}^{+} \cdot a_{3}^{+}=0, a_{1}^{+} \cdot k_{2}=0, a_{2}^{+} \cdot k_{1}=0$ and $a_{3}^{+} \cdot k_{1}=0$, and the amplitude simplifies significantly

$$
\begin{aligned}
& \mathcal{A}\left(1^{+} 2^{+} 3^{+} H_{0}\right)=a_{1} H_{0} a_{2} a_{3} k_{2} \frac{u}{t}-a_{1} H_{0} a_{3} a_{2} k_{3} \frac{u}{t}+a_{1} f_{2} H_{0} a_{3} \frac{u}{s}-a_{1} f_{3} H_{0} a_{2} \\
& +2 \alpha^{\prime} a_{1} f_{2} H_{0} k_{2} a_{3} k_{2} \frac{u}{t}+2 \alpha^{\prime} a_{1} f_{2} H_{0} k_{3} a_{3} k_{2} \frac{u(s+t)}{s t} \\
& -2 \alpha^{\prime} a_{1} f_{3} H_{0} k_{2} a_{2} k_{3} \frac{u+t}{t}-2 \alpha^{\prime} a_{1} f_{3} H_{0} k_{3} a_{2} k_{3} \frac{u}{t} \\
& +2 \alpha^{\prime} a_{1} f_{2} f_{3} H_{0} k_{2} \frac{u}{t}+2 \alpha^{\prime} a_{1} f_{2} f_{3} H_{0} k_{3} \frac{u(s+t)}{s t}-2 \alpha^{\prime} a_{1} f_{3} f_{2} H_{0} k_{2} \frac{u+t}{t}-2 \alpha^{\prime} a_{1} f_{3} f_{2} H_{0} k_{3} \frac{u}{t} .
\end{aligned}
$$

The scalar 4-dimensional polarization of $H_{0}$ is $H_{0 \mu \nu}=\eta_{\mu \nu}+\alpha^{\prime} p_{\mu} p_{\nu}$. Let us consider the diagonal part of the polarization of $H_{0}$.

$$
\begin{aligned}
a_{1} a_{2} a_{3} k_{2} \frac{u}{t}-a_{1} a_{3} a_{2} k_{3} \frac{u}{t} & =2(s+t) \frac{[13]}{\langle 12\rangle\langle 23\rangle} \\
a_{1} f_{2} a_{3} \frac{u}{s}-a_{1} f_{3} a_{2} & =4 t \frac{[13]}{\langle 12\rangle\langle 23\rangle} \\
2 \alpha^{\prime} a_{1} f_{2} k_{3} a_{3} k_{2} \frac{u(s+t)}{s t} & =-2 \alpha^{\prime} t(s+t) \frac{[13]}{\langle 12\rangle\langle 23\rangle} \\
-2 \alpha^{\prime} a_{1} f_{3} f_{2} k_{3} \frac{u}{t} & =-2 \alpha^{\prime} u t \frac{[13]}{\langle 12\rangle\langle 23\rangle}
\end{aligned}
$$


Let us now consider the longitudinal part of the polarization: $\alpha^{\prime} p_{\mu} p_{\nu}$.

$$
\begin{aligned}
\alpha^{\prime} a_{1} p a_{2} p a_{3} k_{2} \frac{u}{t}-\alpha^{\prime} a_{1} p a_{3} p a_{2} k_{3} \frac{u}{t}=0 \\
\alpha^{\prime} a_{1} f_{2} p a_{3} p \frac{u}{s}-\alpha^{\prime} a_{1} f_{3} p a_{2} p=-t \frac{[13]}{\langle 12\rangle\langle 23\rangle} \\
2 \alpha^{\prime 2} a_{1} f_{2} p p k_{2} a_{3} k_{2} \frac{u}{t}+2 \alpha^{\prime 2} a_{1} f_{2} p p k_{3} a_{3} k_{2} \frac{u(s+t)}{s t}=\alpha^{\prime}(s+t)^{2} \frac{[13]}{\langle 12\rangle\langle 23\rangle} \\
-2 \alpha^{\prime 2} a_{1} f_{3} p p k_{2} a_{2} k_{3} \frac{u+t}{t}-2 \alpha^{\prime 2} a_{1} f_{3} p p k_{3} a_{2} k_{3} \frac{u}{t}=\alpha^{\prime} t(u+t) \frac{[13]}{\langle 12\rangle\langle 23\rangle} \\
2 \alpha^{\prime 2} a_{1} f_{2} f_{3} p p k_{2} \frac{u}{t}+2 \alpha^{\prime 2} a_{1} f_{2} f_{3} p p k_{3} \frac{u(s+t)}{s t}=\alpha^{\prime} u(s+t) \frac{[13]}{\langle 12\rangle\langle 23\rangle} \\
-2 \alpha^{\prime 2} a_{1} f_{3} f_{2} p p k_{2} \frac{u+t}{t}-2 \alpha^{\prime 2} a_{1} f_{3} f_{2} p p k_{3} \frac{u}{t}=\alpha^{\prime}(s+t)(u+t) \frac{[13]}{\langle 12\rangle\langle 23\rangle} .
\end{aligned}
$$

Using the identity $s+t+u=1 / \alpha^{\prime}$, the sum of the terms in eqs. (D.33), (D.34) yields

$$
\mathcal{A}\left(1^{+} 2^{+} 3^{+} H_{0}\right) \propto \frac{1}{\alpha^{\prime}} \frac{[13]}{\langle 12\rangle\langle 23\rangle} .
$$

Let us consider the amplitude $\mathcal{A}\left(1^{-} 2^{-} 3^{+} H_{0}\right)$. Choosing

$$
a_{1}^{-}=\frac{\mid 2]\langle 1|}{[21]} \quad a_{2}^{-}=\frac{\mid 1]\langle 2|}{[12]} \quad a_{3}^{+}=\frac{\mid 3]\langle 1|}{\langle 13\rangle},
$$

we can enforce the conditions $a_{i} \cdot a_{j}=0$ and $a_{1} \cdot k_{2}=a_{2} \cdot k_{1}=a_{3} \cdot k_{1}=0$. The resulting amplitude looks like

$$
\begin{aligned}
& \mathcal{A}\left(1^{-} 2^{-} 3^{+} H_{0}\right)=a_{1} H_{0} a_{2} \quad a_{3} k_{2} \frac{u}{t}+a_{1} H_{0} a_{3} a_{2} k_{3} \frac{u}{t}+a_{1} k_{3} a_{3} H_{0} a_{2}+2 \alpha^{\prime} a_{1} k_{3} a_{3} H_{0} k_{2} a_{2} k_{3} \frac{u+t}{t} \\
& +2 \alpha^{\prime} a_{1} k_{3} a_{3} H_{0} k_{3} a_{2} k_{3} \frac{u}{t}-2 \alpha^{\prime} a_{1} k_{3} a_{3} k_{2} a_{2} H_{0} k_{2} \frac{u+t}{t}-2 \alpha^{\prime} a_{1} k_{3} a_{3} k_{2} a_{2} H_{0} k_{3} \frac{u}{t} .
\end{aligned}
$$

The diagonal part of the polarization of $H_{0}$ produces

$$
2 \alpha^{\prime} a_{1} k_{3} a_{3} H_{0} k_{2} a_{2} k_{3} \frac{u+t}{t}-2 \alpha^{\prime} a_{1} k_{3} a_{3} k_{2} a_{2} H_{0} k_{3} \frac{u}{t}=2 \alpha^{\prime} \frac{\left.{ }^{\prime} 13\right]\langle 23\rangle^{3}}{\langle 12\rangle} .
$$

The longitudinal part of the polarization of $H_{0}$ yields

$$
\alpha^{\prime} a_{1} p \quad a_{2} p \quad a_{3} k_{2} \frac{u}{t}+\alpha^{\prime} a_{1} p a_{3} p \quad a_{2} k_{3} \frac{u}{t}+\alpha^{\prime} a_{1} k_{3} a_{3} p a_{2} p=3 \alpha^{\prime} \frac{[13]\langle 23\rangle^{3}}{\langle 12\rangle}
$$

Finally the result is

$$
\mathcal{A}\left(1^{-} 2^{-} 3^{+} H_{0}\right) \propto \alpha^{\prime} \frac{[13]}{\langle 12\rangle}\langle 23\rangle^{3} .
$$

Let us consider the case in which the spin-2 tensor $H$ with helicity $u_{\alpha}(4) u_{\alpha}(4) \bar{v}_{\dot{\alpha}}(5) \bar{v}_{\dot{\alpha}}(5)$, with $p=k_{4}+k_{5}$ the momentum of $H$, having helicity $h=2$ couples to three vector bosons, i.e. $\mathcal{A}\left(1^{-} 2^{+} 3^{+} H\right)$. Choosing the following parametrization for the polarization vectors of the incoming gluons

$$
a_{1}^{-}=\frac{\mid 2]\langle 1|}{[21]}, \quad a_{2}^{+}=\frac{\mid 2]\langle 1|}{\langle 12\rangle}, \quad a_{3}^{+}=\frac{\mid 3]\langle 1|}{\langle 13\rangle},
$$


we have $a_{i} \cdot a_{j}=0$ and $a_{1}^{-} \cdot k_{2}=a_{2}^{+} \cdot k_{1}=a_{3}^{+} \cdot k_{1}=0$.

$$
\begin{aligned}
& \mathcal{A}\left(1^{-} 2^{+} 3^{+} H\right)= \\
& a_{1}^{-} H a_{2}^{+} a_{3}^{+} k_{2} \frac{u}{t}-a_{1}^{-} H a_{3}^{+} a_{2}^{+} k_{3} \frac{u}{t}-a_{1}^{-} f_{3}^{+} H a_{2}^{+}-2 \alpha^{\prime} a_{1}^{-} f_{3}^{+} H k_{2} a_{2}^{+} k_{3} \frac{u+t}{t} \\
& \quad-2 \alpha^{\prime} a_{1}^{-} f_{3}^{+} H k_{3} a_{2}^{+} k_{3} \frac{u}{t}-2 \alpha^{\prime} a_{1}^{-} f_{3}^{+} f_{2}^{+} H k_{2} \frac{u+t}{t}-2 \alpha^{\prime} a_{1}^{-} f_{3}^{+} f_{2}^{+} H k_{3} \frac{u}{t} \\
& =a_{1}^{-} H a_{2}^{+} a_{3}^{+} k_{2} \frac{u}{t}-a_{1}^{-} H a_{3}^{+} a_{2}^{+} k_{3} \frac{u}{t}+a_{1}^{-} k_{3} a_{3}^{+} H a_{2}^{+}+2 \alpha^{\prime} a_{1}^{-} k_{3} a_{3}^{+} H k_{2} a_{2}^{+} k_{3} \frac{u+t}{t} \\
& \quad+2 \alpha^{\prime} a_{1}^{-} k_{3} a_{3}^{+} H k_{3} a_{2}^{+} k_{3} \frac{u}{t}-2 \alpha^{\prime} a_{1}^{-} k_{3} a_{3}^{+} k_{2} a_{2}^{+} H k_{2} \frac{u+t}{t}-2 \alpha^{\prime} a_{1}^{-} k_{3} a_{3}^{+} k_{2} a_{2}^{+} H k_{3} \frac{u}{t} .
\end{aligned}
$$

So we have

$$
\begin{aligned}
& a_{1}^{-} H a_{2}^{+} \quad a_{3}^{+} k_{2} \frac{u}{t}-a_{1}^{-} H a_{3}^{+} a_{2}^{+} k_{3} \frac{u}{t}=4 \mathcal{A} \frac{[25][45]\langle 45\rangle^{2}}{[21]\langle 14\rangle} \\
& a_{1}^{-} k_{3} a_{3}^{+} H a_{2}^{+}=4 \mathcal{A} \frac{[23][25][35]\langle 45\rangle^{2}\langle 23\rangle}{[21][13]\langle 14\rangle^{2}} \\
& 2 \alpha^{\prime} a_{1}^{-} k_{3} a_{3}^{+} H k_{2} a_{2}^{+} k_{3} \frac{u+t}{t}+2 \alpha^{\prime} a_{1}^{-} k_{3} a_{3}^{+} H k_{3} a_{2}^{+} k_{3} \frac{u}{t} \\
& =-4 \alpha^{\prime} \mathcal{A} \frac{[23][35]\langle 13\rangle\langle 45\rangle^{2}}{[21][13]\langle 14\rangle^{3}}(t[25]\langle 24\rangle-u[15]\langle 14\rangle) \\
& \quad-2 \alpha^{\prime} a_{1}^{-} k_{3} a_{3}^{+} k_{2} a_{2}^{+} H k_{2} \frac{u+t}{t}-2 \alpha^{\prime} a_{1}^{-} k_{3} a_{3}^{+} k_{2} a_{2}^{+} H k_{3} \frac{u}{t} \\
& =-4 \alpha^{\prime} \mathcal{A} \frac{[23][25]\langle 12\rangle\langle 45\rangle^{2}}{[21][13]\langle 14\rangle^{3}}(t[25]\langle 24\rangle-u[15]\langle 14\rangle),
\end{aligned}
$$

where

$$
\mathcal{A}=\frac{\langle 14\rangle^{4}[13]}{\langle 12\rangle\langle 23\rangle\langle 45\rangle^{2}} .
$$

The sum of the terms in eq. (D.43), produces the amplitude

$$
\mathcal{A}\left(1^{-} 2^{+} 3^{+} H\right) \propto \frac{1}{\alpha^{\prime}} \mathcal{A}=\frac{1}{\alpha^{\prime}} \frac{\langle 14\rangle^{4}[13]}{\langle 12\rangle\langle 23\rangle\langle 45\rangle^{2}} .
$$

Open Access. This article is distributed under the terms of the Creative Commons Attribution License (CC-BY 4.0), which permits any use, distribution and reproduction in any medium, provided the original author(s) and source are credited.

\section{References}

[1] H. Bondi, M.G.J. van der Burg and A.W.K. Metzner, Gravitational waves in general relativity. \%. Waves from axisymmetric isolated systems, Proc. Roy. Soc. Lond. A 269 (1962) 21 [INSPIRE].

[2] G. Barnich and C. Troessaert, Symmetries of asymptotically flat 4 dimensional spacetimes at null infinity revisited, Phys. Rev. Lett. 105 (2010) 111103 [arXiv:0909.2617] [INSPIRE].

[3] G. Barnich and C. Troessaert, Supertranslations call for superrotations, PoS (2010) 010 [arXiv:1102.4632] [INSPIRE]. 
[4] G. Barnich and C. Troessaert, BMS charge algebra, JHEP 12 (2011) 105 [arXiv:1106. 0213] [INSPIRE].

[5] A. Strominger, On BMS Invariance of Gravitational Scattering, JHEP 07 (2014) 152 [arXiv: 1312.2229] [INSPIRE].

[6] T. He, V. Lysov, P. Mitra and A. Strominger, BMS supertranslations and Weinberg's soft graviton theorem, JHEP 05 (2015) 151 [arXiv: 1401.7026] [INSPIRE].

[7] F.E. Low, Scattering of light of very low frequency by systems of spin 1/2, Phys. Rev. 96 (1954) 1428 [inSPIRE].

[8] S. Weinberg, Infrared photons and gravitons, Phys. Rev. 140 (1965) B516 [INSPIRE].

[9] D.J. Gross and R. Jackiw, Low-Energy Theorem for Graviton Scattering, Phys. Rev. 166 (1968) 1287 [INSPIRE].

[10] F. Cachazo and A. Strominger, Evidence for a New Soft Graviton Theorem, arXiv: 1404.4091 [NSPIRE].

[11] Z. Bern, S. Davies and J. Nohle, On Loop Corrections to Subleading Soft Behavior of Gluons and Gravitons, Phys. Rev. D 90 (2014) 085015 [arXiv: 1405.1015] [INSPIRE].

[12] S. He, Y.-t. Huang and C. Wen, Loop Corrections to Soft Theorems in Gauge Theories and Gravity, JHEP 12 (2014) 115 [arXiv:1405.1410] [INSPIRE].

[13] F. Cachazo and E.Y. Yuan, Are Soft Theorems Renormalized?, arXiv:1405.3413 [InSPIRE].

[14] M. Bianchi, S. He, Y.-t. Huang and C. Wen, More on Soft Theorems: Trees, Loops and Strings, arXiv: 1406.5155 [INSPIRE].

[15] A.J. Larkoski, D. Neill and I.W. Stewart, Soft Theorems from Effective Field Theory, JHEP 06 (2015) 077 [arXiv:1412.3108] [INSPIRE].

[16] H. Elvang and Y.-t. Huang, Scattering Amplitudes, arXiv:1308.1697 [INSPIRE].

[17] E. Casali, Soft sub-leading divergences in Yang-Mills amplitudes, JHEP 08 (2014) 077 [arXiv: 1404.5551] [INSPIRE].

[18] B.U.W. Schwab and A. Volovich, Subleading Soft Theorem in Arbitrary Dimensions from Scattering Equations, Phys. Rev. Lett. 113 (2014) 101601 [arXiv:1404.7749] [INSPIRE].

[19] N. Afkhami-Jeddi, Soft Graviton Theorem in Arbitrary Dimensions, arXiv:1405.3533 [INSPIRE].

[20] T. Adamo, E. Casali and D. Skinner, Perturbative gravity at null infinity, Class. Quant. Grav. 31 (2014) 225008 [arXiv:1405.5122] [INSPIRE].

[21] B.U.W. Schwab, Subleading Soft Factor for String Disk Amplitudes, JHEP 08 (2014) 062 [arXiv: 1406.4172] [INSPIRE].

[22] Z. Bern, S. Davies, P. Di Vecchia and J. Nohle, Low-Energy Behavior of Gluons and Gravitons from Gauge Invariance, Phys. Rev. D 90 (2014) 084035 [arXiv:1406.6987] [INSPIRE].

[23] M. Bianchi and L. Lopez, Pair Production of small Black Holes in Heterotic String Theories, JHEP 07 (2010) 065 [arXiv: 1002.3058] [INSPIRE].

[24] M. Bianchi, L. Lopez and R. Richter, On stable higher spin states in Heterotic String Theories, JHEP 03 (2011) 051 [arXiv: 1010.1177] [INSPIRE]. 
[25] M. Bianchi and P. Teresi, Scattering higher spins off D-branes, JHEP 01 (2012) 161 [arXiv: 1108.1071] [INSPIRE].

[26] W. Black and C. Monni, High energy string-brane scattering for massive states, Nucl. Phys. B 859 (2012) 299 [arXiv:1107.4321] [INSPIRE].

[27] D.P. Skliros and M.B. Hindmarsh, Covariant Vertex Operators for Cosmic Strings, arXiv:0911.5354 [INSPIRE].

[28] D. Skliros and M. Hindmarsh, String Vertex Operators and Cosmic Strings, Phys. Rev. D 84 (2011) 126001 [arXiv:1107.0730] [INSPIRE].

[29] E. Dudas and J. Mourad, String theory predictions for future accelerators, Nucl. Phys. B 575 (2000) 3 [hep-th/9911019] [INSPIRE].

[30] D. Chialva, R. Iengo and J.G. Russo, Cross sections for production of closed superstrings at high energy colliders in brane world models, Phys. Rev. D 71 (2005) 106009 [hep-ph/0503125] [INSPIRE].

[31] M. Bianchi and A.V. Santini, String predictions for near future colliders from one-loop scattering amplitudes around D-brane worlds, JHEP 12 (2006) 010 [hep-th/0607224] [INSPIRE].

[32] W.-Z. Feng, D. Lüst, O. Schlotterer, S. Stieberger and T.R. Taylor, Direct Production of Lightest Regge Resonances, Nucl. Phys. B 843 (2011) 570 [arXiv:1007.5254] [INSPIRE].

[33] W.-Z. Feng and T.R. Taylor, Higher Level String Resonances in Four Dimensions, Nucl. Phys. B 856 (2012) 247 [arXiv:1110.1087] [InSPIRE].

[34] L.A. Anchordoqui, H. Goldberg, D. Lüst, S. Stieberger and T.R. Taylor, String Phenomenology at the LHC, Mod. Phys. Lett. A 24 (2009) 2481 [arXiv:0909.2216] [INSPIRE].

[35] C.R. Mafra, O. Schlotterer and S. Stieberger, Complete N-Point Superstring Disk Amplitude I. Pure Spinor Computation, Nucl. Phys. B 873 (2013) 419 [arXiv:1106.2645] [InSPIRE].

[36] C.R. Mafra, O. Schlotterer and S. Stieberger, Complete N-Point Superstring Disk Amplitude II. Amplitude and Hypergeometric Function Structure, Nucl. Phys. B 873 (2013) 461 [arXiv:1106.2646] [INSPIRE].

[37] J.E. Paton and H.-M. Chan, Generalized veneziano model with isospin, Nucl. Phys. B 10 (1969) 516 [InSPIRE].

[38] M.L. Mangano and S.J. Parke, Multiparton amplitudes in gauge theories, Phys. Rept. 200 (1991) 301 [hep-th/0509223] [INSPIRE].

[39] I.G. Koh, W. Troost and A. Van Proeyen, Covariant Higher Spin Vertex Operators in the Ramond Sector, Nucl. Phys. B 292 (1987) 201 [INSPIRE].

[40] S. Ferrara and B. Zumino, Transformation Properties of the Supercurrent, Nucl. Phys. B 87 (1975) 207 [inSPIRE].

[41] H. Kawai, D.C. Lewellen and S.H.H. Tye, A Relation Between Tree Amplitudes of Closed and Open Strings, Nucl. Phys. B 269 (1986) 1 [inSPIRE].

[42] L.A. Barreiro and R. Medina, RNS derivation of $N$-point disk amplitudes from the revisited S-matrix approach, Nucl. Phys. B 886 (2014) 870 [arXiv:1310.5942] [INSPIRE].

[43] L.A. Barreiro and R. Medina, Revisiting the S-matrix approach to the open superstring low energy effective lagrangian, JHEP 10 (2012) 108 [arXiv:1208.6066] [INSPIRE]. 
[44] S. Stieberger and T.R. Taylor, Multi-Gluon Scattering in Open Superstring Theory, Phys. Rev. D 74 (2006) 126007 [hep-th/0609175] [INSPIRE].

[45] S. Stieberger and T.R. Taylor, Supersymmetry Relations and MHV Amplitudes in Superstring Theory, Nucl. Phys. B 793 (2008) 83 [arXiv:0708.0574] [INSPIRE].

[46] P. Di Vecchia, R. Marotta and M. Mojaza, Soft theorem for the graviton, dilaton and the Kalb-Ramond field in the bosonic string, JHEP 05 (2015) 137 [arXiv: 1502.05258] [INSPIRE].

[47] M. Zlotnikov, Sub-sub-leading soft-graviton theorem in arbitrary dimension, JHEP 10 (2014) 148 [arXiv: 1407.5936] [InSPIRE].

[48] C. Kalousios and F. Rojas, Next to subleading soft-graviton theorem in arbitrary dimensions, JHEP 01 (2015) 107 [arXiv: 1407.5982] [INSPIRE].

[49] B.U.W. Schwab, A Note on Soft Factors for Closed String Scattering, JHEP 03 (2015) 140 [arXiv: 1411.6661] [INSPIRE].

[50] M. Ademollo et al., Soft Dilations and Scale Renormalization in Dual Theories, Nucl. Phys. B 94 (1975) 221 [inSPIRE].

[51] W.-M. Chen, Y.-t. Huang and C. Wen, From U(1) to E8: soft theorems in supergravity amplitudes, JHEP 03 (2015) 150 [arXiv:1412.1811] [INSPIRE].

[52] M. Bianchi and A.L. Guerrieri, On the soft limit of closed string tree level amplitudes with massive states, in preparation.

[53] S. Stieberger and T.R. Taylor, Graviton as a Pair of Collinear Gauge Bosons, Phys. Lett. B 739 (2014) 457 [arXiv: 1409.4771] [InSPIRE].

[54] S. Stieberger and T.R. Taylor, Graviton Amplitudes from Collinear Limits of Gauge Amplitudes, Phys. Lett. B 744 (2015) 160 [arXiv:1502.00655] [INSPIRE]. 